Field Test of The PNNL Automated Radioxenon Sampler/Analyzer (ARSA)

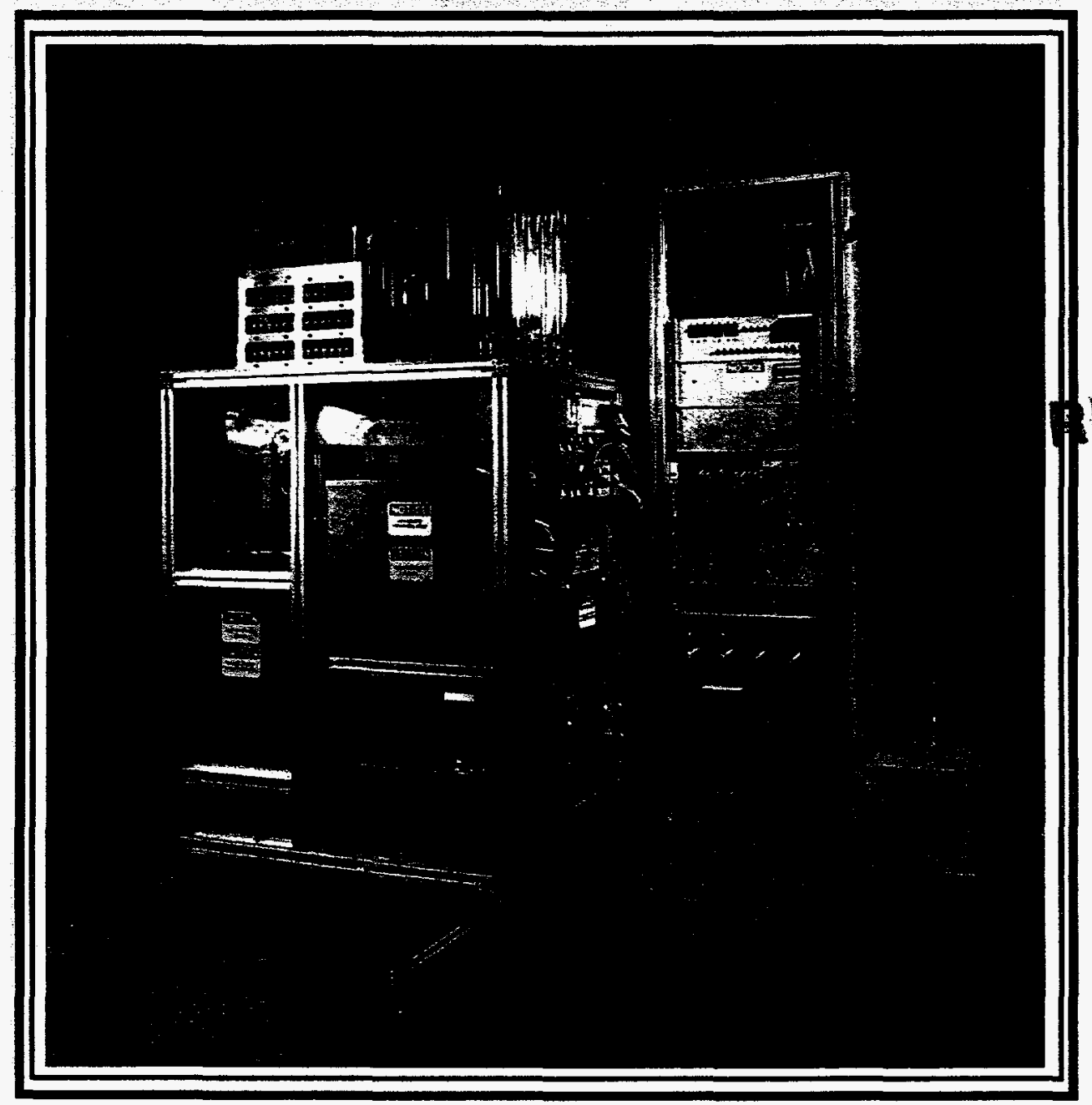

ECEIVED OCT 131998

C.TI

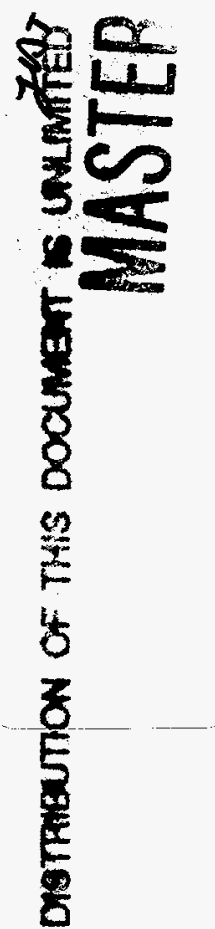

201 Varick Street, 5th Floor, New York, NY 10014-4811 http://www.eml.doe.gov 


\title{
FIELd TEST OF THE PNNL AUTOMATED RADIOXENON SAMPLER/ANALYZER (ARSA)
}

\author{
R. J. Lagomarsino, E. Ku, C. G. Sanderson and N. Latner
}

\author{
Environmental Measurements Laboratory \\ U.S. Department of Energy \\ 201 Varick Street, 5th Floor \\ New York, NY 10014-4811
}

July 1998

Edited by: Air Force Technical Applications Center

\begin{abstract}
DISCLAIMER
"This report was prepared as an account of work sponsored by an agency of the United States Government. Neither the United States Government nor any agency thereof, nor any of their employees, makes any warranty, express or implied, or assumes any legal liability or responsibility for the accuracy, completeness, or usefulness of any information, apparatus, product, or process disclosed, or represents that its use would not infringe privately owned rights. Reference herein to any specific commercial product, process, or service by trade name, trademark, manufacturer, or otherwise, does not necessarily constitute or imply its endorsement, recommendation, or favoring by the United States Government or any agency thereof. The views and opinions of authors expressed herein do not necessarily state or reflect those of the United States Government or any agency thereof."
\end{abstract}

This report has been reproduced directly from the best available copy. 


\section{DISCLAIMER}

Portions of this document may be illegible in electronic image products. Images are produced from the best available original document. 


\section{Anstract}

As part of the requirements of the Comprehensive Test Ban Treaty (CTBT), the Automated Radioxenon/Sampler Analyzer (ARSA) was designed and engineered by the Pacific Northwest National Laboratory (PNNL). The instrument is to provide near real-time detection and measurement of the radioxenons released into the atmosphere after a nuclear test. Forty-six field tests, designed to determine the performance of the ARSA prototype under simulated field conditions, were conducted at EML from March to December 1997. This final report contains detailed results of the tests with recommendations for improvements in instrument performance. 


\section{Alcknowledgment}

The authors thank $1^{\text {st }}$ Lt. Jason R. Price, SSgt. Andrew J. Austin, and SrA Terry M. Jude of TOD for their help and support in conducting the field test and for their contributions to this report. Also, thanks go to Capt. Matthew Murdough, AFTAC/TOD for his input, development, and direction of the field test. 


\section{T able of Contents}

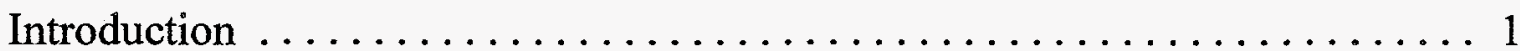

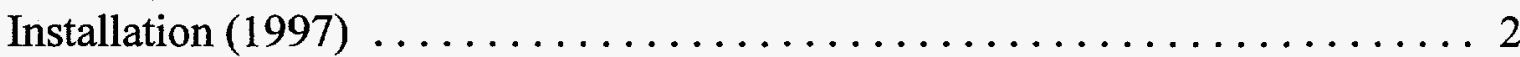

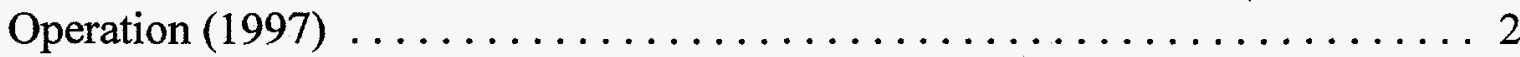

General Test Descriptions $\ldots \ldots \ldots \ldots \ldots \ldots \ldots \ldots \ldots \ldots \ldots \ldots \ldots$

General Theory of Sampler Operation $\ldots \ldots \ldots \ldots \ldots \ldots \ldots \ldots \ldots$

ARSA Improvements Following the Smoke Condition Event $\ldots \ldots \ldots \ldots 6$

Archive Bottle Analysis $\ldots \ldots \ldots \ldots \ldots \ldots \ldots \ldots \ldots \ldots \ldots \ldots$

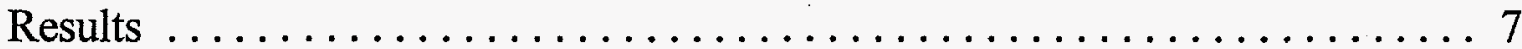

Conclusions and Recommendations $\ldots \ldots \ldots \ldots \ldots \ldots \ldots \ldots \ldots \ldots \ldots$

References $\ldots \ldots \ldots \ldots \ldots \ldots \ldots \ldots \ldots \ldots \ldots \ldots \ldots \ldots \ldots \ldots, 16$

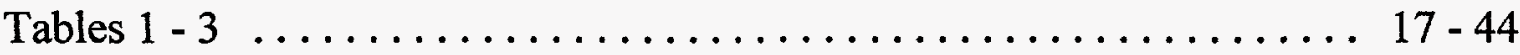

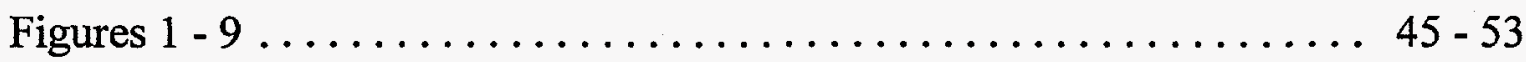

Appendix A. Results of the Individual Field Tests $\ldots \ldots \ldots \ldots \ldots \ldots 54-118$

Appendix B. ARSA Questions Encountered During the EML Field Test . . $119-124$ 


\section{$\mathbf{I}_{\text {mmooctrox }}$}

A requirement of the Comprehensive Test Ban Treaty (CTBT) is to establish an international network of radionuclide monitoring stations to detect and locate nuclear explosions in all environments. To meet that requirement, the Proliferation and National Security R\&D Office of the U.S. Department of Energy (DOE) is to provide the research to develop the technologies for U.S. Government agencies responsible for compliance with the CTBT.

A part of the CTBT R\&D research effort is to develop high sensitivity instrumentation systems to detect and analyze radioxenon gas from nuclear testing. A major specification is that the system functions without operators so that it may be deployed to remote locations and be capable of making continuous measurements in a near-real time mode.

In response to the CTBT R\&D requirements, a prototype Automated Radioxenon Sampler/Analyzer (ARSA) System has been designed by the Pacific Northwest National Laboratory (PNNL). The Environmental Measurements Laboratory (EML) tested the instrument from late March to early December 1997 with the objective of determining if it conforms to CTBT specifications. The program encompassed a series of tests to determine the performance of the ARSA under simulated field conditions.

The program was an interagency, DOE interlaboratory and private sector cooperative effort sponsored by the DOE and AFTAC. The participants were the AFTAC/ Materials Technology Directorate (TM), AFTAC/Technical Operations Division (TOD), PNNL, EML and the British Oxygen Corporation (BOC).

AFTAC/TM was to provide oversight and coordinate the activities of the agencies involved in the field test, AFTAC/TOD was to determine the potential ARSA deficiencies, recommend improvements concerning maintenance/logistics issues and provide documentation of its evaluation in the final report.

The roles and responsibilities of PNNL were to: provide a prototype of the ARSA for testing; provide documentation, installation of the ARSA at the test site (EML); conduct initial inspection; fine-tune and transfer the instrument to EML control; supply technical staff or written detailed instructions should field repairs be necessary; furnish documentation and other 
information as requested by EML and arrange for pickup of the sampler at the conclusion of the program.

The major testing role was assigned to EML who was to: conduct a comprehensive field test of the field sampler as specified in the test plan; set up facilities for conducting the field test, including dedicated work space, phone lines, Internet, and electrical service; provide scientistlevel support for detailed performance testing; maintain daily logs of sampler performance, operations, modifications and repairs; provide technician level support for daily operations, limited repairs, and shipment of samples for analysis; write and distribute an interim and final test report.

The BOC was to analyze samples collected by the ARSA and report the results of the radioxenon concentrations and volumes of recovered xenon gas.

Installation (1997)

The ARSA and PNNL installation team arrived at EML on March 24. The system was located in the EML Environmental Test Chamber Facility that contains the necessary $208 \mathrm{~V}$ electrical service, local area network (LAN) connection, and dedicated telephone lines. Assembly was completed by March 28. A 3/4" copper line was installed between the ARSA and the Chamber to satisfy test requirements of providing sample air under controlled temperaturehumidity conditions and specified ${ }^{133} \mathrm{Xe},{ }^{222} \mathrm{Rn}$ concentration levels.

\section{PERATION (1997)}

ARSA startup, fine adjustments as well as training of EML personnel continued for an additional two-week period after assembly had been completed. The series of EML tests was started on April 14 and continued on schedule until May 2.

Testing was scheduled to span a 5-month period (March 24 to August 4). However, the tests were terminated on May 3 because of damage caused by an ARSA malfunction which led to an 
overheated system. After assessment of the damage, PNNL personnel decided that on-site repairs could not be made and the damaged assemblies (process deck) were returned to Richland, WA. PNNL estimated that approximately eight weeks would be required to restore the system to operating condition.

ARSA was reinstalled at EML in mid-July, after PNNL had made repairs and added or modified hardware and software safety features (see following section on ARSA Improvements Following the Smoke Condition Event). Testing was resumed in early August. All required tests were completed by December 3, when the system was disassembled and returned to PNNL.

\section{General Test Descriptions}

Forty-six individual tests designed to ascertain the operating characteristics and performance of the ARSA prototype under simulated field conditions were derived. Individual test names, test descriptions, objectives, requirements and execution are summarized in Table 1 . The Test Plan assigns each individual test into one of six main categories, Physical and General (PGT), Collection (CT), Analysis (AT), Data Storage and Communications (DCT), Operation/Logistics (OT) and Destructive Tests (DT).

The PGT's (12 tests) were intended to demonstrate that the system meets reasonable physical layout requirements, noise levels, temperature ranges, weight, power consumption, operation cost, etc.

The CTs (four tests) were designed to demonstrate that the unit is collecting xenon from the atmosphere over an 8-hour period, and to determine the extent of radon removal;

The AT's ( 9 tests) were intended to demonstrate the system's capability to reliably identify and measure all radioxenons of interests;

The DCT's (five tests) were to determine the ability for the system to reliably store and transmit data from the test site, and to allow remote control of the system;

The OT's (11 tests) were to demonstrate the system's simplicity of design, flexibility and robustness; 
The DT's (five tests) were not to be performed but required that PNNL provide an explanation of the anticipated results.

\title{
ENERAL THEORY OF SAMPLER OPERATION
}

\begin{abstract}
ARSA
Sample air, maintained at a flow rate of $100 \mathrm{~L} / \mathrm{min}$, is initially drawn through a 32 "long $x$ 6.0 " (id) trap containing a mixture of alumina and $13 \mathrm{X}$ molecular sieve to remove water and carbon dioxide. After chilling the sample air, radon is removed by passing the air stream through a 4 " long x $1.0^{\prime \prime}$ (id) pre-trap containing an activated charcoal trap at $-95^{\circ} \mathrm{C}$. Xenon is not adsorbed on the pretrap because of its small size and the short contact time of the flow stream with the adsorbent. However, xenon is quantitatively adsorbed in the following 11.0" long x 4.0" (id) activated charcoal trap (Main Trap) cooled to $-125^{\circ} \mathrm{C}$. After completion of 8-hours of sampling, the Main Trap is heated to $300^{\circ} \mathrm{C}$ and the xenon and other impurities are desorbed into a nitrogen carrier gas stream. The desorbed xenon is purified by passing the carrier gas through a series of three traps: the first trap ( $24^{\prime \prime} \times 4.0^{\prime \prime}$ id) containing a mixture of silica gel and Ascarite to remove traces of carbon dioxide; the second (6" x 1.0" id Post Trap) containing 5A molecular sieve to remove residual radon; and the last trap, the final charcoal trap ( $2.0^{\prime \prime} \times 0.25 "$ id), where the xenon is adsorbed at $<100^{\circ} \mathrm{C}$. Finally, xenon is thermally desorbed by heating the final charcoal trap to $\sim 450^{\circ} \mathrm{C}$, and transferring the desorbed gas into one of four evacuated scintillation detector cells, each with an internal volume of approximately $6.4 \mathrm{~mL}$. The gas in the counting cell is counted for 16-hours by beta-gamma coincidence counting, the resulting spectra are recorded, and the 31 and $81 \mathrm{keV}$ are peaks integrated. Appropriate detector efficiencies are applied to the count rates to convert to the absolute disintegration rates of the radioxenons in the sample. After completion of counting, the gas is analyzed by a residual gas analyzer (RGA) to determine the volume of recovered elemental xenon. Finally, the sample gas is expanded into an evacuated $150 \mathrm{~mL}$ archive bottle for confirmatory laboratory analysis.
\end{abstract}

All timed events (valve sequences, flow controllers, temperatures, detectors) are controlled by a Ziatech Modular Computer with a QNX operating system and PNNL coded software. All data are downloaded to a PC where RGA spectra, beta-gamma coincidence spectra and operating conditions are displayed via Graphical User Interface (GU) software. In case of power failure, an uninterruptible power supply (UPS) maintains data in the Ziatech computer memory. The 
system was accessed from PNNL and EML remote terminals through a LAN, however, no modem was provided for direct RS-232 telephone/modem communication.

A total of 29-hours is required to complete a sample cycle, each cycle is divided into a collection (8-hours), purification (5-hours) and radioassay (16-hours) phase.

To maintain a $100 \%$ duty cycle, the ARSA pneumatics consists of two identical sample collection and purification system trains programmed to alternate every 8 -hours. While one system is in the sample collection mode, the traps in the alternate system are in a bake-out regeneration mode to purge residual radioactive and non-radioactive contaminants.

As discussed earlier, ARSA contains a beta-gamma coincidence detector system to determine the concentration of the radioxenons in the sample. The system consists of four cells, each consisting of a 2 " length $\times 0.6^{\prime \prime}$ diameter cylinder of plastic scintillation material, all surrounded by two 7" 1 " 1 3" NaI(TI) crystals with associated photomultiplier tubes and multichannel analyzer electronics. Although the four cells are in a simultaneous counting mode for approximately 16 -hours, only two cells contain purified sample at any one time. The other two cells are on background count. For example, at time zero, Cell \#1 is filled with xenon gas from the purified air sample and the sample count is started for a period of 16-hours. Cell \#2, which has been on background count for 8-hours, enters the second 8-hours of the 16-hour counting period. Cell \#3, which has completed the 16-hour radioassay of a previously purified sample (the gas transferred to an archive bottle), and has been decontaminated under reduced pressure for approximately 10-minutes, begins its 16 -hour background radioassay. Cell \#4, which contains xenon gas from a previously processed sample, enters the second 8-hours of its 16 -hour counting period.

Complete details of the entire ARSA system have been previously published (Bowyer 1997). 


\section{A rsa improvements following the Smoke CONDITION EVENT}

Following the ARSA overheating smoke condition incident (Bowyer 1997) of May 3, the process deck was returned to PNNL for repair and modifications. Hardware corrective actions included the replacement of urethane foam insulation with non-combustible (Cerablanket) insulation rated to $1300^{\circ} \mathrm{C}$, new temperature rated valves that did not require heating, new internal thermocouples that resist shorting, a smoke detector, and a cut-off button for the uninterruptible power supply. Pressure relief valves were also added to vent any over pressure in the traps. Software safety modifications included the reconfiguring of the over temperature override and providing safety interlocks that are independent of the hardware. A robust safety checking software keeps the ARSA system in a safe state by monitoring pressures, temperatures and flow rates for abnormal conditions.

New buttons added to the GUI Toolbar, include: Analyze, Logviewer, Met Data, and Control. Modifications or additions were made to the SOH (State of Health) and Sensors software. The function of each button is described as follows.

Analyze: Calculates and displays the ${ }^{133} \mathrm{Xe}$ sample concentration. A browser is provided to select the desired sample and associated detector background beta-gamma spectrum's files. The RGA (xenon gas volume) analysis file for the sample is automatically selected. A warning is displayed if the sample contains radon contamination. This capability was inoperative during the test period.

Logviewer: Displays lines of system events and changes in system events. Lines highlighted in red notifies the operator of an unsafe or abnormal operating condition.

Met Data: Displays and records wind measurements, temperature, relative humidity and dew point. This capability was inoperative during the test period.

Control: Allows the operator to determine the real-time status of each trap.

Sensors: A display of the nitrogen bottle pressure was added. 
SOH (State of Health): A graphical plot of the nitrogen bottle pressure over date and time was added.

\section{A rchive bottle analysis}

The contents of each 8-hour collection were transferred to a corresponding BOC sample bottle for analysis. The sample and a log sheet containing sample information (sample date, sampling time and bottle number) were forwarded to BOC for elemental xenon and ${ }^{133} \mathrm{Xe}$ determinations. One ARSA bottle containing ${ }^{135} \mathrm{Xe}$ activity, as determined by beta-gamma coincidence counting, was express shipped to TOD/MCL, McClellan AFB for analysis.

\section{$\mathbf{R}_{\text {Esults }}$}

Test data and the results of each field test, in the specified AFTAC format, are shown in detail in Appendix A. Table 1 contains a summary of the results for each of the 46 tests. All ARSA sample information and analysis results are listed in Table 2. Detector cell backgrounds from randomly selected beta-gamma spectra are listed in Table 3.

Typical beta-gamma MCA spectra of ${ }^{133} \mathrm{Xe}$, with and without ${ }^{222} \mathrm{Rn}$ contamination $\left({ }^{214} \mathrm{~Pb}\right)$ peaks, are illustrated in Figures 1 and 2, respectively. Beta-gamma and gamma spectra of the ${ }^{154,155} \mathrm{Eu}$ standards are shown in Figures 3 and 4, respectively. Figure 5 is a plot of a typical RGA mass spectra. Figures 6 through 9 are graphical displays of ARSA integrated and instantaneous power measurements from a cold start and during continuous (8-hour) operation.

\section{ANALYSIS TESTS}

The requirement of Test AT1 was that the system should be capable of on-site radioxenon separation and measurement. RGA and laboratory analysis results verify that the system does extract xenon from atmospheric samples. Sample beta-gamma ray spectra also verify that the system does radioassay the radioxenons. However, although operating quantitation software was 
provided with the system, it could not be used because the correct algorithms had not been furnished to convert the counting data to sample radioxenon concentrations.

A detector background (AT2) is determined for each cell every 32-hours (see ARSA General Theory of Operation). Test results indicate a detector memory effect that is defined as the increase in the detector cell background from the residual activity remaining in the detector after the sample had been transferred to the archive bottle and the cell is evacuated under reduced pressure. The average percent residual ${ }^{133} \mathrm{Xe}$, beta-gamma background activity ( $\left.31 \mathrm{keV}\right)$ remaining in the detector cells after three samples (ranging from $2 \mathrm{cpm}$ to $21 \mathrm{cpm}$ ) had been radioassayed, was $3.6 \pm 0.5 \%$. The mean percent residual ${ }^{222} \mathrm{Rn}$ from six samples (three background and three samples from Test AT7), after samples with activity levels ranging from 6 to $829 \mathrm{cpm}$ had been radioassayed, was $11.1 \pm 3.5 \%$. All cell backgrounds obtained after August 15 were contaminated with ${ }^{222} \mathrm{Rn}$ (see Table 3). Table 3 backgrounds (total, $31 \mathrm{keV}, 81 \mathrm{keV}$, radon) were determined using the any_beta_gamma window. The ${ }^{222} \mathrm{Rn}$ count rates were obtained by the summation of the radon progeny $(242,295$, and $352 \mathrm{keV}){ }^{214} \mathrm{~Pb}$ peaks.

Sample count times are approximately 16-hours. To retrieve counting data (AT5) during a counting run, counting for that sample detector cell must be interrupted by issuing a stop and save command (followed by a file ID). The spectrum file is then saved on hard disk and can be viewed on the monitor. However, the saved data are no longer stored in the multichannel analyzer (MCA) memory. On resumption of counting, new data are accumulated and stored in the MCA until the next interruption or the end of the counting period. All of the saved data files must be summed (software not provided) to calculate the sample radioxenon concentration.

Counter calibrations (AT6) are determined by the radioassay of a $0.5 \mathrm{nCi}^{154,155} \mathrm{Eu}$ source provided for each detector cell. These sources are not absolute detector calibration sources but function as working standards to monitor the stability of the counting systems. Each source is pneumatically introduced and withdrawn from the detector cell through its own transfer line, located outside the detector cell. The counting reproducibility for each cell was determined by simultaneously introducing all four sources to its respective cell for 16-hours counting. The sources were withdrawn and reintroduced for another 16 hours of counting. The counting reproducibility for three complete cycles, was $<3.1 \%$. However, a decrease in the total counts was observed for detector cell number $3(\sim 75 \%)$ and number $4(\sim 25 \%)$ from those obtained for Test AT10 (Detector Cross-Talk). 
Radon is a major interference in the determination of the radioxenons in ambient air. Test AT7 was designed to determine the effectiveness of ${ }^{222} \mathrm{Rn}$ removal by the ARSA system. The

${ }^{222} \mathrm{Rn}$ concentration in the EML Environmental Chamber was set to provide a sample intake stream containing twice the highest recorded ambient level $(3.0 \mathrm{pCi} / \mathrm{L})$. Test results $(3.8 \mathrm{pCi} / \mathrm{L}$, measured) indicate that radon is separated from xenon, but retained by the purification traps and eluted into the next sample processed through those traps.

The required minimum detectable levels (MDL) for ${ }^{131 \mathrm{~m}} \mathrm{Xe},{ }^{133 \mathrm{~m}} \mathrm{Xe},{ }^{133 g} \mathrm{Xe}$ and ${ }^{135} \mathrm{Xe}$ for a 8hour cycle time are specified in Test AT8. PNNL calculated results (Reeder et al. 1997) incorporate the effects from ${ }^{222} \mathrm{Rn}$ interferences. EML's calculations are based on clean baseline background spectra (any_beta_gamma window) for 8-hour collection cycle times. Both results are based on 16-hours of radioassay. Regardless, it appears that under the worst case conditions $\left({ }^{222} \mathrm{Rn}\right.$ contamination), the ARSA system meets the test specifications.

Test AT9 (Detector Cross-Talk) was not in the original test plan but was added at a later date. Since the four detector cells are in a simultaneous radioassay mode, this test was to ascertain if a radioactive source in one detector cell contributes to the count rate of the other three cells. A system ${ }^{154,155} \mathrm{Eu}$ calibration source was separately counted in each cell while the backgrounds of the other cells were simultaneously determined. Detector crosstalk ranged from $0.5 \%$ to $22.8 \%$ of the activity in the reference cell.

\section{COLLECTION TESTS}

For the series of four Collection Tests, the Collection Time (CT2) and Xenon Gas Yield (CT3) tests met the specifications. However, the CT3 requirement was met only after the RGA had been relocated and the product transfer line modified. The mean ratio of xenon RGA/laboratory analysis for 94 samples collected after RGA relocation was $0.61 \pm 0.09(14.7 \%)$, indicating consistent RGA agreement with the analysis results. A ratio of 1.0 would not be expected because of losses (v/v) from sample transfer to the analysis bottle.

The Duty Cycle (CT1) test requires that ARSA operate at a $100 \%$ duty cycle. We understand that the main charcoal trap does not attain the desired subambient temperature for approximately one-half hour after the start of each eight-hour collection cycle. This implies that xenon is not quantitatively adsorbed on the trap during that time period and that ARSA operates at a 94\% duty cycle. 
Two separate experiments were run to determine the system's efficiency of purging residual gas (CT4). The first experiment was run by bringing the air volume of the EML Environmental Chamber to a ${ }^{133} \mathrm{Xe}$ concentration of $20 \mathrm{pCi} / \mathrm{SCM}$, followed by the sampling of chamber air for 8 hours. A steady state ${ }^{133} \mathrm{Xe}$ concentration was maintained in the chamber by the addition of sufficient ${ }^{133} \mathrm{Xe}$ activity (ca) to make up for the activity removed by the sampling systems. A chamber port was opened to replace the volume of air removed by the sampling systems. Ambient air was collected after the last radioxenon sampling cycle to determine the system's ${ }^{133} \mathrm{Xe}$ purging efficiency. The mean (six samples) of the ARSA analytical results at the $20 \mathrm{pCi}$ level was $7.5 \pm 1.6 \mathrm{pCi} / \mathrm{SCM}$, the difference from the expected caused by the introduction of room air into the sample stream through an unexpected opening in the ARSA sample intake manifold. The manifold was repaired for the second $(100 \mathrm{pCi} / \mathrm{SCM})$ experiment when three ARSA samples (mean $88.5 \pm 8.3 \mathrm{pCi} / \mathrm{SCM}$ ) were collected. The percentages of residual ${ }^{133} \mathrm{Xe}$ activities were 3.2 $\pm 1.1 \%$ (@7.5 pCi ) and 4.5 0.2\% (@88.5 pCi). These values are in good agreement with those obtained for the Test AT2 detector background memory effect (3.6\%), which suggest that the residual ${ }^{133} \mathrm{Xe}$ activity may not be due to incomplete purging of the ARSA purification system.

\section{DATA STORAGE AND COMMUNICATIONS TESTS}

Of the five tests, the Communications Test (DCT3) was deleted from the test plan because a direct RS-232 telephone/modem communication had not been provided. All remote accessibility observations for the remaining tests were made through LAN communications only. However, LAN proved to be extremely slow, particularly when accessing stored files for graphical display.

Spectral and RGA files are identified by the end date and time of radioassay with either an .rga, sam or .bkg extension followed by the detector cell number. Test DCT2 requires that the system record the collection and analysis start/stop times for each sample. PNNL modified their software to satisfy the test requirements by creating a sample summary file which correlates the file identification number with the start of the collection cycle.

\section{DESTRUCTIVE TESTS}

The destructive tests were not run, as specified in the test plan. Detailed opinions (PNNL) as to the worst effects on ARSA operations from a blocked airstream (DT1), water ingestion (DT2), drain clogs (DT3), flooding (DT4), and voltage spikes (DT5) are reported on the individual test records (see Appendix A) and are summarized in Table 1. 


\section{OPERATION AND LOGISTICS TESTS}

The user's manual described under OT2 has not been updated. In particular, system and software modifications made after the overheating incident should be added.

The prototype was incapable of unattended operation (OT3) over a long period of time. The system was subjected to numerous shutdowns for repairs or delays to adjust operating parameters throughout the test period.

\section{PHYSICAL AND GENERAL TESTS}

Four of the 12 tests did not meet the specifications. ARSA exceeded the size specification (PGT1), total system weight (PGT2), noise level (PGT3) and power draw (PGT12).

Two tests were proposed to determine the capability of the system to operate in a temperature environment ranging from $50^{\circ}$ to $100^{\circ} \mathrm{F}$ (PGT4) and a humidity environment from $0 \%$ to $100 \%$ at $75^{\circ} \mathrm{F}$ (PGT7). It was originally intended to operate the system in the Environmental Chamber where the temperature and humidity conditions could be controlled. However, the entire unit did not fit through the chamber access doors and would have had to be disassembled and reassembled in the chamber. The test was modified to determine the operational effects at recorded room temperature and humidity conditions. No malfunctions, damage to the components or changes in analytical functions were attributed to room temperature $\left(65^{\circ}\right.$ to $\left.84^{\circ} \mathrm{F}\right)$ or room humidity (15\% to $83 \%)$ conditions.

Xenon yields appear to be affected by sample air stream humidity. Although the air humidity in the Environmental Chamber was set as prescribed by Test PGT6, the intake air stream humidity was compromised by the air leak in the ARSA sample manifold. The mean xenon recovery (RGA) for five samples collected with the air humidity in Environmental Chamber at $20 \%$ and $50 \%$, was $1.76 \mathrm{~mL}$. Xenon RGA recoveries for the initial two samples collected at $90 \%$ humidity was $1.90 \mathrm{~mL}$, but the volume of collected xenon decreased to a mean of $1.30 \mathrm{~mL}$ for the following nine samples. 


\section{ONCLUSIONS AND RECOMMENDATIONS}

The ARSA prototype is a state-of-the-art system designed to separate and radioassay the radioxenons in the atmosphere. The PNNL software to monitor ARSA operations is impressive, user friendly and excellent. Toolbar buttons give accessibility to the real time status of the hardware components and detector system. Different windows may be accessed to display the temperature of each adsorbent trap, as well as the status of the dryers, valves, devices (compressor, RGA, etc.), UPS, analog board, and AC power. Other windows display the pressures and gas flows. The Logviewer window, which displays lines of system events is a valuable safety and diagnostic feature. We recommend that the ARSA software be considered for the next generation systems.

We were also impressed with the detector's design and the rationale of beta-gamma coincidence radioassay. The system's low backgrounds and high detector efficiencies makes the measurement of extremely low levels of the radioxenons in the atmosphere possible, a prime consideration for its use in the CTBT global network. However, we were disappointed with the detector's memory (radioxenons and radon) and crosstalk effects. The memory effect is particularly troublesome in that it could impede the determination of a sample with background radioxenon concentrations if it follows one with high concentrations.

With regard to radon contamination, all samples processed after August 15 were contaminated with radon activities, but not all samples processed prior to May 2 were contaminated. This suggests that ARSA is capable of providing radon free samples.

As with any prototype system, one would expect shutdowns for repairs or modifications. As stated earlier, ARSA was subject to numerous shutdowns due to hardware component failures. We have not recorded the changes in operating conditions made by PNNL via their remote terminal. Based on discussions with PNNL technical staff, it is our impression that the system was continually fine tuned to keep the system operational and on the test schedule.

PNNL rapidly provided technical personnel and replacement parts after shutdowns from hardware failures. Their personnel were competent, cooperative and professional.

Based on the deficiencies in the test results, we propose the following recommendations for improved performance. 


\section{DETECTOR MEMORY EFFECT (AS DEFINED)}

Ideally, to maximize the lower limits of detection and minimize the propagation of the uncertainties of the measurement, the detector cells should be completely decontaminated before sample introduction.

\section{POWER CONSUMPTION}

ARSA power consumption far exceeds the specification. It would be advantageous to investigate the use of components that require less power. For example, smaller traps could be rapidly brought to the desorption temperature by ohmic heating.

\section{NITROGEN CARRIER GAS}

Based on the amount of nitrogen carrier gas consumed during the test period, six (6), $1 \mathrm{~A}$ cylinders/year would be required This may be a problem for a system located at a remote site. It may be advisable to install a nitrogen gas generator, if power consumption could be first reduced.

\section{CONDENSATE TRAP}

We were required to manually drain a condensate trap located in the process deck at intervals of approximately every seven days of continuous running. An automatic drain should be installed.

\section{XENON RECOVERY AT 90\% SAMPLE AIR HUMIDITY}

Determine and remedy the cause of the decrease in xenon recovery $(\sim 25 \%)$ from sample air at high humidity.

\section{HARDWARE FAILURES}

We experienced a considerable number of shutdowns from hardware component malfunctions, particularly thermocouples and solenoid valves, during the entire test period. They should be replaced with more reliable parts. 
An alternate means of communication should be found to improve the response time for acquiring information or changing operating instructions from a remote terminal.

\section{RADON RETENTION}

A procedure or system modification should be made assuring that radon is quantitatively purged from the adsorbent traps.

\section{GRAPHICAL OUTPUT}

Provide a means (screen dump) of printing the graphical displays of RGA and MCA spectra.

\section{DETECTOR CROSS TALK}

The detector system should be further tested for crosstalk effects by using radioxenon gas as an internal detector source. The ${ }^{154,155} \mathrm{Eu}$ sources should be relocated to minimize detector crosstalk.

\section{VARIABLE COUNT TIME}

Operate the system as a typical commercially available MCA, so that beta-gamma spectra may be viewed on the monitor without interfering with counting.

\section{ARSA SIZE}

The unit exceeds the footprint specifications. Reduce the size to meet specifications.

\section{ARSA WEIGHT}

Total system weight exceeds 1000 pounds. Detector shield (lead and copper) weighs approximately 1335 pounds. Determine if the shielding could be reduced without increasing the detector backgrounds. 


\section{RADIOXENON QUANTITATION SOFTWARE}

Provide the correct branching ratios, detector efficiencies and standard operating procedures to determine the near real-time air concentrations of all the radioxenons. Compare the results with laboratory analysis results.

\section{COMMUNICATIONS}

Provide modem communications via the RS-232 port.

\section{NOISE LEVEL}

The system's noise level exceeds the specifications. Add sound deadening material to reduce the noise level. 


\section{References}

Bowyer, T. W.

Automated Xenon Sampler-Analyzer User's Manual, Pacific Northwest Laboratory, January 7 (1997)

Bowyer, T. W., K. H. Abel, M. E. Panisko and R. C. Thompson

ARSA Malfunction at EML, Pacific Northwest National Laboratory, (Private Communication) May 7 (1997)

Reeder P. L., T. W. Bowyer and K. H. Abel Analysis of Beta-Gated Gamma Spectra for the PNNL ARSA and Estimate of Minimum Detectable Activities for $\left({ }^{131 \mathrm{~m}} \mathrm{Xe}+{ }^{133 \mathrm{~m}} \mathrm{Xe}\right),{ }^{133 \mathrm{~g}} \mathrm{Xe}$, and ${ }^{135 \mathrm{~g}} \mathrm{Xe}$ Pacific Northwest National Laboratory (Private Communication), December 2 (1997) 
TABLE 1

SUMMARY OF TEST DESCRIPTIONS, OBJECTIVES AND RESULTS

\begin{tabular}{|c|l|l|l|l|}
\hline TEST & \multicolumn{1}{|c|}{ NAME } & \multicolumn{1}{|c|}{ OBJECTIVE } & \multicolumn{1}{|c|}{ EXECUTION } & \multicolumn{1}{c|}{ RESULTS } \\
\hline AT-1 & $\begin{array}{l}\text { On-Site } \\
\text { Measurement }\end{array}$ & $\begin{array}{l}\text { The system should be } \\
\text { capable of on-site } \\
\text { radioxenon separation } \\
\text { and measurement. }\end{array}$ & $\begin{array}{l}\text { Operate the system under normal } \\
\text { conditions. }\end{array}$ & $\begin{array}{l}\text { - Radioxenon separated, radioassayed, but } \\
\text { not calculated. }\end{array}$ \\
$\begin{array}{l}\text { Initially, low elemental xenon recoveries. } \\
\text { Recoveries between 1.0-2.0 mL after } \\
\text { relocation of RGA. }\end{array}$ \\
$\begin{array}{l}\text { Anitially, RGA elemental xenon recoveries } \\
\text { disagreed with analysis results. Agreement } \\
\text { after relocation of RGA. }\end{array}$
\end{tabular}


TABLE 1 (Cont'd)

\begin{tabular}{|c|c|c|c|c|}
\hline TEST & NAME & OBJECTIVE & EXECUTION & RESULTS \\
\hline AT -4 & $\begin{array}{l}\text { Gamma Ray } \\
\text { Spectrum Range }\end{array}$ & $\begin{array}{l}\text { Spectrum range should } \\
\text { be between } 20 \mathrm{keV} \text { to } \\
512 \mathrm{keV} \text {. }\end{array}$ & $\begin{array}{l}\text { Determine that the system is } \\
\text { performing the required } \\
\text { measurements within the specified } \\
\text { energy range by inspecting } \\
\text { transmitted and archived data files. }\end{array}$ & $\begin{array}{l}\text { - Experimental spectrum range is } 15 \mathrm{keV} \text { to } \\
440 \mathrm{keV} \text {. Satisfactory data provided from } \\
\text { archived files. } \\
\text { - Transmitted data file test deleted from the } \\
\text { plan. }\end{array}$ \\
\hline AT -5 & $\begin{array}{l}\text { Variable Count } \\
\text { Time }\end{array}$ & $\begin{array}{l}\text { Remotely retrieve } \\
\text { spectra without } \\
\text { interfering with the } \\
\text { counting times. }\end{array}$ & $\begin{array}{l}\text { Determine at } 1,4,10 \text { and } 15 \mathrm{~h} \text { and } \\
\text { verify proper operation by } \\
\text { inspecting the data files. }\end{array}$ & $\begin{array}{l}\text { - Counting must be stopped for the detector } \\
\text { cell of interest and the counting data in the } \\
\text { detector computer memory saved on the PC } \\
\text { disk. Computer must be instructed to } \\
\text { resume counting, but previous counting } \\
\text { data not stored in MCA memory. Counting } \\
\text { restarted at time zero and continues until } \\
\text { the end of the programmed run. Spectral } \\
\text { files must be summed for quantification. }\end{array}$ \\
\hline AT-6 & $\begin{array}{l}\text { Efficiency } \\
\text { Calibration }\end{array}$ & $\begin{array}{l}\text { The instrument should } \\
\text { have a means to } \\
\text { remotely measure the } \\
\text { relative detector } \\
\text { efficiency. }\end{array}$ & $\begin{array}{l}\text { Determine that the system is } \\
\text { performing the efficiency } \\
\text { measurements and calculations by } \\
\text { inspecting the files. }\end{array}$ & $\begin{array}{l}\text { - Detector efficiencies previously determined } \\
\text { at PNNL. } \\
\text { - Secondary }\left({ }^{154,155} \mathrm{Eu}\right) \text { standards provided for } \\
\text { each detector cell to determine counter } \\
\text { stability }(<3.1 \%) \text {. }\end{array}$ \\
\hline AT-7 & Radon & $\begin{array}{l}\text { The system shall have } \\
\text { a way to reduce radon } \\
\text { contamination so it } \\
\text { does not interfere with } \\
\text { the measurement of the } \\
\text { isotopes of interest. }\end{array}$ & $\begin{array}{l}\text { Determine that the system is } \\
\text { performing the required reduction of } \\
\text { radon interference at twice the } \\
\text { highest recorded atmospheric value } \\
(3 \mathrm{pCi} / \mathrm{L}) \text {. Evaluated spectral data } \\
\text { to determine if radon breakthrough } \\
\text { occurs. }\end{array}$ & $\begin{array}{l}\text { - Air sampled from the environmental } \\
\text { chamber containing } 3.8 \pm-0.2 \mathrm{pCi}^{222} \mathrm{Rn} \text {. } \\
\text { Three } 8.0 \text {-h samples collected. } \\
\text { - System contains two separate gas streams. } \\
\text { Increase in }{ }^{222} \mathrm{Rn} \text { activity ranged from a } \\
\text { factor of } 15 \text { to } 39 \text { for samples processed } \\
\text { successfully through gas stream " } 0 \text { " and } \\
\text { "1", respectively. }\end{array}$ \\
\hline
\end{tabular}


TABLE 1 (Cont'd)

\begin{tabular}{|c|c|c|c|c|}
\hline TEST & NAME & OBJECTIVE & EXECUTION & RESULTS \\
\hline AT-8 & $\begin{array}{l}\text { Xenon } \\
\text { Minimum } \\
\text { Detectable } \\
\text { Levels }\end{array}$ & $\begin{array}{l}\text { Determine the minium } \\
\text { detectable levels for a } \\
\text { 6-h cycle time. }\end{array}$ & $\begin{array}{l}\text { Calculate the minimum detectable } \\
\text { levels using the counting efficiency, } \\
\text { background counts, and the volume } \\
\text { of air sampled. }\end{array}$ & 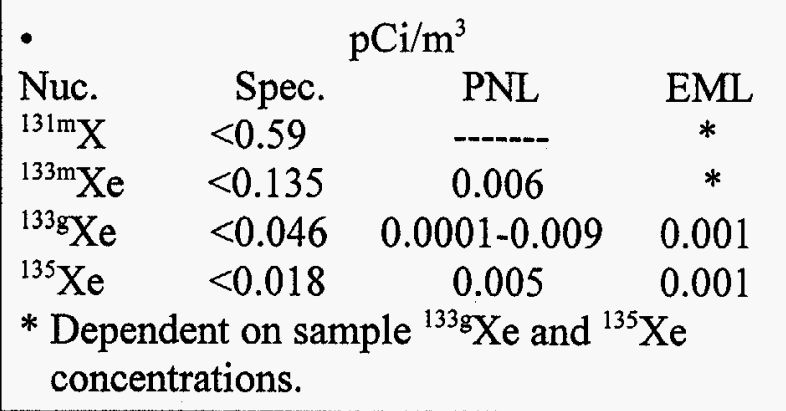 \\
\hline AT-9 & $\begin{array}{l}\text { Detector } \\
\text { Crosstalk }\end{array}$ & $\begin{array}{l}\text { Determine the } \\
\text { contribution to detector } \\
\text { background (crosstalk) } \\
\text { from the internal } \\
\text { calibration sources. }\end{array}$ & $\begin{array}{l}\text { Count the }{ }^{154,155} \mathrm{Eu} \text { calibration } \\
\text { source in each detector. Determine } \\
\text { the increase in background for the } \\
\text { adjacent detectors. }\end{array}$ & $\begin{array}{l}\text { - Contribution to the adjacent detectors } \\
\text { ranged from } 1.0 \text { to } 23 \% \text { of the total counts } \\
\text { from the reference detector. }\end{array}$ \\
\hline CT-1 & Duty Cycle & $\begin{array}{l}\text { The system should } \\
\text { demonstrate } 100 \% \text { duty } \\
\text { cycle, i.e., air } \\
\text { collection should be } \\
\text { continuous. }\end{array}$ & $\begin{array}{l}\text { Operate the system under normal } \\
\text { conditions and determine the duty } \\
\text { cycle. }\end{array}$ & $\begin{array}{l}\text { - Xenon is not quantitatively adsorbed on the } \\
\text { charcoal trap for } 30 \text { minutes of each } 480 \\
\text { min collection cycle. Duty cycle @ } 94 \% \text {. }\end{array}$ \\
\hline $\mathrm{CT}-2$ & Collection Time & $\begin{array}{l}\text { The time required to } \\
\text { collect the minimum } \\
\text { amount of xenon } \\
\text { should be no less than } \\
6 \mathrm{~h} \text { and no more than } \\
24 \mathrm{~h} .\end{array}$ & $\begin{array}{l}\text { Report the time required to } \\
\text { complete one collection cycle. }\end{array}$ & $\begin{array}{l}\text { - Eight hours required to collect sample air } \\
\text { (@ } 100 \mathrm{~L} / \mathrm{min} \text { ). } \\
\text { - Five hours to purify sample. } \\
\text { - Sixteen hours of radioassay time. } \\
\text { - Total elapsed time from start of sampling } \\
\text { to quantitation is } 29 \text {-hours. }\end{array}$ \\
\hline
\end{tabular}


TABLE 1 (Cont'd)

\begin{tabular}{|c|c|c|c|c|}
\hline TEST & NAME & OBJECTIVE & EXECUTION & RESULTS \\
\hline CT-3 & $\begin{array}{l}\text { Xenon Gas } \\
\text { Yield }\end{array}$ & $\begin{array}{l}\text { The system should } \\
\text { measure the total } \\
\text { amount of elemental } \\
\text { xenon collected in each } \\
\text { cycle. }\end{array}$ & $\begin{array}{l}\text { Analyze archive bottle contents } \\
\text { from } 5 \text { collection cycles. Verify that } \\
\text { equipment and algorithms used to } \\
\text { determine the total amount of } \\
\text { elemental xenon are working } \\
\text { correctly. }\end{array}$ & $\begin{array}{l}\text { - Initial wide differences between laboratory } \\
\text { analysis and ARSA RGA results of } \\
\text { elemental xenon collected. } \\
\text { - Constant ratio of } 0.61 \pm 0.09(14.7 \%) \\
\text { between laboratory analysis and RGA ( } 94 \\
\text { samples) obtained after relocation of the } \\
\text { RGA. }\end{array}$ \\
\hline CT-4 & System Purge & $\begin{array}{l}\text { The system should } \\
\text { completely purge all } \\
\text { residual gas after each } \\
\text { collection cycle. }\end{array}$ & $\begin{array}{l}\text { Operate the ARSA through } 24 \text { hours } \\
\text { while introducint }{ }^{133} \mathrm{Xe} \text { sufficient to } \\
\text { generate a } 20 \mathrm{pCi} / \mathrm{SCM} \text { average } \\
\text { intake level. For the next } 24 \text { hours, } \\
\text { feed the ARSA outside air while } \\
\text { simultaneously measuring the } \\
\text { outside air }{ }^{1338} \text { Xe level by laboratory } \\
\text { analysis. Measure any cross-cycle } \\
\text { bleed-over by analyzing system data } \\
\text { files and comparing to laboratory } \\
\text { analysis data. Repeat test at a } 100 \\
\text { pCi/SCM average intake. }\end{array}$ & $\begin{array}{l}\text { - @ } 5.2 \text { to } 9.2 \mathrm{pCi} / \mathrm{SCM} \text { - retention ( } 6 \\
\text { samples) averaged } 3.2 \pm 1.1 \% \text { of the added } \\
\text { activity. } \\
\text { - @ } 100 \mathrm{pCi} / \mathrm{SCM} \text { - retention ( } 3 \text { samples, } \\
\text { analysis mean }=88.5 \pm 8.3 \mathrm{pCi} / \mathrm{SCM} \text { ) was } \\
4.5 \pm 0.2 \% \text { of the added activity. }\end{array}$ \\
\hline DCT-1 & $\begin{array}{l}\text { Spectral } \\
\text { Information }\end{array}$ & $\begin{array}{l}\text { The system should be } \\
\text { capable of recording } \\
\text { sample spectral } \\
\text { information for each } \\
\text { cycle. }\end{array}$ & $\begin{array}{l}\text { Conduct normal operation of the } \\
\text { sampler. Determine that the system } \\
\text { is performing the required storage of } \\
\text { data by remotely requesting this } \\
\text { information for previously analyzed } \\
\text { data. Ensure that stored data is } \\
\text { correct. }\end{array}$ & $\begin{array}{l}\text { - Files acquired via EML's remote monitor } \\
\text { (LAN) } \\
\text { - Modem acquisition deleted from test plan. }\end{array}$ \\
\hline
\end{tabular}


TABLE 1 (Cont'd)

\begin{tabular}{|c|c|c|c|c|}
\hline TEST & NAME & OBJECTIVE & EXECUTION & RESULTS \\
\hline DCT-2 & $\begin{array}{l}\text { Collection } \\
\text { Information }\end{array}$ & $\begin{array}{l}\text { The system shall record } \\
\text { the collection and } \\
\text { analysis start and stop } \\
\text { times for each sample. }\end{array}$ & $\begin{array}{l}\text { Conduct normal operation of } \\
\text { sampler. Remotely request start and } \\
\text { stop times for previously analyzed } \\
\text { data. }\end{array}$ & $\begin{array}{l}\text { - Start times are not recorded and must be } \\
\text { calculated. } \\
\text { - End of collection time (PST) used in } \\
\text { filenames. Changed to local time after } \\
8 / 15 \text {. } \\
\text { - Created ample summary file which lists all } \\
\text { sample start times with sample end times. }\end{array}$ \\
\hline DCT-3 & Communications & $\begin{array}{l}\text { The data stored in the } \\
\text { system should be } \\
\text { provided to a serial } \\
\text { port for } \\
\text { communication. }\end{array}$ & $\begin{array}{l}\text { Conduct normal operation of } \\
\text { sampler. Determine that the system } \\
\text { is providing data via a standard RS- } \\
232 \text { serial port by monitoring its } \\
\text { signals. }\end{array}$ & $\begin{array}{l}\text { - Communication through a serial port } \\
\text { (modem, RS-232) not provided. } \\
\text { - Test deleted from plan. }\end{array}$ \\
\hline DCT-4 & $\begin{array}{l}\text { Remote } \\
\text { Programmability }\end{array}$ & $\begin{array}{l}\text { Determine that the } \\
\text { system can be remotely } \\
\text { programmed by } \\
\text { modifying operating } \\
\text { instructions. }\end{array}$ & $\begin{array}{l}\text { Inspect the transmitted and archived } \\
\text { data files. }\end{array}$ & $\begin{array}{l}\text { All remote programming via LAN. } \\
\text { - Remote programming via modem deleted } \\
\text { from test plan. }\end{array}$ \\
\hline DCT-5 & Data Storage & $\begin{array}{l}\text { Unit shall record } \\
\text { background, } \\
\text { calibration, and sample } \\
\text { spectral information } \\
\text { separately for each } \\
\text { collection/analysis } \\
\text { cycle. }\end{array}$ & $\begin{array}{l}\text { Determine that the system is } \\
\text { performing the required data storage } \\
\text { by inspecting the transmitted and } \\
\text { archived data files, calibrations, and } \\
\text { sample spectral information for each } \\
\text { cycle. }\end{array}$ & $\begin{array}{l}\text { - System recorded all sample spectral } \\
\text { information, background, and calibrations. } \\
\text { - Transmitted files test deleted from the test } \\
\text { plan. }\end{array}$ \\
\hline
\end{tabular}


TABLE 1 (Cont'd)

\begin{tabular}{|c|c|c|c|c|}
\hline TEST & NAME & OBJECTIVE & EXECUTION & RESULTS \\
\hline DT-1 & $\begin{array}{l}\text { Airstream } \\
\text { Blocked }\end{array}$ & $\begin{array}{l}\text { Input or output } \\
\text { airstream blocked. } \\
\text { Test not actually } \\
\text { conducted. }\end{array}$ & $\begin{array}{l}\text { PNNL: Provided explanation of } \\
\text { anticipated results of this test. } \\
\text { TOD: Examine PNNL rationale and } \\
\text { provide independent judgement or } \\
\text { test consequences. Report } \\
\text { independent evaluation. }\end{array}$ & $\begin{array}{l}\text { - PNNL: Compressor temperature would } \\
\text { increase and system would shut down } \\
\text { before overheating. It is anticipated that } \\
\text { system would not be damaged. } \\
\text { - TOD: State-of-health software would } \\
\text { recognize decrease in flow rate of } \\
\text { temperature. System would not be } \\
\text { damaged. }\end{array}$ \\
\hline DT-2 & Water Ingestion & $\begin{array}{l}\text { Input ingests } \\
\text { significant quantity of } \\
\text { water ( } 1 \text { gallon or } \\
\text { more). Test is not } \\
\text { actually conducted. }\end{array}$ & $\begin{array}{l}\text { PNNL: Provided explanation of } \\
\text { anticipated results of this test. } \\
\text { TOD: Examine PNNL rationale and } \\
\text { provide independent judgement or } \\
\text { test consequences. Report } \\
\text { independent evaluation. }\end{array}$ & $\begin{array}{l}\text { - PNNL: Compressor pressure would be } \\
\text { affected and system would shut down as in } \\
\text { DT-1. } \\
\text { - TOD: Compressor would most likely be } \\
\text { damaged. No damage to other components } \\
\text { in the sample flow path. }\end{array}$ \\
\hline DT-3 & Drain Clogs & $\begin{array}{l}\text { Water drain (from } \\
\text { dryer column) clogs. } \\
\text { Test is not actually } \\
\text { conducted. }\end{array}$ & $\begin{array}{l}\text { PNNL: Provided explanation of } \\
\text { anticipated results of this test. } \\
\text { TOD: Examine PNNL rationale and } \\
\text { provide independent judgement or } \\
\text { test consequences. Report } \\
\text { independent evaluation. }\end{array}$ & $\begin{array}{l}\text { - PNNL: System would not be damaged. } \\
\text { - TOD: System would shutdown before any } \\
\text { serious damage to the system. }\end{array}$ \\
\hline DT-4 & Flooding & $\begin{array}{l}\text { Base of unit is flooded } \\
\text { to } 6 \text { inches and } 12 \\
\text { inches. Test is not } \\
\text { actually conducted. }\end{array}$ & $\begin{array}{l}\text { PNNL: Provided explanation of } \\
\text { anticipated results of this test. } \\
\text { TOD: Examine PNNL rationale and } \\
\text { provide independent judgement or } \\
\text { test consequences. Report } \\
\text { independent evaluation. }\end{array}$ & $\begin{array}{l}\text { PNNL: Room flood from } 6 \text { " to } 12 \text { " water, } \\
\text { system would blow fuses. In deep floods, } \\
\text { low positioned equipment such as the } \\
\text { detection system, vacuum pump and } \\
\text { compressor would be severely damaged. } \\
\text { - TOD: Circuit breakers would be tripped } \\
\text { and or fuses blown. Immersed equipment } \\
\text { could be damaged. }\end{array}$ \\
\hline
\end{tabular}




\begin{tabular}{|c|c|c|c|c|}
\hline & 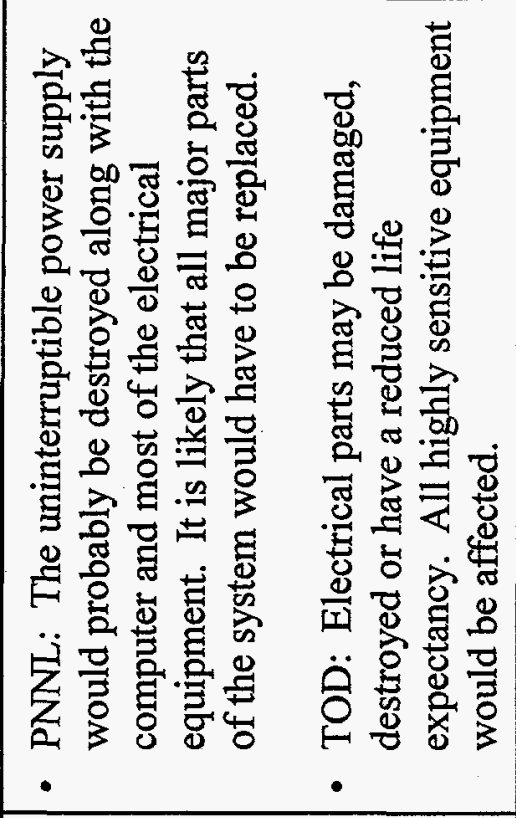 & 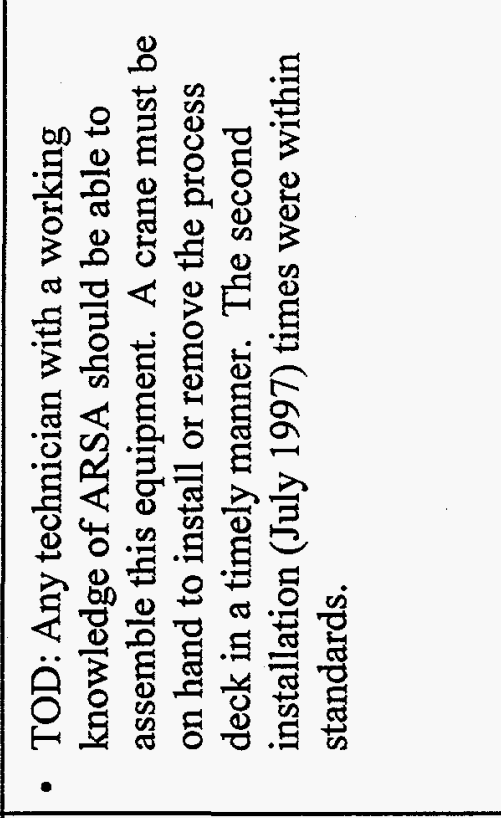 & 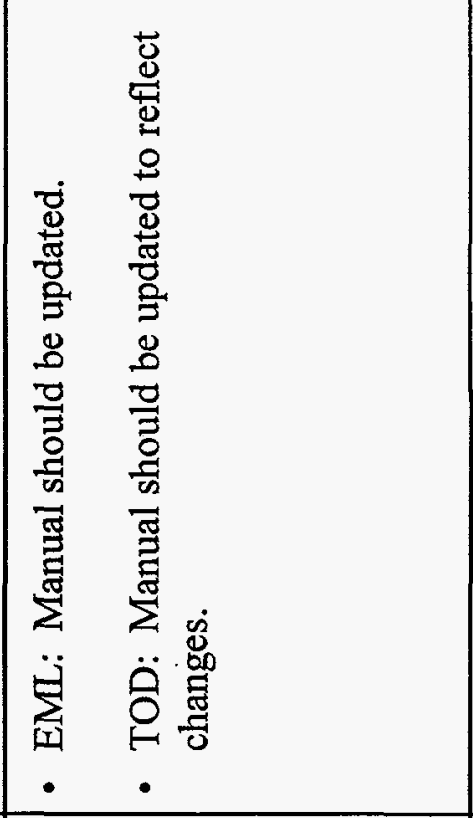 & 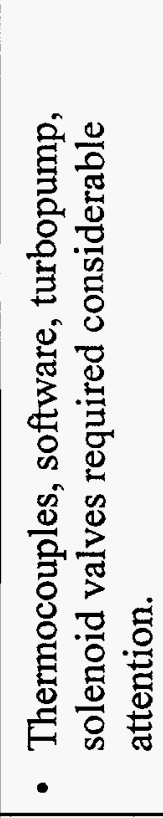 \\
\hline 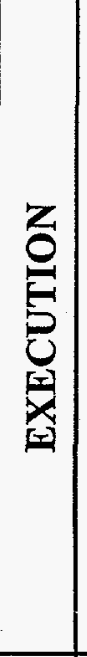 & 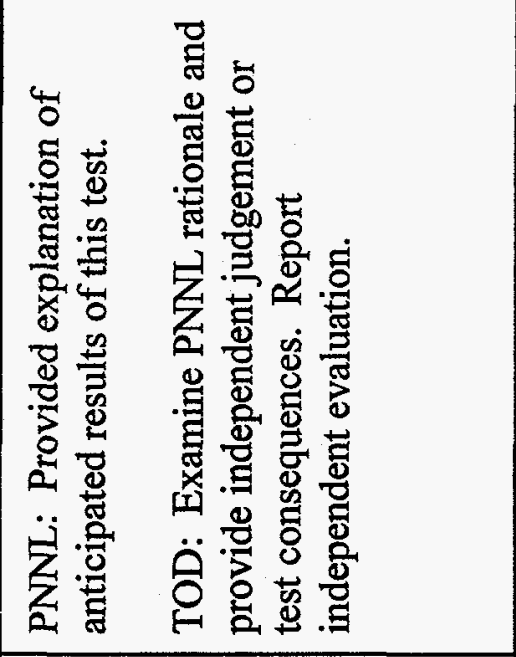 & 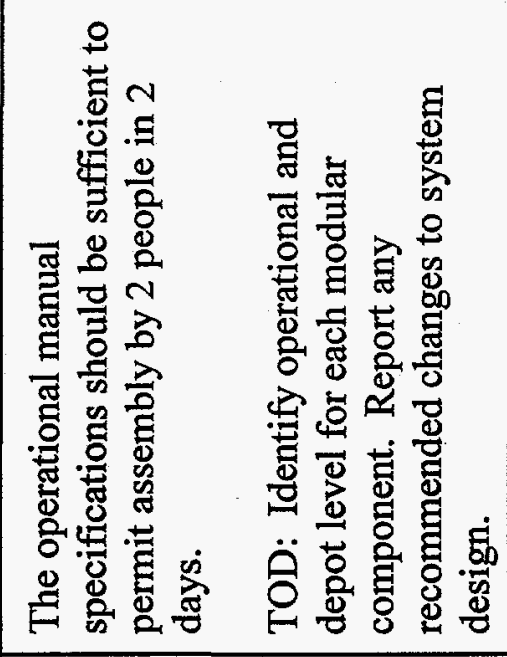 & 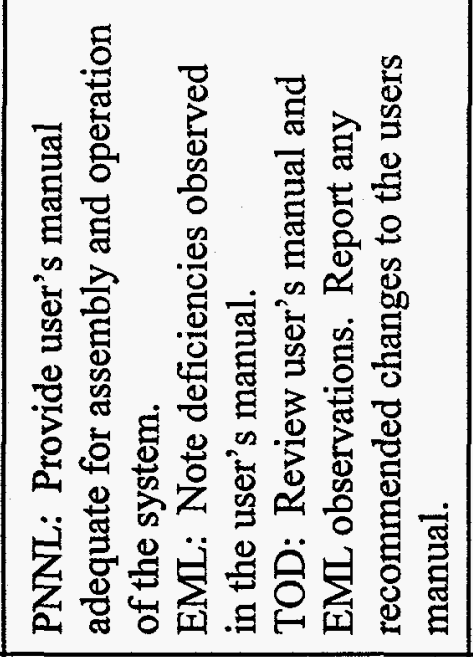 & 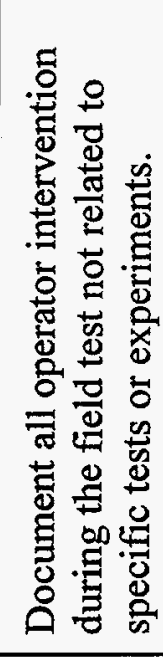 \\
\hline 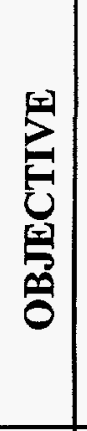 & 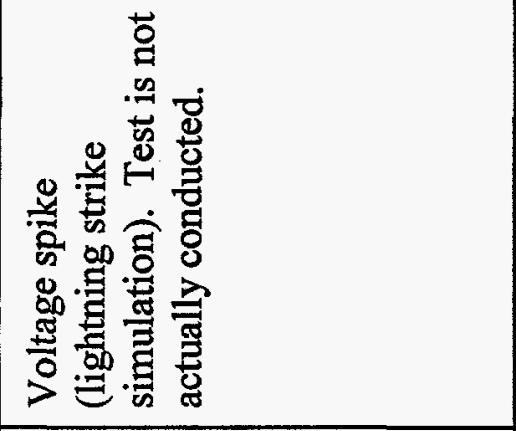 & 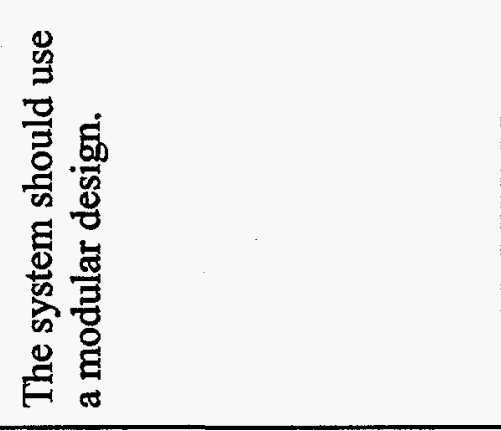 & 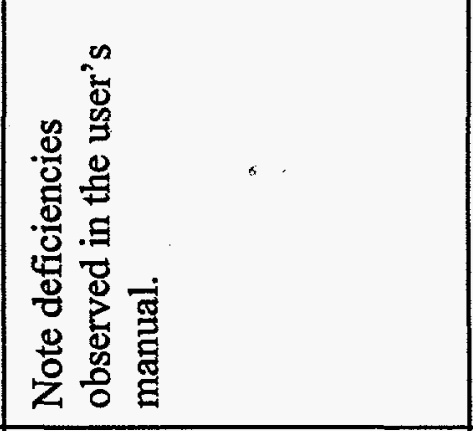 & 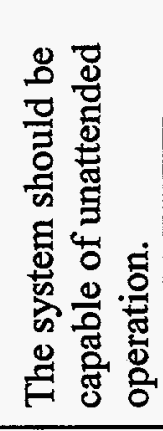 \\
\hline 鼠 & 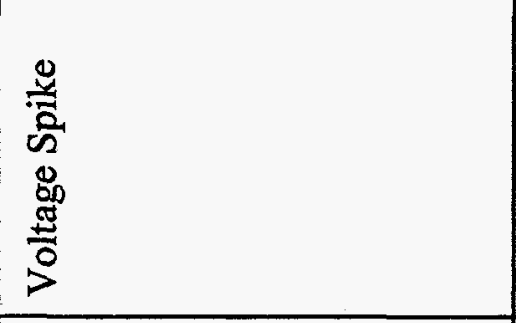 & 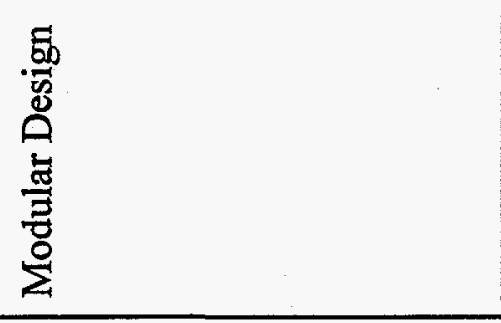 & 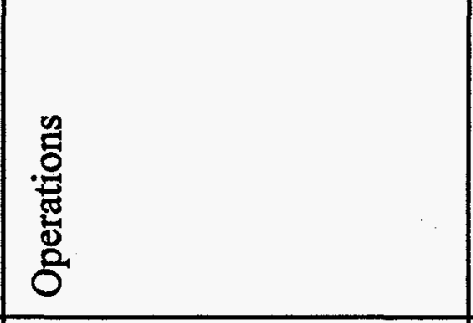 & 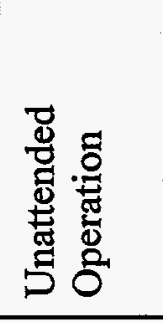 \\
\hline 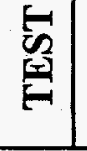 & $\stackrel{n}{\dot{1}}$ & $\overline{5}$ & 芒 & $\frac{m}{b}$ \\
\hline
\end{tabular}


TABLE 1 (Cont'd)

\begin{tabular}{|c|c|c|c|c|}
\hline TEST & NAME & OBJECTIVE & EXECUTION & RESULTS \\
\hline OT-4 & MTTR & $\begin{array}{l}\text { The system should } \\
\text { have modular } \\
\text { components which are } \\
\text { easily replaced or } \\
\text { repaired. Mean-time- } \\
\text { to-repair (MTTR) } \\
\text { should be less than } \\
72 \mathrm{~h} \text {. }\end{array}$ & $\begin{array}{l}\text { TOD: Review the system for ease } \\
\text { of maintenance. Report any } \\
\text { recommended changes to system } \\
\text { design. Report the MTTR. }\end{array}$ & $\begin{array}{l}\text { - TOD: The equipment meets the } \\
\text { requirement for MTTR. }\end{array}$ \\
\hline OT-5 & Service Interval & $\begin{array}{l}\text { Document all } \\
\text { preventative } \\
\text { maintenance performed } \\
\text { during the field test. }\end{array}$ & $\begin{array}{l}\text { EML: Report all system errors and } \\
\text { mechanical breakdowns not noted in } \\
\text { other test cases. Report any } \\
\text { maintenance (including } \\
\text { preventative) performed on the } \\
\text { system. } \\
\text { TOD: Report the calculated service } \\
\text { interval. }\end{array}$ & $\begin{array}{l}\text { EML: Unable to differentiate between } \\
\text { repairs and preventative maintenance. See } \\
\text { list of repairs under OT-3. } \\
\text { - TOD: Shortest interval of maintenance is } \\
\text { the main compressor ( } 5000 \mathrm{hrs.} \text {. All } \\
\text { adsorbents may be replaced at } 1 \text { year } \\
\text { intervals. If proper amount of nitrogen gas } \\
\text { is kept on hand, system can run for the } \\
\text { required 6-month service interval. }\end{array}$ \\
\hline OT-6 & $\begin{array}{l}\text { Restocking } \\
\text { Interval }\end{array}$ & $\begin{array}{l}\text { Determine the system } \\
\text { restocking interval. }\end{array}$ & $\begin{array}{l}\text { TOD: Inspect the system and any } \\
\text { related field test results. Determine } \\
\text { and report the unit restocking } \\
\text { interval. }\end{array}$ & $\begin{array}{l}\text { TOD: Restocking interval will be set by the } \\
\text { amount of nitrogen gas kept at each } \\
\text { location. Nitrogen is the lowest interval of } \\
\text { consumed material. Three bottles should } \\
\text { be kept on hand to have the restocking } \\
\text { interval set at 6-months. }\end{array}$ \\
\hline OT-7 & $\begin{array}{l}\text { System Mean- } \\
\text { time-Between- } \\
\text { Failure (MTBF) } \\
\text { should be at } \\
\text { least } 12 \text { months. }\end{array}$ & $\begin{array}{l}\text { Document any failures } \\
\text { observed during the } \\
\text { field test. }\end{array}$ & $\begin{array}{l}\text { TOD: Calculate the MTBF based } \\
\text { on available data. Make any } \\
\text { recommendations to improve } \\
\text { system MTBF. }\end{array}$ & $\begin{array}{l}\text { - TOD: System MTBF is calculated at } 1700 \\
\text { hours (70 days). }\end{array}$ \\
\hline
\end{tabular}


TABLE 1 (Cont'd)

\begin{tabular}{|c|c|c|c|c|}
\hline TEST & NAME & OBJECTIVE & EXECUTION & RESULTS \\
\hline OT-8 & Self-Diagnostics & $\begin{array}{l}\text { The system should } \\
\text { have self-diagnostics to } \\
\text { detect operational } \\
\text { degradation. }\end{array}$ & $\begin{array}{l}\text { Document operation of self- } \\
\text { diagnostics during the test period. }\end{array}$ & $\begin{array}{l}\text { Operator's interpretation of critical } \\
\text { parameters from State of Health Information } \\
\text { required. } \\
\text { - Automatic computer-generated notification } \\
\text { of specific malfunctions would be } \\
\text { desirable. } \\
\text { - Software modified after repair from } \\
\text { overheating. Log file listing all events } \\
\text { placed in operation. Highlighted line } \\
\text { indicates malfunction. }\end{array}$ \\
\hline OT-9 & $\begin{array}{l}\text { Commercial } \\
\text { Components }\end{array}$ & $\begin{array}{l}\text { The system should be } \\
\text { constructed with off- } \\
\text { the-shelf components, } \\
\text { where possible. }\end{array}$ & $\begin{array}{l}\text { TOD: Inspect the parts list and } \\
\text { report components which are not } \\
\text { off-the-shelf. }\end{array}$ & $\begin{array}{l}\text { TOD: A majority of parts bought from } \\
\text { commercial vendors. The final collection } \\
\text { trap, Ascarite trap and the counting system } \\
\text { sample chamber developed by PNNL. } \\
\text { Cryogenic valves modified by PNNL. }\end{array}$ \\
\hline OT-10 & $\begin{array}{l}\text { Sample Bottle } \\
\text { Pressure }\end{array}$ & $\begin{array}{l}\text { Sample bottle pressure } \\
\text { cannot exceed } 20 \text { psig. }\end{array}$ & $\begin{array}{l}\text { Measure sample bottle pressures for } \\
\text { five collective cycles. }\end{array}$ & $\begin{array}{l}\text { - Bottle pressure for } 6 \text { collection cycles } \\
\text { ranged from } 44 \text { to } 56 \text { Torr. }\end{array}$ \\
\hline OT-11 & $\begin{array}{l}\text { Power Outage } \\
\text { Recovery }\end{array}$ & $\begin{array}{l}\text { Demonstrate that the } \\
\text { system "reboots" itself } \\
\text { after power outages of } \\
5 \mathrm{sec}, 10 \mathrm{~min}, 3 \mathrm{~h} \text { and } \\
24 \mathrm{~h} \text {. }\end{array}$ & $\begin{array}{l}\text { Determine that data is not lost after } \\
\text { a power outage. Simulate power } \\
\text { outage while unit is in collection, } \\
\text { separation and counting modes (all } \\
\text { simultaneously running). }\end{array}$ & $\begin{array}{l}\text { Data not lost after power outage. } \\
\text { - Reboot tests after timed outages not } \\
\text { performed. }\end{array}$ \\
\hline PGT-1 & Size & $\begin{array}{l}\text { System dimensions } \\
\text { will not exceed 6' x } 3^{\prime} \text { x } \\
3^{\prime}\left(72^{\prime \prime} \text { x } 36^{\prime \prime} \text { x 36"). }\right.\end{array}$ & $\begin{array}{l}\text { TOD: Measure the overall size of } \\
\text { the system. }\end{array}$ & $\begin{array}{l}\text { - Normal dimensions are } 71 \text { " w x } 35^{\prime \prime} \mathrm{d} \text { x } \\
80 " \mathrm{~h} \text { (with front end closed). } \\
\text { - System does not meet size specification. }\end{array}$ \\
\hline
\end{tabular}


TABLE 1 (Cont'd)

\begin{tabular}{|c|c|c|c|c|}
\hline TEST & NAME & OBJECTIVE & EXECUTION & RESULTS \\
\hline PGT-2 & Weight & $\begin{array}{l}\text { System shall contain } \\
\text { modular components } \\
\text { that do not require } \\
\text { more than one person } \\
\text { to lift (40 lbs), except } \\
\text { during installation. } \\
\text { Total system weight } \\
\text { should not exceed } \\
1000 \mathrm{lb} \text {. }\end{array}$ & $\begin{array}{l}\text { TOD: Measure weights and } \\
\text { individual modules during system } \\
\text { assembly at EML. Determine the } \\
\text { weight of the total system by } \\
\text { summing weights of the individual } \\
\text { modules. }\end{array}$ & $\begin{array}{l}\text { - TOD: Total system weight is } 3404 \mathrm{lbs} \text {. } \\
\text { - The system does not meet the weight } \\
\text { requirements. }\end{array}$ \\
\hline PGT-3 & Noise & $\begin{array}{l}\text { The closed system } \\
\text { should operate at } 60 \\
\text { dBA or less at one } \\
\text { meter from each face } \\
\text { and corner of the unit } \\
\text { throughout unit } \\
\text { operation. }\end{array}$ & $\begin{array}{l}\text { TOD: During normal unit } \\
\text { operation, measure the continuous } \\
\text { system noise level as specified. } \\
\text { Measurements should be made of } \\
\text { any short-duration functions which } \\
\text { the test conductor deems to be } \\
\text { louder than continuous levels. }\end{array}$ & $\begin{array}{l}\text { - TOD: The system does not meet the noise } \\
\text { requirements. }\end{array}$ \\
\hline PGT-4 & $\begin{array}{l}\text { Temperature } \\
\text { Range }\end{array}$ & $\begin{array}{l}\text { The system should be } \\
\text { capable of operating } \\
\text { between } 50^{\circ} \text { to } 100^{\circ} \mathrm{F} \text {. }\end{array}$ & $\begin{array}{l}\text { Environmental Chamber not used. } \\
\text { Record the room temperature and } \\
\text { humidity at } 15 \text { minute intervals. For } \\
\text { higher temperatures allow room to } \\
\text { reach outside (summer) temperature } \\
\text { conditions. }\end{array}$ & $\begin{array}{l}\text { Unit subjected to room temperatures } \\
\text { ranging from } 65^{\circ} \mathrm{F} \text { to } 84^{\circ} \mathrm{F} \text {. No } \\
\text { malfunctions, damage to components or } \\
\text { changes in analytical functions attributed to } \\
\text { room temperature conditions. }\end{array}$ \\
\hline PGT-5 & $\begin{array}{l}\text { Shipping } \\
\text { Requirement }\end{array}$ & $\begin{array}{l}\text { There should be no } \\
\text { special shipping } \\
\text { requirements for the } \\
\text { system. There should } \\
\text { be no special } \\
\text { requirements for } \\
\text { shipping or storing } \\
\text { anticipated replacement } \\
\text { parts and materials. }\end{array}$ & $\begin{array}{l}\text { PNNL: Supply a written concept of } \\
\text { how the sampler and replacement } \\
\text { parts and materials will be shipped. } \\
\text { TOD: Report recommend changes, } \\
\text { if any, to the PNNL shipping } \\
\text { concept. }\end{array}$ & $\begin{array}{l}\text { - TOD: The system meets the requirements } \\
\text { for shipping. The Ascarite does not require } \\
\text { special shipping. }\end{array}$ \\
\hline
\end{tabular}




\begin{tabular}{|c|c|c|c|c|}
\hline $\begin{array}{l}0 \\
5 \\
5 \\
0 \\
0 \\
0 \\
2\end{array}$ & 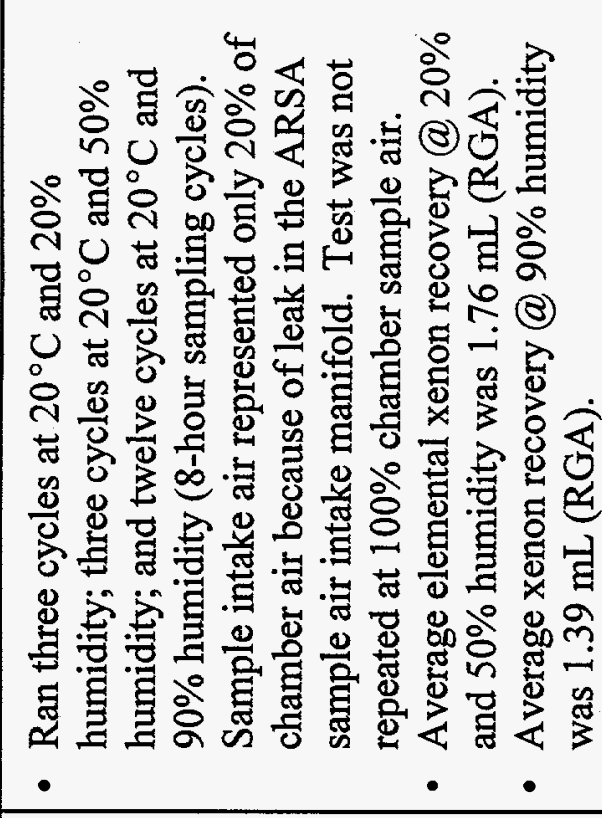 & 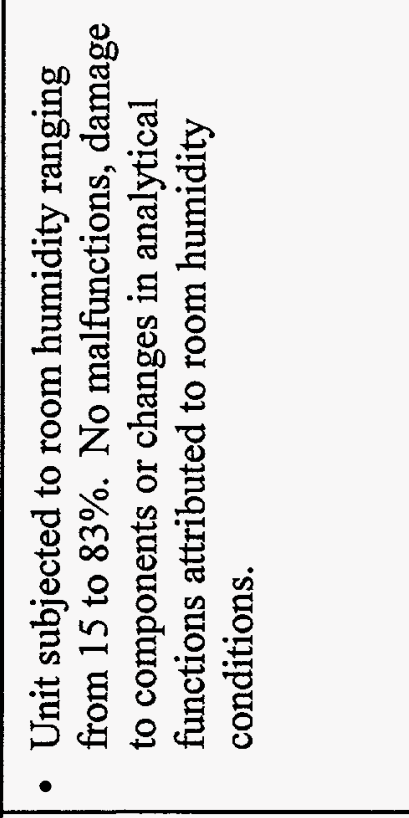 & 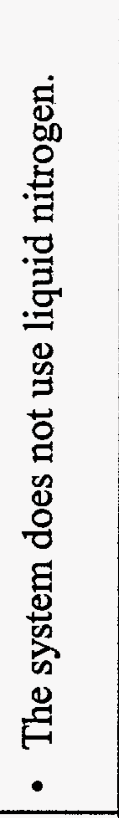 & 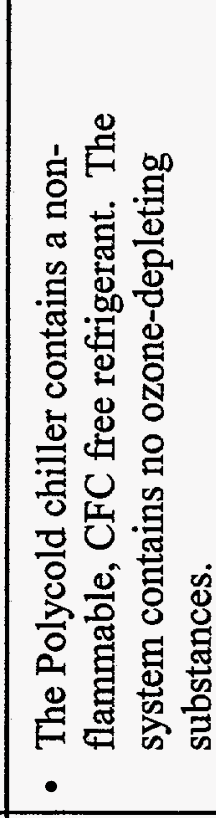 \\
\hline 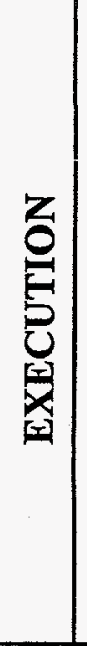 & 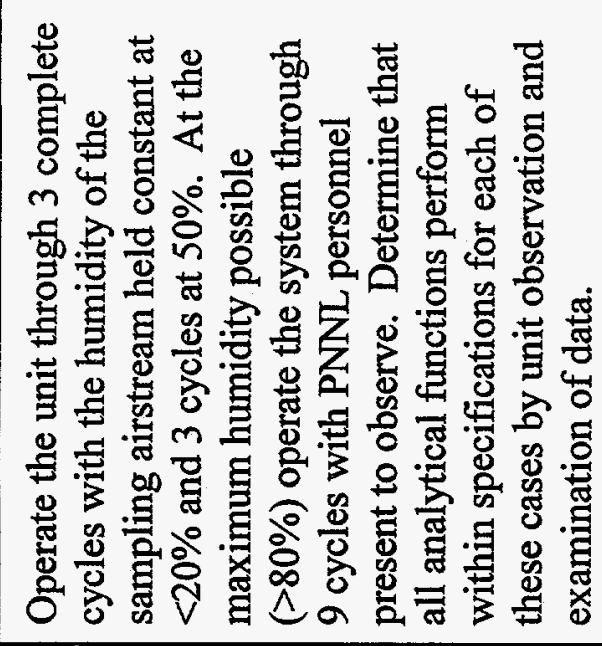 & 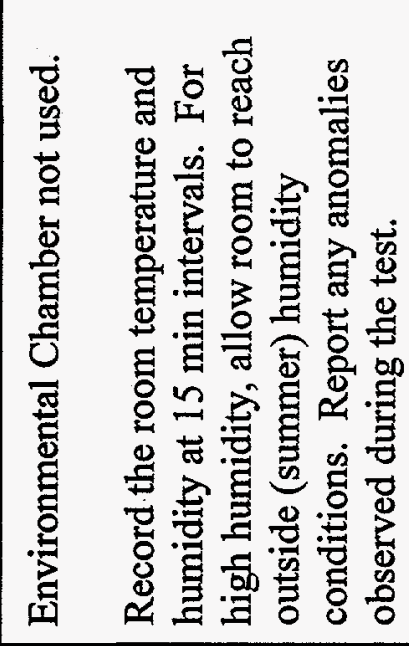 & 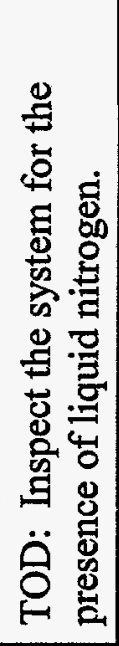 & 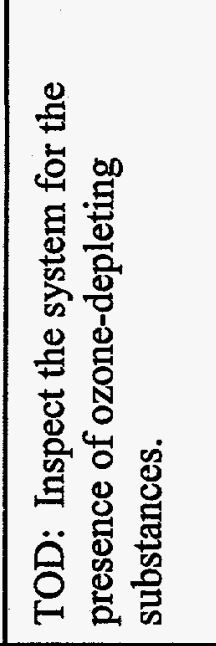 \\
\hline 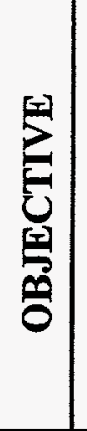 & 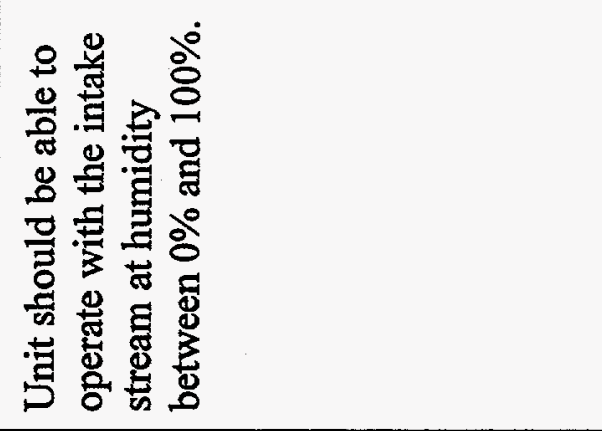 & 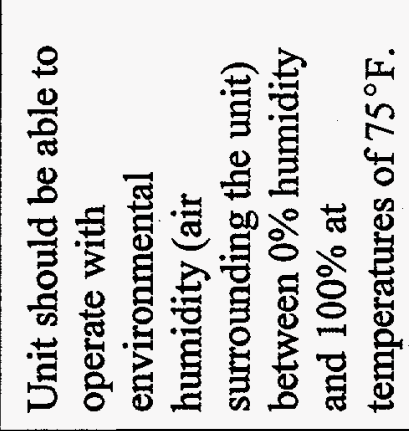 & 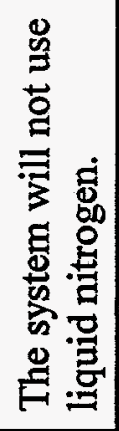 & 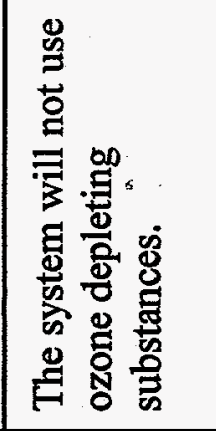 \\
\hline 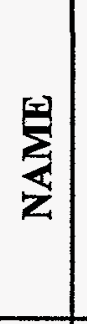 & 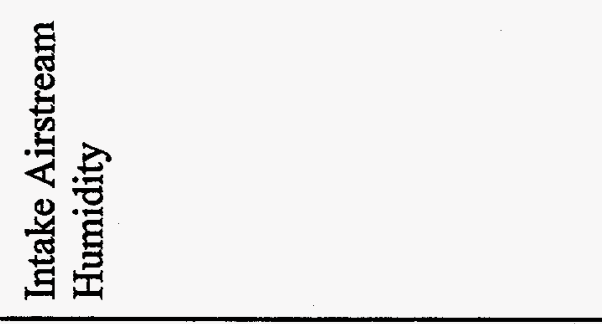 & 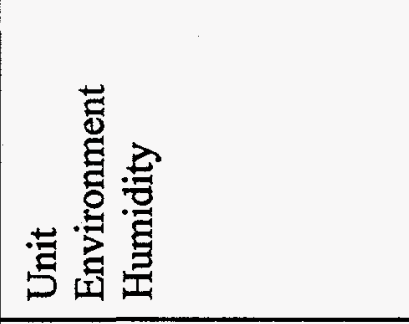 & 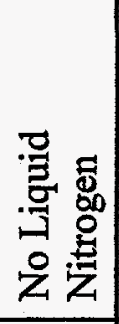 & 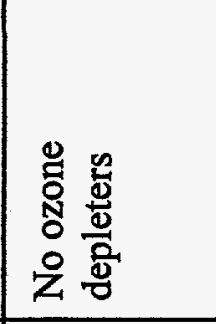 \\
\hline 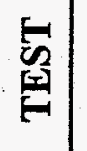 & 总 & 䓌 & 罚 & 站 \\
\hline
\end{tabular}


TABLE 1 (Cont'd)

\begin{tabular}{|c|c|c|c|c|c|}
\hline TEST & NAME & OBJECTIVE & EXECUTION & \multicolumn{2}{|l|}{ RESULTS } \\
\hline PGT-10 & Operation cost & $\begin{array}{l}\text { The annual operation } \\
\text { cost, additional } \\
\text { materials and supplies, } \\
\text { technician time, and } \\
\text { number of visits/year } \\
\text { should be estimated for } \\
\text { the system. }\end{array}$ & $\begin{array}{l}\text { TOD: Request available } \\
\text { information from PNNL. Review } \\
\text { EML daily logs and make } \\
\text { observations as necessary to } \\
\text { complete determination. Report } \\
\text { annual material requirements and } \\
\text { costs, mean-time-between-failure, } \\
\text { mean-time-to-repair, and significant } \\
\text { maintenance issues observed. }\end{array}$ & $\begin{array}{l}\text { TOD: The cost analysis is } \\
\text { technician visiting the site } \\
\text { - Annual Operating Cost: } \\
\text { 1) Power } \\
\text { 2) Consumables } \\
\text { 3) Technician Travel } \\
\text { (4.1 times/year) } \\
\text { Projected Total Annual Cost }\end{array}$ & $\begin{array}{l}\text { d on a } \\
\text { e a year. } \\
\begin{array}{r}3400.00 \\
1624.00 \\
20,500.00 \\
\$ 25,524.00\end{array}\end{array}$ \\
\hline PGT-11 & $\begin{array}{l}\text { Calibration } \\
\text { Source }\end{array}$ & $\begin{array}{l}\text { Internal calibration } \\
\text { sources should be } \\
\text { small enough that } \\
\text { special } \\
\text { transportation/handling } \\
\text { requirements and site } \\
\text { licenses are not needed } \\
\text { to ship and operate the } \\
\text { unit. }\end{array}$ & $\begin{array}{l}\text { PNNL: Document calibration } \\
\text { sources. } \\
\text { TOD: Review PNNL } \\
\text { documentation and determine any } \\
\text { special shipping or handling } \\
\text { requirements. Report any shipping } \\
\text { or operating requirements. }\end{array}$ & $\begin{array}{l}\text { - The system calibration sou } \\
\text { activity of } 0.5 \mathrm{nCi} \text { each. } \\
\text { - No special license needed }\end{array}$ & $\begin{array}{l}\text { have an } \\
\text { hipment. }\end{array}$ \\
\hline
\end{tabular}




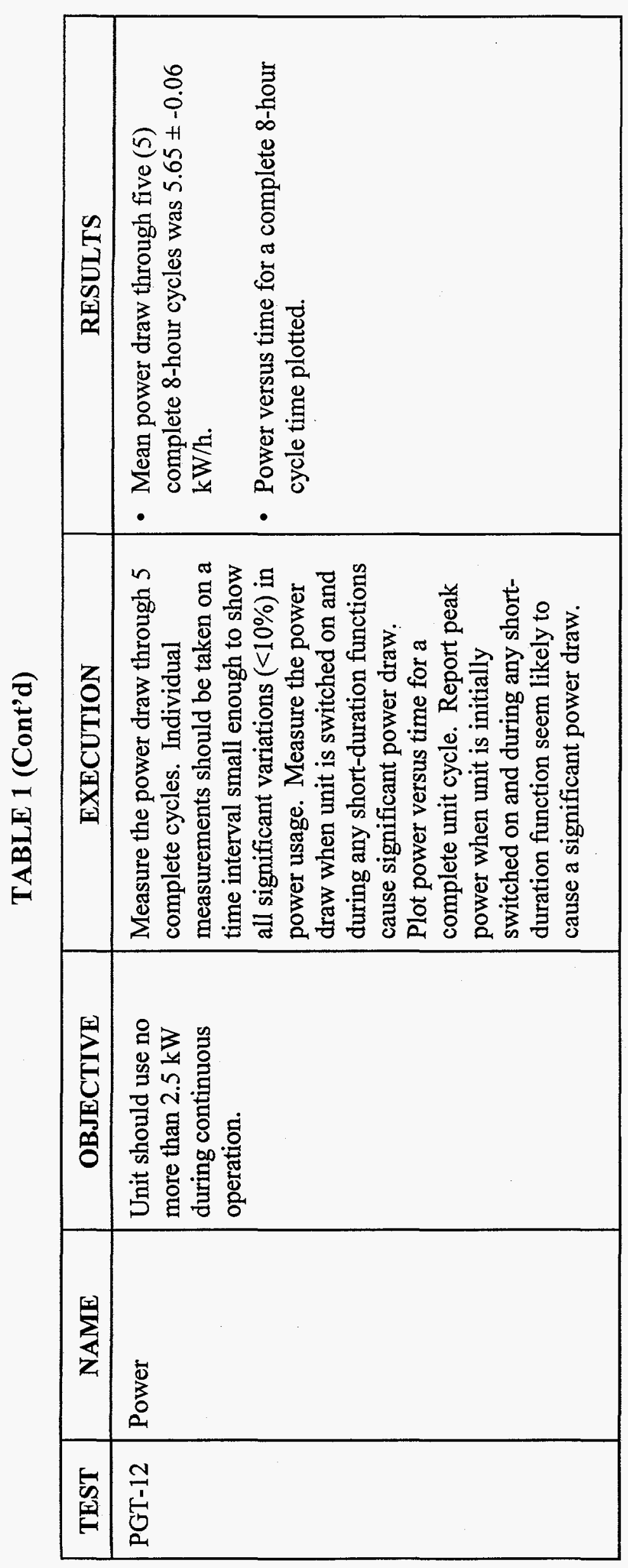


TABLE 2

RESULTS OF ARSA SAMPLING AND ANALYSIS

SITE NUMBER: $805 \mathrm{X}$

\begin{tabular}{|c|c|c|c|c|c|c|c|c|c|c|c|c|c|c|}
\hline $\begin{array}{c}\text { Sample } \\
\text { Date }\end{array}$ & $\begin{array}{c}\mathrm{J} \\
\text { Date }\end{array}$ & $\begin{array}{l}\text { Clock } \\
\text { time }\end{array}$ & $\begin{array}{l}\text { Sample } \\
\text { Dux. } \\
\text { (Min) }\end{array}$ & $\begin{array}{l}\text { Flow } \\
\text { Rate } \\
\text { (L/Min) }\end{array}$ & $\begin{array}{l}\text { Samp. } \\
\text { Vol. } \\
\text { (SCM) }\end{array}$ & $\begin{array}{l}\text { Det. } \\
\text { Cell } \\
\text { No. }\end{array}$ & $\begin{array}{c}\mathrm{Xe}-133 \\
(\mathrm{~B}-\mathrm{G}) \\
(\mathrm{pCi} / \mathrm{SCM})\end{array}$ & $\begin{array}{l}\text { Coll. } \\
\text { Bot. } \\
\text { No. }\end{array}$ & $\begin{array}{c}\mathrm{Xe}-133 \\
(\text { Anal }) \\
(\mathrm{pCi} / \mathrm{SCM})\end{array}$ & $\begin{array}{c}\text { Xe } \\
\text { Recov. } \\
\text { (Anal) } \\
\text { (mL) }\end{array}$ & $\begin{array}{c}\text { Xe } \\
\text { Recov. } \\
\text { (RGA) } \\
(\mathrm{mL})\end{array}$ & $\begin{array}{c}\text { Exp } \\
\text { Total } \\
\text { Xe } \\
(\mathrm{mL})\end{array}$ & $\begin{array}{c}\text { Xe } \\
\text { Recov } \\
\text { (Anal.) } \\
\left(\frac{\circ}{0}\right)\end{array}$ & $\begin{array}{l}\text { Flag } \\
\text { No. }\end{array}$ \\
\hline 8-Apr-97 & 098 & 1006 & 485 & 95.0 & 46.08 & & 0.000 & 5001 & 0.020 & 0.70 & 0.00 & 4.01 & 17.5 & \\
\hline 8-Apr-97 & 098 & 1811 & 530 & 95.0 & 50.35 & & 0.000 & 0317 & 0.005 & 0.70 & 0.00 & 4.38 & 16.0 & \\
\hline 9-Apr-97 & 099 & 0301 & 485 & 95.0 & 46.08 & & 0.000 & 7113 & 0.006 & 0.60 & 0.00 & 4.01 & 15.0 & \\
\hline $21-A p r-97$ & 111 & 1000 & 480 & 95.0 & 45.60 & 4 & 0.089 & 4472 & 0.045 & 0.64 & 0.02 & 3.97 & 16.1 & \\
\hline 21-Apr-97 & 111 & 1800 & 480 & 95.0 & 45.60 & 1 & 0.132 & 2902 & 0.046 & 0.62 & 0.01 & 3.97 & 15.6 & \\
\hline $22-A p r-97$ & 112 & 0200 & 480 & 95.0 & 45.60 & 2 & 0.248 & 1994 & 0.656 & 0.62 & 0.13 & 3.97 & 15.6 & \\
\hline 22-Apr-97 & 112 & 1000 & 480 & 95.0 & 45.60 & 3 & 0.089 & 0998 & 0.181 & 0.59 & 0.16 & 3.97 & 14.9 & \\
\hline 22-Apr-97 & 112 & 1800 & 480 & 95.0 & 45.60 & 4 & $0.132^{\prime}$ & 2560 & 0.304 & 0.66 & 0.10 & 3.97 & 16.6 & \\
\hline $23-A p r-97$ & 113 & 0200 & 480 & 95.0 & 45.60 & 1 & 0.000 & $\mathrm{E} 661$ & 0.063 & 0.30 & 0.30 & $3: 97$ & 7.6 & \\
\hline 23-Apr-97 & 113 & 1000 & 480 & 95.0 & 45.60 & 2 & 0.000 & 2436 & 0.095 & 0.70 & 0.17 & 3.97 & 17.6 & 2 \\
\hline 23-Apr-97 & 113 & 1737 & 480 & 95.0 & 45.60 & 4 & 0.205 & & 0.000 & 0.00 & 0.00 & 3.97 & & 1 \\
\hline 24-Apr-97 & 114 & 0136 & 480 & 95.0 & 45.60 & 1 & 0.062 & & 0.000 & 0.00 & 0.00 & 3.97 & & 1 \\
\hline 26-Apr-97 & 116 & 0712 & 480 & 95.0 & 45.60 & 4 & 0.338 & & 0.000 & 0.00 & 0.00 & 3.97 & & 1 \\
\hline 26-Apr-97 & 116 & 1513 & 480 & 95.0 & 45.60 & 1 & 0.586 & & 0.000 & 0.00 & 0.00 & 3.97 & & 1 \\
\hline 28-Apr-97 & 118 & 0208 & 480 & 95.0 & 45.60 & 4 & 0.070 & & 0.000 & 0.00 & 0.00 & 3.97 & & 1 \\
\hline 28-Apr-97 & 118 & 1832 & 480 & 95.0 & 45.60 & 2 & 0.329 & & 0.000 & 0.00 & 0.00 & 3.97 & & 1 \\
\hline 30-Apr-97 & 120 & 0300 & 480 & 95.0 & 45.60 & 2 & 0.000 & 2839 & 0.785 & 0.45 & 0.18 & 3.97 & 11.3 & 3 \\
\hline 30-Apr-97 & 120 & 1100 & 480 & 95.0 & 45.60 & 3 & 1.274 & 0737 & 0.896 & 0.75 & 0.18 & 3.97 & 18.9 & 3 \\
\hline
\end{tabular}


TABLE 2 (Cont'd)

RESULTS OF ARSA SAMPLING AND ANALYSIS

SITE NUMBER: $805 \mathrm{X}$

Sample J Clock Sample Flow Samp. Det. Xe-133 Coll. Xe-133 Xe Xe Exp Xe Date Date time Dur. Rate Vol. Cell (B-G) Bot. (Anal) Recov. Recov. Total Recov No.

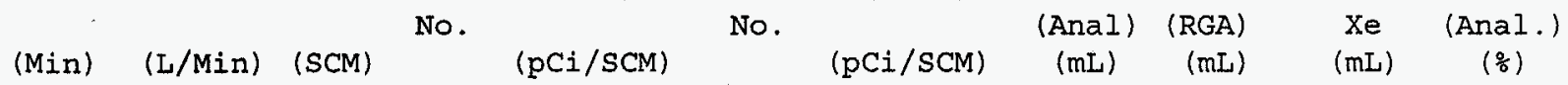

\begin{tabular}{|c|c|c|c|c|c|c|c|c|c|c|c|c|c|}
\hline $30-A p r-97$ & 120 & 1900 & 480 & 95.0 & 45.60 & 4 & 0.734 & 3989 & 0.737 & 0.53 & 0.21 & 3.97 & 13.4 \\
\hline 1-May-97 & 121 & 0300 & 480 & 95.0 & 45.60 & 1 & 0.416 & 0694 & 0.582 & 0.81 & 0.18 & 3.97 & 20.4 \\
\hline 1-May-97 & 121 & 1100 & 480 & 95.0 & 45.60 & 2 & 0.486 & 4290 & 0.253 & 0.75 & 0.06 & 3.97 & 18.9 \\
\hline 4 -Aug - 97 & 216 & 1230 & 810 & 100.0 & 81.00 & & 0.000 & 1472 & 0.000 & 0.28 & 0.00 & 7.05 & 4.0 \\
\hline 4-Aug-97 & 217 & 0200 & 480 & 100.0 & 48.00 & 3 & 0.000 & 1474 & 0.000 & 0.23 & 0.00 & 4.18 & 5.5 \\
\hline 5-Aug-97 & 217 & 1000 & 480 & 100.0 & 48.00 & 4 & 0.000 & 4438 & 0.000 & 0.20 & 0.50 & 4.18 & 4.8 \\
\hline 5-Aug- 97 & 217 & 1800 & 480 & 100.0 & 48.00 & 1 & 0.000 & 1817 & 0.361 & 0.36 & 0.10 & 4.18 & 8.6 \\
\hline 6-Aug- 97 & 218 & 0200 & 480 & 100.0 & 48.00 & 2 & 0.000 & 3161 & 0.402 & 0.61 & 0.10 & 4.18 & 14.6 \\
\hline 6-Aug- 97 & 218 & 1000 & 480 & 100.0 & 48.00 & 3 & 0.000 & 4841 & 0.310 & 0.78 & 0.21 & 4.18 & 18.7 \\
\hline 6-Aug-97 & 218 & 1800 & 480 & 100.0 & 48.00 & 4 & 0.000 & 2525 & 0.247 & 0.85 & 0.30 & 4.18 & 20.4 \\
\hline 7-Aug-97 & 219 & 0200 & 480 & 100.0 & 48.00 & 1 & 0.000 & 0731 & 0.320 & 0.86 & 0.00 & 4.18 & 20.6 \\
\hline 7-Aug- 97 & 219 & 1000 & 480 & 100.0 & 48.00 & 2 & 0.000 & 0284 & 1.320 & 1.35 & 0.00 & 4.18 & 32.3 \\
\hline 7-Aug-97 & 219 & 1800 & 480 & 100.0 & 48.00 & 3 & 0.000 & 1406 & 1.020 & 1.34 & 0.40 & 4.18 & 32.1 \\
\hline 8-Aug- 97 & 220 & 0200 & 480 & 100.0 & 48.00 & 4 & 0.000 & 3703 & 0.910 & 1.18 & 0.50 & 4.18 & 28.3 \\
\hline 15-Aug- 97 & 227 & 0200 & 480 & 100.0 & 48.00 & 2 & 1.360 & 1640 & 1.250 & 0.32 & 0.70 & 4.18 & 7.7 \\
\hline 15-Aug-97 & 227 & 1000 & 480 & 100.0 & 48.00 & 3 & 1.720 & 7836 & 1.385 & 0.17 & 0.30 & 4.18 & 4.1 \\
\hline 16-Aug- 97 & 228 & 0200 & 480 & 100.0 & 48.00 & 1 & 0.268 & 1056 & 0.175 & 0.15 & 0.20 & 4.18 & 3.6 \\
\hline $16-$ Aug -97 & 228 & 1000 & 480 & 100.0 & 48.00 & 2 & 0.175 & 1508 & 0.078 & 0.17 & 0.40 & 4.18 & 4.1 \\
\hline $16-$ Aug - 97 & 228 & 1800 & 480 & 100.0 & 48.00 & 3 & 0.099 & 0177 & 0.065 & 0.12 & 0.20 & 4.18 & 2.9 \\
\hline
\end{tabular}


TABLE 2 (Cont'd)

RESULTS OF ARSA SAMPLING AND ANALYSIS

SITE NUMBER : $805 \mathrm{X}$

\begin{tabular}{|c|c|c|c|c|c|c|c|c|c|c|c|c|c|c|}
\hline $\begin{array}{c}\text { Sample } \\
\text { Date }\end{array}$ & $\begin{array}{c}\text { J } \\
\text { Date }\end{array}$ & $\begin{array}{l}\text { Clock } \\
\text { time }\end{array}$ & $\begin{array}{l}\text { Sample } \\
\text { Dur. } \\
\text { (Min) }\end{array}$ & $\begin{array}{l}\text { Flow } \\
\text { Rate } \\
\text { (L/Min) }\end{array}$ & $\begin{array}{l}\text { Samp. } \\
\text { Vol. } \\
\text { (SCM) }\end{array}$ & $\begin{array}{l}\text { Det. } \\
\text { Celi } \\
\text { No. }\end{array}$ & $\begin{array}{c}\mathrm{Xe}-133 \\
(\mathrm{~B}-\mathrm{G}) \\
(\mathrm{pCi} / \mathrm{SCM})\end{array}$ & $\begin{array}{l}\text { Col1. } \\
\text { Bot. } \\
\text { No. }\end{array}$ & $\begin{array}{r}\mathrm{Xe}-133 \\
\text { (Anal) } \\
\text { (pCi/SCM) }\end{array}$ & $\begin{array}{c}\text { Xe } \\
\text { Recov. } \\
\text { (Anal) } \\
\text { (mL) }\end{array}$ & $\begin{array}{c}\text { Xe } \\
\text { Recov. } \\
(R G A) \\
(m L)\end{array}$ & $\begin{array}{c}\operatorname{Exp} \\
\text { Total } \\
\text { Xe } \\
(\mathrm{mL})\end{array}$ & $\begin{array}{c}\text { Xe } \\
\text { Recov } \\
\text { (Anal.) } \\
\left(\frac{\%}{6}\right)\end{array}$ & $\begin{array}{l}\text { Flag } \\
\text { No. }\end{array}$ \\
\hline 17-Aug-97 & 229 & 0200 & 480 & 100.0 & 48.00 & 4 & 0.122 & 0346 & 0.015 & 0.13 & 0.20 & 4.18 & 3.1 & \\
\hline 17-Aug-97 & 229 & 1000 & 480 & 100.0 & 48.00 & 2 & 0.016 & 2075 & 0.005 & 0.14 & 0.20 & 4.18 & 3.4 & \\
\hline 17-Aug-97 & 229 & 1800 & 480 & 100.0 & 48.00 & 2 & 0.000 & 5361 & 0.000 & 0.13 & 0.20 & 4.18 & 3.1 & 12 \\
\hline 18-Aug-97 & 230 & 0200 & 480 & 100.0 & 48.00 & 3 & 0.263 & 1698 & 0.286 & 0.18 & 0.30 & 4.18 & 4.3 & \\
\hline 18 -Aug- 97 & 230 & 1000 & 480 & 100.0 & 48.00 & 4 & 0.269 & F130 & 0.153 & 1.12 & 1.70 & 4.18 & 26.8 & 5 \\
\hline 18 -Aug- 97 & 230 & 1800 & 480 & 100.0 & 48.00 & 1 & 0.202 & 0660 & 0.197 & 1.29 & 1.90 & 4.18 & 30.9 & 5 \\
\hline 19-Aug-97 & 231 & 0200 & 480 & 100.0 & 48.00 & 2 & 0.000 & $\mathrm{E} 468$ & 0.896 & 0.19 & 0.40 & 4.18 & 4.5 & 5 \\
\hline 19-Aug-97 & 231 & 1000 & 480 & 100.0 & 48.00 & 3 & 0.741 & 1138 & 0.390 & 1.20 & 1.70 & 4.18 & 28.7 & 6 \\
\hline 19-Aug-97 & 231 & 1800 & 480 & 100.0 & 48.00 & 4 & 0.561 & 4091 & 0.363 & 1.13 & 1.80 & 4.18 & 27.1 & 6 \\
\hline 20-Aug-97 & 232 & 0200 & 480 & 100.0 & 48.00 & 1 & 0.514 & F598 & 0.437 & 1.29 & 1.70 & 4.18 & 30.9 & 6 \\
\hline 20-Aug- 97 & 232 & 1000 & 480 & 100.0 & 48.00 & 2 & 0.271 & 1817 & 0.186 & 1.17 & 1.90 & 4.18 & 28.0 & 7 \\
\hline 20-Aug- 97 & 232 & 1800 & 480 & 100.0 & 48.00 & 3 & 0.105 & 3703 & 0.060 & 1.02 & 1.90 & 4.18 & 24.4 & 7 \\
\hline 21 -Aug - 97 & 233 & 0200 & 480 & 100.0 & 48.00 & 4 & 0.085 & E831 & 0.079 & 0.65 & 1.10 & 4.18 & 15.6 & 7 \\
\hline 21-Aug-97 & 233 & 1000 & 480 & 100.0 & 48.00 & 1 & 0.076 & 3161 & 0.085 & 0.71 & 1.20 & 4.18 & 17.0 & 7 \\
\hline 21-Aug-97 & 233 & 1800 & 480 & 100.0 & 48.00 & 2 & 0.269 & 2186 & 0.229 & 0.81 & 1.30 & 4.18 & 19.4 & 7 \\
\hline 22-Aug-97 & 234 & 0200 & 480 & 100.0 & 48.00 & 3 & 0.053 & 2947 & 0.046 & 0.73 & 1.30 & 4.18 & 17.5 & 7 \\
\hline $22-A u g-97$ & 234 & 1000 & 480 & 100.0 & 48.00 & 4 & 0.142 & 0731 & 0.173 & 0.10 & 0.00 & 4.18 & 2.4 & 7 \\
\hline $22-A u g-97$ & 234 & 1800 & 480 & 100.0 & 48.00 & 1 & 0.236 & 0153 & 0.255 & 0.71 & 1.30 & 4.18 & 17.0 & 7 \\
\hline 23-Aug-97 & 235 & 0200 & 480 & 100.0 & 48.00 & 2 & 0.566 & 2525 & 0.518 & 0.77 & 1.30 & 4.18 & 18.4 & 7 \\
\hline
\end{tabular}


TABLE 2 (Cont'd)

RESULTS OF ARSA SAMPLING AND ANALYSIS

SITE NUMBER; $805 \mathrm{X}$

\begin{tabular}{|c|c|c|c|c|c|c|c|c|c|c|c|c|c|c|}
\hline $\begin{array}{c}\text { Sample } \\
\text { Date }\end{array}$ & $\begin{array}{c}\text { J } \\
\text { Date }\end{array}$ & $\begin{array}{l}\text { Clock } \\
\text { time }\end{array}$ & $\begin{array}{l}\text { Sample } \\
\text { Dux. } \\
\text { (Min) }\end{array}$ & $\begin{array}{l}\text { Flow } \\
\text { Rate } \\
\text { (L/Min) }\end{array}$ & $\begin{array}{l}\text { Samp. } \\
\text { Vol. } \\
\text { (SCM) }\end{array}$ & $\begin{array}{l}\text { Det. } \\
\text { Celi } \\
\text { No. }\end{array}$ & $\begin{array}{c}\mathrm{Xe}-133 \\
(\mathrm{~B}-\mathrm{G}) \\
(\mathrm{pCi} / \mathrm{SCM})\end{array}$ & $\begin{array}{c}\text { Coll. } \\
\text { Bot. } \\
\text { No. }\end{array}$ & $\begin{array}{c}\mathrm{Xe}-133 \\
(\mathrm{Anal}) \\
(\mathrm{pCi} / \mathrm{sCM})\end{array}$ & $\begin{array}{c}\text { Xe } \\
\text { Recov. } \\
\text { (Anal) } \\
\text { (mL) }\end{array}$ & $\begin{array}{c}\text { Xe } \\
\text { Recov. } \\
\text { (RGA) } \\
\text { (mL) }\end{array}$ & $\begin{array}{c}\text { Exp } \\
\text { Total } \\
\text { Xe } \\
(\mathrm{mL})\end{array}$ & $\begin{array}{c}\text { Xe } \\
\text { Recov } \\
\text { (Anal.) } \\
\left(\frac{9}{8}\right)\end{array}$ & $\begin{array}{c}\text { Flag } \\
\text { No. }\end{array}$ \\
\hline 23-Aug-97 & 235 & 1000 & 480 & 100.0 & 48.00 & 3 & 0.934 & 1081 & 0.701 & 0.91 & 1.40 & 4.18 & 21.8 & 7 \\
\hline 23-Aug-97 & 235 & 1800 & 480 & 100.0 & 48.00 & 4 & 0.392 & 1256 & 0.291 & 0.74 & 1.20 & 4.18 & 17.7 & 7 \\
\hline 24-Aug- 97 & 236 & 0200 & 480 & 100.0 & 48.00 & 1 & 0.057 & 1176 & 0.046 & 0.83 & 1.40 & 4.18 & 19.9 & 7 \\
\hline 24-Aug - 97 & 236 & 1000 & 480 & 100.0 & 48.00 & 2 & 0.103 & 0955 & 0.093 & 0.83 & 1.30 & 4.18 & 19.9 & \\
\hline 24-Aug-97 & 236 & 1800 & 480 & 100.0 & 48.00 & 3 & 0.069 & 0348 & 0.059 & 0.78 & 1.30 & 4.18 & 18.7 & \\
\hline 25-Aug- 97 & 237 & 0200 & 480 & 100.0 & 48.00 & 4 & 0.084 & 1640 & 0.066 & 0.82 & 1.30 & 4.18 & 19.6 & \\
\hline 25-Aug- 97 & 237 & 1000 & 480 & 100.0 & 48.00 & 1 & 0.102 & 0177 & 0.089 & 0.84 & 1.30 & 4.18 & 20.1 & \\
\hline 25-Aug - 97 & 237 & 1800 & 480 & 100.0 & 48.00 & 2 & 0.222 & 1056 & 0.107 & 0.11 & 0.00 & 4.18 & 2.6 & \\
\hline 26-Aug-97 & 238 & 0200 & 480 & 100.0 & 48.00 & 3 & 0.078 & 7852 & 0.055 & 0.90 & 1.40 & 4.18 & 21.6 & \\
\hline 26-Aug-97 & 238 & 1000 & 480 & 100.0 & 48.00 & 4 & 0.078 & 4073 & 0.161 & 0.70 & 1.10 & 4.18 & 16.8 & \\
\hline $26-$ Aug - 97 & 238 & 1800 & 480 & 100.0 & 48.00 & 1 & 0.611 & 4841 & 0.466 & 0.59 & 1.00 & 4.18 & 14.1 & \\
\hline 27-Aug-97 & 239 & 0200 & 480 & 100.0 & 48.00 & 2 & 0.323 & 0284 & 0.259 & 0.59 & 1.40 & 4.18 & 14.1 & 9 \\
\hline 27-Aug- 97 & 239 & 1000 & 480 & 100.0 & 48.00 & 3 & 0.463 & 1406 & 0.406 & 0.72 & 1.30 & 4.18 & 17.2 & \\
\hline 27-Aug-97 & 239 & 1800 & 480 & 100.0 & 48.00 & 4 & 0.373 & 2057 & 0.288 & 0.87 & 1.30 & 4.18 & 20.8 & \\
\hline 29-Aug-97 & 241 & 1000 & 480 & 100.0 & $48: 00$ & 2 & 0.150 & 1632 & 0.110 & 0.89 & 1.40 & 4.18 & 21.3 & \\
\hline $29-A u g-97$ & 241 & 1800 & 480 & 100.0 & 48.00 & 3 & 0.246 & F418 & 0.182 & 0.96 & 1.50 & 4.18 & 23.0 & \\
\hline $30-$ Aug - 97 & 242 & 0200 & 480 & 100.0 & 48.00 & 4 & 3.930 & F156 & 2.911 & 0.88 & 1.30 & 4.18 & 21.1 & \\
\hline 30-Aug-97 & 242 & 1000 & 480 & 100.0 & 48.00 & 1 & 1.341 & 2631 & 1.075 & 0.92 & 1.70 & 4.18 & 22.0 & \\
\hline 30-Aug-97 & 242 & 1800 & 480 & 100.0 & 48.00 & 2 & 1.726 & 1719 & 1.271 & 1.05 & 1.20 & 4.18 & 25.1 & \\
\hline
\end{tabular}


TABLE 2 (Cont'd)

RESULTS OF ARSA SAMPLING AND ANALYSIS

SITE NUMBER: $805 \mathrm{X}$

\begin{tabular}{|c|c|c|c|c|c|c|c|c|c|c|c|c|c|c|}
\hline $\begin{array}{c}\text { Sample } \\
\text { Date }\end{array}$ & $\begin{array}{c}\text { J } \\
\text { Date }\end{array}$ & $\begin{array}{l}\text { Clock } \\
\text { time }\end{array}$ & $\begin{array}{l}\text { Sample } \\
\text { Dur. } \\
\text { (Min) }\end{array}$ & $\begin{array}{l}\text { Flow } \\
\text { Rate } \\
\text { (L/Min) }\end{array}$ & $\begin{array}{l}\text { Samp. } \\
\text { Vol. } \\
\text { (SCM) }\end{array}$ & $\begin{array}{l}\text { Det. } \\
\text { Celi } \\
\text { No. }\end{array}$ & $\begin{array}{c}\mathrm{Xe}-133 \\
(\mathrm{~B}-\mathrm{G}) \\
(\mathrm{pCi} / \mathrm{SCM})\end{array}$ & $\begin{array}{l}\text { Coll. } \\
\text { Bot. } \\
\text { No. }\end{array}$ & $\begin{array}{c}\mathrm{Xe}-133 \\
(\mathrm{Anal}) \\
(\mathrm{pCi} / \mathrm{SCM})\end{array}$ & $\begin{array}{c}\text { Xe } \\
\text { Recov. } \\
\text { (Anal) } \\
\text { (mL) }\end{array}$ & $\begin{array}{c}\text { Xe } \\
\text { Recov. } \\
(\text { RGA }) \\
(\mathrm{mL})\end{array}$ & $\begin{array}{c}\operatorname{Exp} \\
\text { Total } \\
\text { Xe } \\
(\mathrm{mL})\end{array}$ & $\begin{array}{c}\text { Xe } \\
\text { Recov } \\
\text { (AnaI.) } \\
\left(\frac{\circ}{6}\right)\end{array}$ & $\begin{array}{l}\text { Flag } \\
\text { No. }\end{array}$ \\
\hline $1-$ Aug-97 & 243 & 0200 & 480 & 100.0 & 48.00 & 3 & 1.594 & E543 & 0.842 & 0.90 & 1.60 & 4.18 & 21.6 & \\
\hline 1 -Aug-97 & 243 & 1000 & 480 & 100.0 & 48.00 & 4 & 1.058 & 6101 & 1.059 & 0.93 & 1.40 & 4.18 & 22.3 & \\
\hline 1 -Aug-97 & 243 & 1800 & 480 & 100.0 & 48.00 & 1 & 0.964 & 0696 & 1.103 & 1.05 & 1.60 & 4.18 & 25.1 & \\
\hline 1-Sep-97 & 244 & 0200 & 480 & 100.0 & 48.00 & 2 & 0.745 & 2247 & 0.470 & 1.03 & 1.80 & 4.18 & 24.7 & \\
\hline $1-\operatorname{sep}-97$ & 244 & 1000 & 480 & 100.0 & 48.00 & 3 & 0.608 & 2094 & 0.616 & 0.90 & 1.50 & 4.18 & 21.6 & \\
\hline 1-Sep-97 & 244 & 1800 & 480 & 100.0 & 48.00 & 4 & 0.410 & 2371 & 0.330 & 0.89 & 1.40 & 4.18 & 21.3 & \\
\hline $2-\operatorname{sep}-97$ & 245 & 0200 & 480 & 100.0 & 48.00 & 1 & 0.161 & 51.74 & 0.195 & 1.07 & 1.70 & 4.18 & 25.6 & \\
\hline $2-\operatorname{sep}-97$ & 245 & 1000 & 480 & 100.0 & 48.00 & 2 & 0.167 & 2003 & 0.122 & 1.01 & 1.50 & 4.18 & 24.2 & \\
\hline $2-\operatorname{sep}-97$ & 245 & 1800 & 480 & 100.0 & 48.00 & 3 & 0.144 & 7836 & 0.000 & 0.00 & 1.70 & 4.18 & & 12 \\
\hline 3-Sep-97 & 246 & 0200 & 480 & 100.0 & 48.00 & 4 & 2.797 & E831 & 2.437 & 0.64 & 1.10 & 4.18 & 15.3 & \\
\hline 3-Sep- 97 & 246 & 1000 & 480 & 100.0 & 48.00 & 1 & 5.091 & 2075 & 5.250 & 0.53 & 1.00 & 4.18 & 12.7 & 9 \\
\hline 3-Sep- 97 & 246 & 1800 & 480 & 100.0 & 48.00 & 2 & 7.334 & 0660 & 5.930 & 0.87 & 1.40 & 4.18 & 20.8 & 9 \\
\hline $4-$ Sep- 97 & 247 & 0200 & 480 & 100.0 & 48.00 & 3 & 7.397 & 8938 & 7.850 & 0.59 & 1.10 & 4.18 & 14.1 & 9 \\
\hline $4-$ Sep- 97 & 247 & 1000 & 480 & 100.0 & 48.00 & 4 & 10.660 & 0797 & 9.170 & 0.69 & 1.10 & 4.18 & 16.5 & 9 \\
\hline 4-Sep- 97 & 247 & 1800 & 480 & 100.0 & 48.00 & 1 & 7.190 & 3703 & 8.639 & 0.74 & 1.30 & 4.18 & 17.7 & 9 \\
\hline $5-\operatorname{sep}-97$ & 248 & 0200 & 480 & 100.0 & 48.00 & 2 & 9.175 & 1817 & 8.260 & 0.87 & 1.30 & 4.18 & 20.8 & 9 \\
\hline $5-\operatorname{sep}-97$ & 248 & 1000 & 480 & 100.0 & 48.00 & 3 & 0.000 & 3147 & 3.969 & 0.96 & 1.30 & 4.18 & 23.0 & 9 \\
\hline $5-\operatorname{sep}-97$ & 248 & 1800 & 480 & 100.0 & 48.00 & 4 & 0.000 & 5361 & 0.080 & 0.65 & 1.30 & 4.18 & 15.6 & \\
\hline $6-\operatorname{sep}-97$ & 249 & 0200 & 480 & 100.0 & 48.00 & 1 & 0.000 & 1138 & 0.087 & 0.78 & 1.60 & 4.18 & 18.7 & \\
\hline
\end{tabular}


TABLE 2 (Cont'd)

RESULTS OF ARSA SAMPLING AND ANALYSIS

SITE NUMBER: $805 \mathrm{X}$

\begin{tabular}{|c|c|c|c|c|c|c|c|c|c|c|c|c|c|c|}
\hline $\begin{array}{c}\text { Sample } \\
\text { Date }\end{array}$ & $\begin{array}{c}\text { J } \\
\text { Date }\end{array}$ & $\begin{array}{l}\text { Clock } \\
\text { time }\end{array}$ & $\begin{array}{l}\text { Sample } \\
\text { Dur. } \\
\text { (Min) }\end{array}$ & $\begin{array}{l}\text { Flow } \\
\text { Rate } \\
\text { (L/Min) }\end{array}$ & $\begin{array}{l}\text { Samp. } \\
\text { Vol. } \\
\text { (SCM) }\end{array}$ & $\begin{array}{l}\text { Det. } \\
\text { Celi } \\
\text { No. }\end{array}$ & $\begin{array}{c}\mathrm{Xe}-133 \\
(\mathrm{~B}-\mathrm{G}) \\
(\mathrm{pCi} / \mathrm{SCM})\end{array}$ & $\begin{array}{l}\text { Coll. } \\
\text { Bot. } \\
\text { No. }\end{array}$ & $\begin{array}{r}\mathrm{Xe}-133 \\
\text { (Anal) } \\
\text { (pCi/SCM) }\end{array}$ & $\begin{array}{c}\text { Xe } \\
\text { Recov. } \\
\text { (Anal) } \\
\text { (mL) }\end{array}$ & $\begin{array}{c}\text { Xe } \\
\text { Recov. } \\
\text { (RGA) } \\
\text { (mL) }\end{array}$ & $\begin{array}{c}\text { Exp } \\
\text { Total } \\
\text { Xe } \\
(m L)\end{array}$ & $\begin{array}{c}\text { Xe } \\
\text { Recov } \\
(\text { Anal. }) \\
\left(\frac{\circ}{\delta}\right)\end{array}$ & $\begin{array}{l}\text { Flag } \\
\text { No. }\end{array}$ \\
\hline $6-\operatorname{sep}-97$ & 249 & 1000 & 480 & 100.0 & 48.00 & 2 & 0.000 & F598 & 0.080 & 1.02 & 1.60 & 4.18 & 24.4 & \\
\hline $6-\mathrm{Sep}-97$ & 249 & 1800 & 480 & 100.0 & 48.00 & 3 & 0.000 & 3161 & 0.101 & 0.91 & 1.50 & 4.18 & 21.8 & \\
\hline $7-$ Sep- 97 & 250 & 0200 & 480 & 100.0 & 48.00 & 4 & 0.161 & 4091 & 0.099 & 0.97 & 1.50 & 4.18 & 23.2 & \\
\hline $7-$ Sep- 97 & 250 & 1000 & 480 & 100.0 & 48.00 & 1 & 0.056 & 0153 & 0.074 & 0.99 & 1.70 & 4.18 & 23.7 & \\
\hline 7-Sep-97 & 250 & 1800 & 480 & 100.0 & 48.00 & 2 & 0.043 & 3822 & 0.000 & 0.00 & 1.60 & 4.18 & & 4 \\
\hline $8-$ Sep- 97 & 251 & 0200 & 480 & 100.0 & 48.00 & 3 & 0.123 & 2947 & 0.102 & 0.81 & 1.60 & 4.18 & 19.4 & \\
\hline $8-$ Sep- 97 & 251 & 1000 & 480 & 100.0 & 48.00 & 4 & 0.275 & 0382 & 0.210 & 0.91 & 1.40 & 4.18 & 21.8 & \\
\hline $8-$ Sep- 97 & 251 & 1800 & 480 & 100.0 & 48.00 & 1 & 0.144 & 1698 & 0.098 & 1.06 & 1.40 & 4.18 & 25.4 & \\
\hline $9-$ Sep- 97 & 252 & 0200 & 480 & 100.0 & 48.00 & 2 & 0.098 & 0842 & 0.080 & 0.74 & 1.80 & 4.18 & 17.7 & \\
\hline $9-$ Sep-97 & 252 & 1000 & 480 & 100.0 & 48.00 & 3 & 0.242 & 3505 & 0.014 & 0.91 & 1.80 & 4.18 & 21.8 & 10 \\
\hline $9-$ Sep- 97 & 252 & 1800 & 480 & 100.0 & 48.00 & 4 & 0.313 & 0579 & 0.203 & 0.80 & 1.30 & 4.18 & 19.2 & \\
\hline 10-Sep-97 & 253 & 0200 & 480 & 100.0 & 48.00 & 1 & 0.312 & 0797 & 0.000 & 0.00 & 0.00 & 4.18 & & 12 \\
\hline $10-$ Sep- 97 & 253 & 1000 & 480 & 100.0 & 48.00 & 2 & 0.000 & 2075 & 0.047 & 0.71 & 1.90 & 4.18 & 17.0 & \\
\hline 11 -Sep-97 & 254 & 0200 & 480 & 100.0 & 48.00 & 3 & 0.034 & E667 & 0.038 & 0.33 & 1.00 & 4.18 & 7.9 & \\
\hline $11-$ Sep- 97 & 254 & 1000 & 480 & 100.0 & 48.00 & 4 & 0.026 & 5174 & 0.038 & 0.27 & 0.90 & 4.18 & 6.5 & \\
\hline $24-$ Oct -97 & 297 & 1000 & 480 & 100.0 & 48.00 & 1 & 0.175 & 0797 & 0.215 & 0.74 & 1.20 & 4.18 & 17.7 & 10 \\
\hline $24-0 c t-97$ & 297 & 1800 & 480 & 100.0 & 48.00 & 2 & 0.174 & 0955 & 0.073 & 0.67 & I. 10 & 4.18 & 16.0 & \\
\hline $25-0 c t-97$ & 298 & 0200 & 480 & 100.0 & 48.00 & 3 & 0.900 & 1176 & 0.024 & 0.94 & 1.40 & 4.18 & 22.5 & \\
\hline
\end{tabular}


TABLE 2 (Cont'd)

RESULTS OF ARSA SAMPLING AND ANALYSIS

SITE NUMBER: $805 \mathrm{X}$

\begin{tabular}{|c|c|c|c|c|c|c|c|c|c|c|c|c|c|c|}
\hline $\begin{array}{c}\text { Sample } \\
\text { Date }\end{array}$ & $\begin{array}{c}\text { J } \\
\text { Date }\end{array}$ & $\begin{array}{l}\text { Clock } \\
\text { time }\end{array}$ & $\begin{array}{l}\text { Sample } \\
\text { Dur. } \\
\text { (Min) }\end{array}$ & $\begin{array}{l}\text { Flow } \\
\text { Rate } \\
\text { (L/Min) }\end{array}$ & $\begin{array}{l}\text { Samp. } \\
\text { Vol. } \\
\text { (SCM) }\end{array}$ & $\begin{array}{l}\text { Det. } \\
\text { Cell } \\
\text { No. }\end{array}$ & $\begin{array}{c}\mathrm{Xe}-133 \\
(\mathrm{~B}-\mathrm{G}) \\
(\mathrm{pCi} / \mathrm{sCM})\end{array}$ & $\begin{array}{l}\text { Coll. } \\
\text { Bot. } \\
\text { No. }\end{array}$ & $\begin{array}{r}\mathrm{Xe}-133 \\
\text { (Anal) } \\
\text { (pCi/sCM) }\end{array}$ & $\begin{array}{c}\text { Xe } \\
\text { Recov. } \\
\text { (Anal) } \\
\text { (mL) }\end{array}$ & $\begin{array}{c}\text { Xe } \\
\text { Recov. } \\
\text { (RGA) } \\
\text { (mL) }\end{array}$ & $\begin{array}{c}\text { Exp } \\
\text { Total } \\
\text { Xe } \\
(\mathrm{mL})\end{array}$ & $\begin{array}{c}\text { Xe } \\
\text { Recov } \\
(\text { Anal. }) \\
\left(\frac{\circ}{0}\right)\end{array}$ & $\begin{array}{c}\text { Flag } \\
\text { No. }\end{array}$ \\
\hline- Oct -97 & 298 & 1000 & 480 & 100.0 & 48.00 & 4 & 0.026 & $\mathrm{E} 667$ & 0.024 & 0.60 & 0.90 & 4.18 & 14.4 & \\
\hline $5-$ Oct -97 & 298 & 1800 & 480 & 100.0 & 48.00 & 1 & 0.313 & 5473 & 0.352 & 0.86 & 1.50 & 4.18 & 20.6 & \\
\hline $6-0 c t-97$ & 299 & 0200 & 480 & 100.0 & 48.00 & 2 & 1.330 & 2278 & 1.382 & 0.65 & 1.20 & 4.18 & 15.6 & \\
\hline $6-$ oct -97 & 299 & 1000 & 480 & 100.0 & 48.00 & 3 & 0.558 & 2632 & 0.702 & 0.74 & 1.20 & 4.18 & 17.7 & \\
\hline $6-0 c t-97$ & 299 & 1800 & 480 & 100.0 & 48.00 & 4 & 0.307 & 2186 & 0.272 & 0.70 & 1.10 & 4.18 & 16.8 & \\
\hline $7-0 c t-97$ & 300 & 0200 & 480 & 100.0 & 48.00 & 1 & 0.096 & 3505 & 0.133 & 0.77 & 1.30 & 4.18 & 18.4 & \\
\hline $8-$ oct -97 & 301 & 1000 & 480 & 100.0 & 48.00 & 1 & 0.030 & 0660 & 0.033 & 0.80 & 1.40 & 4.18 & 19.2 & \\
\hline $8-0 c t-97$ & 301 & 1800 & 480 & 100.0 & 48.00 & 2 & 0.052 & 0382 & 0.061 & 0.50 & 1.30 & 4.18 & 12.0 & \\
\hline $9-$ Oct -97 & 302 & 0200 & 480 & 100.0 & 48.00 & 3 & 0.030 & 6101 & 0.068 & 0.70 & 1.20 & 4.18 & 16.8 & \\
\hline $9-0 c t-97$ & 302 & 1000 & 480 & 100.0 & 48.00 & 4 & 0.113 & 2947 & 0.091 & 0.70 & 1.00 & 4.18 & 16.8 & 10 \\
\hline $9-$ Oct -97 & 302 & 1800 & 480 & 100.0 & 48.00 & 1 & 0.166 & 5174 & 0.072 & 0.80 & 1.30 & 4.18 & 19.2 & 10 \\
\hline $0-0 c t-97$ & 303 & 0200 & 480 & 100.0 & 48.00 & 2 & 0.163 & 1081 & 0.061 & 0.50 & 1.30 & 4.18 & 12.0 & 10 \\
\hline 0 -Nov- 97 & 334 & 1000 & 480 & 100.0 & 48.00 & 4 & 0.358 & 1465 & 0.261 & 0.50 & 0.70 & 4.18 & 12.0 & \\
\hline 0 -Nov-97 & 334 & 1800 & 480 & 100.0 & 48.00 & 1 & 0.753 & 4073 & 0.980 & 0.50 & 0.90 & 4.18 & 12.0 & \\
\hline $1-\operatorname{Dec}-97$ & 335 & 0200 & 480 & 100.0 & 48.00 & 2 & 9.909 & 3147 & 9.240 & 0.50 & 0.90 & 4.18 & 12.0 & \\
\hline $1-\operatorname{Dec}-97$ & 335 & 1000 & 480 & 100.0 & 48.00 & 3 & 107.100 & 0348 & 97.650 & 0.60 & 0.80 & 4.18 & 14.4 & 11 \\
\hline 1-Dec-97 & 335 & 1800 & 480 & 100.0 & 48.00 & 4 & 95.800 & 0153 & 86.700 & 0.50 & 0.80 & 4.18 & 12.0 & 11 \\
\hline
\end{tabular}




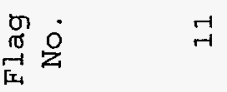

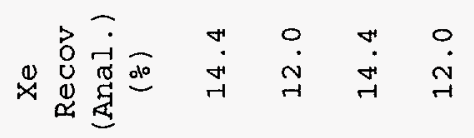

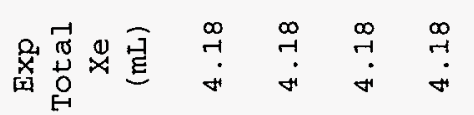

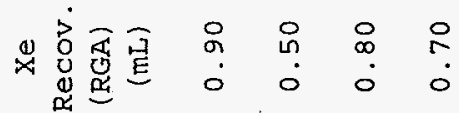

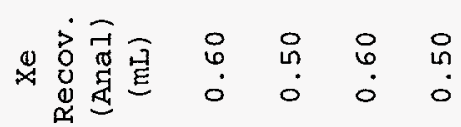

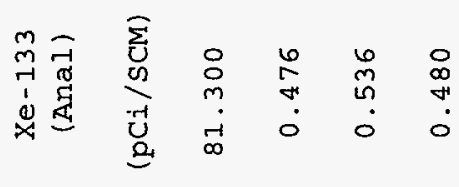

竞荅卒

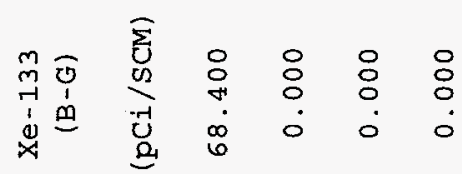

过藏芸

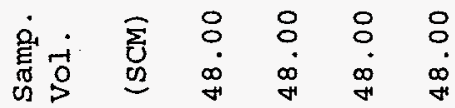

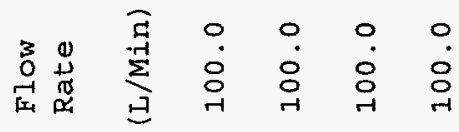

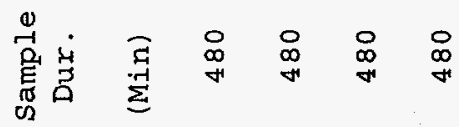

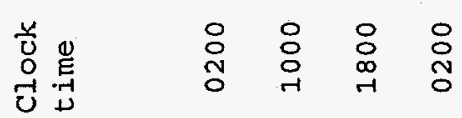

D

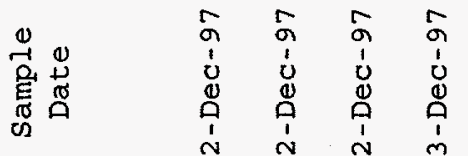


TABLE 2 (Cont'd)

RESULTS OF ARSA SAMPLING AND ANALYSIS

IDENTIFICATION OF FLAG CODES

Code No.

Description

Xe-133 concentration obtained from real-time beta-gamma radioassay (No archive bottle collected)

Sample contained $\mathrm{Xe}-135$, archive bottle analyzed by AFTAC

Sample contaminated with Radon

Sample lost in analysis

Sample air stream at $20^{\circ} \mathrm{C}$ and $20 \%$ Humidity

Sample air stream at $20^{\circ} \mathrm{C}$ and $50 \%$ Humidity

Sample air stream at $20^{\circ} \mathrm{C}$ and $90 \%$ Humidity

Possible leak in ARSA archive bottle \#6

Contains 20 pCi Xe^133 (Env. Chamber Experiment)

Radon Environmental Chamber Test @ 3 pCi/Liter Rn-222

11

Contains $-100 \mathrm{pCi} \mathrm{Xe}^{\wedge} 133^{`}$ (Env. Chamber Experiment

Low xenon gas recovery 
TABLE 3

RESULTS OF ARSA DETECTOR BACKGROUND DETERMINATIONS

SITE NUMBER: $805 \mathrm{X}$

\begin{tabular}{|c|c|c|c|c|c|c|c|c|c|c|c|c|c|c|}
\hline $\begin{array}{l}\text { Sample } \\
\text { Date }\end{array}$ & $\begin{array}{c}\text { J } \\
\text { Date }\end{array}$ & $\begin{array}{l}\text { Clock } \\
\text { time } \\
\text { (Loc) }\end{array}$ & $\begin{array}{l}\text { Count } \\
\text { Time } \\
\text { (Min) }\end{array}$ & $\begin{array}{l}\text { Det. } \\
\text { Celi } \\
\text { No. }\end{array}$ & $\begin{array}{l}\text { Total } \\
\text { Counts } \\
\text { NaI (1) } \\
(\mathrm{b}-\mathrm{g})\end{array}$ & $\begin{array}{l}\text { Total } \\
\text { Counts } \\
\text { NaI (2) } \\
(b-g)\end{array}$ & $\begin{array}{l}\text { Total } \\
\text { Counts } \\
31 \mathrm{keV} \\
(b-g)\end{array}$ & $\begin{array}{l}\text { Total } \\
\text { Counts } \\
81 \mathrm{keV} \\
(\mathrm{b}-\mathrm{g})\end{array}$ & $\begin{array}{l}\text { Total } \\
\text { Counts } \\
\text { Radon* } \\
(b-g)\end{array}$ & $\begin{array}{c}\text { CPM } \\
\operatorname{NaI}(1,2) \\
\text { Gross } \\
(b-g)\end{array}$ & $\begin{array}{c}\mathrm{CPM} \\
\mathrm{NaI}(1,2) \\
31 \mathrm{keV} \\
(\mathrm{b}-\mathrm{g})\end{array}$ & $\begin{array}{c}\mathrm{CPM} \\
\mathrm{NaI}(1,2) \\
81 \mathrm{keV} \\
(\mathrm{b}-\mathrm{g})\end{array}$ & $\begin{array}{l}\text { CPM } \\
\text { Total } \\
\text { Rn } \\
(b-g)\end{array}$ & $\begin{array}{c}\text { Flag } \\
\text { No. }\end{array}$ \\
\hline 10-Apr-97 & 100 & 0739 & 960 & 1 & 398.0 & 356.0 & 91.0 & 109.0 & & .79 & .09 & .11 & & 5 \\
\hline $10-A p r-97$ & 100 & 0739 & 960 & 2 & 313.0 & 321.0 & 56.0 & 44.0 & & .66 & .06 & .05 & & 5 \\
\hline 10-Apr-97 & 100 & 0739 & 960 & 3 & 367.0 & 401.0 & 69.0 & 85.0 & & .80 & .07 & .09 & & 5 \\
\hline 10-Apr-97 & 100 & 0739 & 960 & 4 & 543.0 & 598.0 & 506.0 & 177.0 & & 1.19 & .53 & .18 & & 5 \\
\hline 18-Apr-97 & 108 & 1135 & 960 & 1 & 225.0 & 252.0 & 36.0 & 41.0 & & .50 & .04 & .04 & & 5 \\
\hline $18-\operatorname{Apr}-97$ & 108 & 1135 & 960 & 2 & 262.0 & 241.0 & 27.0 & 30.0 & & .52 & .03 & .03 & & 5 \\
\hline 18-Apr-97 & 108 & 1135 & 960 & 3 & 325.0 & 300.0 & 24.0 & 59.0 & & .65 & .03 & .06 & & 5 \\
\hline 18-Apr-97 & 108 & 1135 & 960 & 4 & 402.0 & 367.0 & 224.0 & 70.0 & & .80 & .23 & .07 & & 5 \\
\hline $21-A p r-97$ & 111 & 1128 & 934 & 2 & 744.0 & 708.0 & & & & 1.55 & & & & 4 \\
\hline 21-Apr-97 & 111 & 1925 & 933 & 3 & 373.0 & 433.0 & & & & .86 & & & & 1 \\
\hline $22-\mathrm{Apr}-97$ & 112 & 0321 & 934 & 4 & 616.0 & 607.0 & & & & 1.31 & & & & 2 \\
\hline 22-Apr-97 & 112 & 1118 & 934 & 1 & 420.0 & 417.0 & & & & .90 & & & & 3 \\
\hline 22-Apr-97 & 112 & 1915 & 933 & 2 & 521.0 & 516.0 & & & & 1.11 & & & & 4 \\
\hline 23-Apr-97 & 113 & 0311 & 933 & 3 & $446.0 \times$ & 429.0 & & & & .94 & & & & 1 \\
\hline 23-Apr-97 & 113 & 1107 & 933 & 4 & 509.0 & 476.0 & & & & 1.06 & & & & 2 \\
\hline $23-\mathrm{Apr}-97$ & 113 & 1904 & 933 & 1 & 385.0 & 367.0 & & & & .81 & & & & 3 \\
\hline 24-Apr-97 & 114 & 0300 & 937 & 2 & 505.0 & 454.0 & & & & 1.02 & & & & 4 \\
\hline 24 - Apr-97 & 114 & 1056 & 940 & 3 & 364.0 & 386.0 & & & & .80 & & & & 1 \\
\hline
\end{tabular}


TABLE 3 (Cont'd)

RESULTS OF ARSA DETECTOR BACKGROUND DETERMINATIONS

SITE NUMBER: $805 \mathrm{X}$

\begin{tabular}{|c|c|c|c|c|c|c|c|c|c|c|c|c|c|c|}
\hline $\begin{array}{l}\text { Sample } \\
\text { Date }\end{array}$ & $\begin{array}{c}\text { J } \\
\text { Date }\end{array}$ & $\begin{array}{l}\text { Clock } \\
\text { time } \\
\text { (Loc) }\end{array}$ & $\begin{array}{l}\text { Count } \\
\text { Time } \\
\text { (Min) }\end{array}$ & $\begin{array}{l}\text { Det. } \\
\text { Celi } \\
\text { No. }\end{array}$ & $\begin{array}{l}\text { Total } \\
\text { Counts } \\
\text { NaI (1) } \\
(b-g)\end{array}$ & $\begin{array}{l}\text { Total } \\
\text { Counts } \\
\operatorname{NaI}(2) \\
(b-g)\end{array}$ & $\begin{array}{l}\text { Total } \\
\text { Counts } \\
31 \mathrm{keV} \\
(\mathrm{b}-\mathrm{g})\end{array}$ & $\begin{array}{l}\text { Total } \\
\text { Counts } \\
81 \mathrm{keV} \\
(\mathrm{b}-\mathrm{g})\end{array}$ & $\begin{array}{l}\text { Total } \\
\text { Counts } \\
\text { Radon* } \\
(\mathrm{b}-\mathrm{g})\end{array}$ & $\begin{array}{c}\mathrm{CPM} \\
\operatorname{NaI}(1,2) \\
\text { Gross } \\
(\mathrm{b}-\mathrm{g})\end{array}$ & $\begin{array}{c}\mathrm{CPM} \\
\mathrm{NaI}(1,2) \\
31 \mathrm{keV} \\
(\mathrm{b}-\mathrm{g})\end{array}$ & $\begin{array}{c}\mathrm{CPM} \\
\mathrm{NaI}(1,2) \\
81 \mathrm{keV} \\
(\mathrm{b}-\mathrm{g})\end{array}$ & $\begin{array}{l}\text { CPM } \\
\text { Total } \\
\text { Rn } \\
(b-g)\end{array}$ & $\begin{array}{c}\text { Flag } \\
\text { No. }\end{array}$ \\
\hline 30-Apr-97 & 120 & 0300 & 941 & 4 & 10321.0 & 10387.0 & & & & 22.01 & & & & 2,6 \\
\hline $30-A p r-97$ & 120 & 1100 & 941 & 1 & 488.0 & 488.0 & & & & 1.04 & & & & 3 \\
\hline 30-Apr-97 & 120 & 1900 & 940 & 2 & 13350.0 & 13303.0 & & & & 28.35 & & & & 4,6 \\
\hline 1-May-97 & 121 & 0300 & 939 & 3 & 1831.0 & 1770.0 & & & & 3.83 & & & & 1 \\
\hline 1-May-97 & 121 & 1100 & 939 & 4 & 2889.0 & 2771.0 & & & & 6.03 & & & & 2,6 \\
\hline 5-Aug- 97 & 217 & 0200 & 940 & 1 & 585.0 & 563.0 & 63.0 & 234.0 & 266.0 & 1.22 & .07 & .25 & .28 & \\
\hline 5-Aug- 97 & 217 & 1000 & 940 & 2 & 385.0 & 335.0 & 52.0 & 85.0 & 58.0 & .77 & .06 & .09 & .06 & \\
\hline 5-Aug-97 & 217 & 1800 & 940 & 3 & 628.0 & 650.0 & 47.0 & 211.0 & 310.0 & 1.36 & .05 & .22 & .33 & \\
\hline 6-Aug- 97 & 218 & 0200 & 940 & 4 & 469.0 & 568.0 & 37.0 & 140.0 & 228.0 & 1.10 & .04 & .15 & .24 & \\
\hline 6-Aug- 97 & 218 & 1000 & 940 & 1 & 396.0 & 404.0 & 56.0 & 144.0 & & .85 & .06 & .15 & & \\
\hline 6-Aug- 97 & 218 & 1800 & 949 & 2 & 908.0 & 891.0 & 181.0 & 287.0 & & 1.90 & .19 & .30 & & \\
\hline 7-Aug-97 & 219 & 0200 & 940 & 3 & 626.0 & 628.0 & 121.0 & 266.0 & & 1.33 & .13 & 28 & & \\
\hline 7-Aug - 97 & 219 & 1000 & 940 & 4 & 1148.0 & 1149.0 & 177.0 & 589.0 & 609.0 & 2.44 & .19 & .63 & .65 & \\
\hline 24-Aug-97 & 236 & 1400 & 941 & 3 & 1454.0 & 1310.0 & 354.0 & 712.0 & 1086.0 & 2.94 & .38 & .76 & 1.15 & \\
\hline 24-Aug-97 & 236 & 2300 & 941 & 4 & 1393.0 & 1350.0 & 260.0 & 707.0 & 1149.0 & 2.91 & .28 & .75 & 1.22 & \\
\hline 25-Aug-97 & 237 & 0600 & 941 & 1 & 982.0 & 923.0 & 161.0 & 507.0 & 752.0 & 2.02 & .17 & .54 & .80 & \\
\hline 25-Aug-97 & 237 & 1400 & 941 & 2 & 1506.0 & 1469.0 & 286.0 & 755.0 & 1172.0 & 3.16 & .30 & .80 & 1.25 & \\
\hline 25-Aug-97 & 237 & 2300 & 941 & 3 & 1131.0 & 1073.0 & 210.0 & 580.0 & 961.0 & 2.34 & .22 & .62 & 1.02 & \\
\hline 26-Aug - 97 & 238 & 0600 & 941 & 4 & 1340.0 & 1330.0 & 186.0 & 656.0 & 1106.0 & 2.84 & .20 & .70 & 1.18 & \\
\hline
\end{tabular}


TABLE 3 (Cont' $d$ )

RESULTS OF ARSA DETECTOR BACKGROUND DETERMINATIONS

SITE NUMBER: $805 \mathrm{X}$

\begin{tabular}{|c|c|c|c|c|c|c|c|c|c|c|c|c|c|}
\hline $\begin{array}{l}\text { Sample } \\
\text { Date }\end{array}$ & $\begin{array}{c}\mathrm{J} \\
\text { Date }\end{array}$ & $\begin{array}{l}\text { Clock } \\
\text { time } \\
\text { (Loc) }\end{array}$ & $\begin{array}{l}\text { Count } \\
\text { Time } \\
\text { (Min) }\end{array}$ & $\begin{array}{l}\text { Det. } \\
\text { Cell } \\
\text { No. }\end{array}$ & $\begin{array}{l}\text { Total } \\
\text { Counts } \\
\text { NaI (1) } \\
(b-g)\end{array}$ & $\begin{array}{l}\text { Total } \\
\text { Counts } \\
\text { NaI (2) } \\
(b-g)\end{array}$ & $\begin{array}{l}\text { Total } \\
\text { Counts } \\
31 \mathrm{keV} \\
(\mathrm{b}-\mathrm{g})\end{array}$ & $\begin{array}{l}\text { Total } \\
\text { Counts } \\
81 \mathrm{keV} \\
(\mathrm{b}-\mathrm{g})\end{array}$ & $\begin{array}{l}\text { Total } \\
\text { Counts } \\
\text { Radon* } \\
(b-g)\end{array}$ & $\begin{array}{c}\text { CPM } \\
\operatorname{NaI}(1,2) \\
\text { Gross } \\
(b-g)\end{array}$ & $\begin{array}{c}\text { CPM } \\
\mathrm{NaI}(1,2) \\
31 \mathrm{keV} \\
(\mathrm{b}-\mathrm{g})\end{array}$ & $\begin{array}{c}\mathrm{CPM} \\
\mathrm{NaI}(1,2) \\
81 \mathrm{keV} \\
(\mathrm{b}-\mathrm{g})\end{array}$ & $\begin{array}{l}\text { CPM } \\
\text { Total } \\
\text { Rn } \\
(\mathrm{b}-\mathrm{g})\end{array}$ \\
\hline 26-Aug- 97 & 238 & 1400 & 941 & 1 & 1050.0 & 989.0 & 183.0 & 518.0 & 862.0 & 2.17 & .19 & .55 & .92 \\
\hline 26-Aug-97 & 238 & 2300 & 941 & 2 & 1307.0 & 1298.0 & 270.0 & 640.0 & 1102.0 & 2.77 & .29 & .68 & 1.17 \\
\hline 27-Aug- 97 & 239 & 0600 & 941 & 3 & 1079.0 & 962.0 & 183.0 & 497.0 & 835.0 & 2.17 & .19 & .53 & .89 \\
\hline 27-Aug-97 & 239 & 1400 & 941 & 4 & 1170.0 & 1193.0 & 217.0 & 610.0 & 973.0 & 2.51 & .23 & .65 & 1.03 \\
\hline 27-Aug- 97 & 239 & 2300 & 941 & 1 & 991.0 & 965.0 & 274.0 & 523.0 & 692.0 & 2.08 & .29 & .56 & .74 \\
\hline 28-Aug-97 & 240 & 0600 & 941 & 2 & 1384.0 & 1291.0 & 278.0 & 694.0 & 1093.0 & 2.84 & .30 & .74 & 1.16 \\
\hline 29-Aug-97 & 241 & 1400 & 944 & 3 & 724.0 & 672.0 & 161.0 & 283.0 & 542.0 & 1.48 & .17 & .30 & .57 \\
\hline 29-Aug-97 & 241 & 2300 & 944 & 4 & 757.0 & 727.0 & 116.0 & 347.0 & 612.0 & 1.57 & .12 & .37 & .65 \\
\hline 30-Aug- 97 & 242 & 0600 & 944 & 1 & 827.0 & 756.0 & 140.0 & 418.0 & 592.0 & 1.68 & .15 & .44 & .63 \\
\hline $30-$ Aug- 97 & 242 & 1400 & 944 & 2 & 1377.0 & 1370.0 & 225.0 & 715.0 & 1151.0 & $2.9 I$ & .24 & .76 & 1.22 \\
\hline 30-Aug- 97 & 242 & 2300 & 944 & 3 & 1205.0 & 1062.0 & 234.0 & 539.0 & 918.0 & 2.40 & .25 & .57 & .97 \\
\hline 31-Aug - 97 & 243 & 0600 & 944 & 4 & 2062.0 & 1969.0 & 912.0 & 1301.0 & 1130.0 & 4.27 & .97 & 1.38 & 1.20 \\
\hline 31-Aug- 97 & 243 & 1400 & 944 & 1 & 1408.0 & 1485.0 & 501.0 & 883.0 & 874.0 & 3.06 & .53 & .94 & .93 \\
\hline 31-Aug - 97 & 243 & 2300 & 944 & 2 & $1808.0 \%$ & 1808.0 & 533.0 & 937.0 & 1347.0 & 3.83 & .56 & .99 & 1.43 \\
\hline $1-$ Sep- 97 & 244 & 0600 & 944 & 3 & 1599.0 & 1535.0 & 527.0 & 905.0 & 1029.0 & 3.32 & .56 & .96 & 1.09 \\
\hline 1-Sep- 97 & 244 & 1400 & 944 & 4 & 2140.0 & 2118.0 & 657.0 & 1230.0 & 1545.0 & 4.51 & .70 & 1.30 & 1.64 \\
\hline $1-$ Sep- 97 & 244 & 2300 & 944 & 1 & 1595.0 & 1575.0 & 559.0 & 925.0 & 488.0 & 3.36 & .59 & .98 & .52 \\
\hline $1-$ Sep- 97 & 244 & 2300 & 944 & 1 & 1595.0 & 1575.0 & 559.0 & 925.0 & 1015.0 & 3.36 & .59 & .98 & 1.08 \\
\hline $2-\operatorname{sep}-97$ & 245 & 0600 & 944 & 2 & 2018.0 & 1946.0 & 545.0 & 1089.0 & 1479.0 & 4.20 & .58 & 1.15 & 1.57 \\
\hline
\end{tabular}


TABLE 3 (Cont'd)

RESULTS OF ARSA DETECTOR BACKGROUND DETERMINATIONS

SITE NUMBER: $805 \mathrm{X}$

\begin{tabular}{|c|c|c|c|c|c|c|c|c|c|c|c|c|c|}
\hline $\begin{array}{l}\text { Sample } \\
\text { Date }\end{array}$ & $\begin{array}{c}\mathrm{J} \\
\text { Date }\end{array}$ & $\begin{array}{l}\text { Clock } \\
\text { time } \\
\text { (Loc) }\end{array}$ & $\begin{array}{l}\text { Count } \\
\text { Time } \\
\text { (Min) }\end{array}$ & $\begin{array}{l}\text { Det. } \\
\text { Cell } \\
\text { No. }\end{array}$ & $\begin{array}{l}\text { Total } \\
\text { Counts } \\
\text { NaI (1) } \\
(b-g)\end{array}$ & $\begin{array}{l}\text { Total } \\
\text { Counts } \\
\text { NaI (2) } \\
(b-g)\end{array}$ & $\begin{array}{l}\text { Total } \\
\text { Counts } \\
31 \mathrm{keV} \\
(\mathrm{b}-\mathrm{g})\end{array}$ & $\begin{array}{l}\text { Total } \\
\text { Counts } \\
81 \text { keV } \\
(b-g)\end{array}$ & $\begin{array}{l}\text { Total } \\
\text { Counts } \\
\text { Radon* } \\
(b-g)\end{array}$ & $\begin{array}{c}\text { CPM } \\
\mathrm{NaI}(1,2) \\
\text { Gross } \\
(b-g)\end{array}$ & $\begin{array}{c}\text { CPM } \\
\operatorname{NaI}(1,2) \\
31 \mathrm{keV} \\
(b-g)\end{array}$ & $\begin{array}{c}\text { CPM } \\
\mathrm{NaI}(1,2) \\
81 \mathrm{keV} \\
(\mathrm{b}-\mathrm{g})\end{array}$ & $\begin{array}{l}\text { CPM } \\
\text { Total } \\
\text { Rn } \\
(b-g)\end{array}$ \\
\hline $2-\operatorname{sep}-97$ & 245 & 1400 & 944 & 3 & 1635.0 & 1547.0 & 421.0 & 1093.0 & 1208.0 & 3.37 & .45 & 1.16 & 1.28 \\
\hline $2-\operatorname{sep}-97$ & 245 & 2300 & 944 & 4 & 1972.0 & 1932.0 & 531.0 & 1062.0 & 1474.0 & 4.14 & .56 & 1.13 & 1.56 \\
\hline 3-sep-97 & 246 & 0600 & 944 & 1 & 1315.0 & 1241.0 & 310.0 & 730.0 & 919.0 & 2.71 & .33 & .77 & .97 \\
\hline 3-Sep- 97 & 246 & 1400 & 944 & 2 & 2552.0 & 2517.0 & 439.0 & 1417.0 & 2138.0 & 5.37 & .47 & 1.50 & 2.26 \\
\hline 3-Sep-97 & 246 & 2300 & 944 & 3 & 1842.0 & 1757.0 & 339.0 & 1030.0 & 1443.0 & 3.81 & .36 & 1.09 & 1.53 \\
\hline $4-\operatorname{sep}-97$ & 247 & 0600 & 944 & 4 & 1980.0 & 1961.0 & 867.0 & 1191.0 & 1186.0 & 4.17 & .92 & 1.26 & 1.26 \\
\hline $4-\operatorname{sep}-97$ & 247 & 1400 & 944 & 1 & 1640.0 & 1597.0 & 939.0 & 1049.0 & 738.0 & 3.43 & .99 & 1.11 & .78 \\
\hline $4-\operatorname{sep}-97$ & 247 & 2300 & 944 & 2 & 2980.0 & 2982.0 & 1956.0 & 1999.0 & 1276.0 & 6.32 & 2.07 & 2.12 & 1.35 \\
\hline $5-\operatorname{sep}-97$ & 248 & 0600 & 944 & 3 & 2520.0 & 2326.0 & 1718.0 & 1683.0 & 836.0 & 5.13 & 1.82 & 1.78 & .89 \\
\hline $5-\operatorname{sep}-97$ & 248 & 1400 & 944 & 4 & 2993.0 & 2855.0 & 2212.0 & 2017.0 & 958.0 & 6.19 & 2.34 & 2.14 & 1.01 \\
\hline 5-Sep-97 & 248 & 2300 & 944 & 1 & 2490.0 & 2509.0 & 2095.0 & 1806.0 & 640.0 & 5.30 & 2.22 & 1.91 & .68 \\
\hline $6-$ Sep-97 & 249 & 0600 & 944 & 2 & 3417.0 & 3406.0 & 2671.0 & 2347.0 & 1123.0 & 7.23 & 2.83 & 2.49 & 1.19 \\
\hline $6-\operatorname{sep}-97$ & 249 & 1400 & 944 & 3 & 2381.0 & 2234.0 & 1530.0 & 1563.0 & 889.0 & 4.89 & 1.62 & 1.66 & .94 \\
\hline $6-\operatorname{sep}-97$ & 249 & 2300 & 944 & 4 & 1950.0. & 1891.0 & 825.0 & 1195.0 & 1080.0 & 4.07 & .87 & 1.27 & 1.14 \\
\hline $7-$ Sep-97 & 250 & 0600 & 944 & 1 & 2183.0 & 2055.0 & 853.0 & 1285.0 & 1348.0 & 4.49 & .90 & 1.36 & 1.43 \\
\hline 7-Sep-97 & 250 & 1400 & 944 & 2 & 3926.0 & 3665.0 & 1245.0 & 2328.0 & 2631.0 & 8.04 & 1.32 & 2.47 & 2.79 \\
\hline $7-$ Sep-97 & 250 & 2300 & 944 & 3 & 2207.0 & 2069.0 & 877.0 & 1316.0 & 1305.0 & 4.53 & .93 & 1.39 & 1.38 \\
\hline $8-$ Sep-97 & 251 & 0600 & 944 & 4 & 2625.0 & 2678.0 & 830.0 & 1586.0 & 1910.0 & 5.62 & .88 & 1.68 & 2.02 \\
\hline 8-Sep-97 & 251 & 1400 & 944 & 1 & 2222.0 & 2184.0 & 727.0 & 1360.0 & 1467.0 & 4.67 & .77 & 1.44 & 1.55 \\
\hline
\end{tabular}


TABLE 3 (Cont'd)

RESULTS OF ARSA DETECTOR BACKGROUND DETERMINATIONS

SITE NUMBER: $805 \mathrm{X}$

\begin{tabular}{|c|c|c|c|c|c|c|c|c|c|c|c|c|c|c|}
\hline $\begin{array}{l}\text { Sample } \\
\text { Date }\end{array}$ & $\begin{array}{c}\mathcal{J} \\
\text { Date }\end{array}$ & $\begin{array}{l}\text { Clock } \\
\text { time } \\
\text { (Loc) }\end{array}$ & $\begin{array}{l}\text { Count } \\
\text { Time } \\
\text { (Min) }\end{array}$ & $\begin{array}{l}\text { Det. } \\
\text { Cell } \\
\text { No. }\end{array}$ & $\begin{array}{l}\text { Total } \\
\text { Counts } \\
\text { NaI (1) } \\
(b-g)\end{array}$ & $\begin{array}{l}\text { Total } \\
\text { Counts } \\
\text { NaI (2) } \\
(b-g)\end{array}$ & $\begin{array}{l}\text { Total } \\
\text { Counts } \\
31 \mathrm{keV} \\
(\mathrm{b}-\mathrm{g})\end{array}$ & $\begin{array}{l}\text { Total } \\
\text { Counts } \\
81 \mathrm{keV} \\
(\mathrm{b}-\mathrm{g})\end{array}$ & $\begin{array}{l}\text { Total } \\
\text { Counts } \\
\text { Radon* } \\
(b-g)\end{array}$ & $\begin{array}{c}\text { CPM } \\
\operatorname{NaI}(1,2) \\
\text { Gross } \\
(b-g)\end{array}$ & $\begin{array}{c}\text { CPM } \\
\operatorname{NaI}(1,2) \\
31 \mathrm{keV} \\
(\mathrm{b}-\mathrm{g})\end{array}$ & $\begin{array}{c}\mathrm{CPM} \\
\mathrm{NaI}(1,2) \\
81 \mathrm{keV} \\
(\mathrm{b}-\mathrm{g})\end{array}$ & $\begin{array}{l}\text { CPM } \\
\text { Total } \\
\text { Rn } \\
(b-g)\end{array}$ & $\begin{array}{r}\text { Flag } \\
\text { No. }\end{array}$ \\
\hline 8-Sep-97 & 251 & 2300 & 944 & 2 & 3555.0 & 3391.0 & 816.0 & 2096.0 & 2728.0 & 7.36 & .86 & 2.22 & 2.89 & \\
\hline 9-Sep-97 & 252 & 0600 & 944 & 3 & 2258.0 & 2145.0 & 628.0 & 1308.0 & 1626.0 & 4.66 & .67 & 1.39 & 1.72 & \\
\hline $9-$ Sep- 97 & 252 & 1400 & 944 & 4 & 2691.0 & 2611.0 & 613.0 & 1553.0 & 2091.0 & 5.62 & .65 & 1.65 & 2.22 & \\
\hline 9-Sep-97 & 252 & 2300 & 1044 & 1 & 1671.0 & 1644.0 & 380.0 & 1027.0 & 1164.0 & 3.18 & .36 & .98 & 1.11 & 1 \\
\hline 10-Sep-97 & 253 & 0600 & 1323 & 3 & 4336.0 & 4329.0 & 867.0 & 2570.0 & 3716.0 & 6.55 & .66 & 1.94 & 2.81 & 1 \\
\hline 10-Sep-97 & 253 & 1400 & 1424 & 2 & 3889.0 & 3771.0 & 680.0 & 1437.0 & 1945.0 & 5.38 & .48 & 1.01 & 1.37 & 1 \\
\hline 10-Sep-97 & 253 & 2300 & 944 & 4 & 3067.0 & 3128.0 & 551.0 & 1765.0 & 2605.0 & 6.56 & .58 & 1.87 & 2.76 & 1 \\
\hline $11-$ Sep-97 & 254 & 0600 & 944 & 1 & 963.0 & 966.0 & 335.0 & 489.0 & 706.0 & 2.04 & .35 & .52 & .75 & 1 \\
\hline 11-Sep-97 & 254 & 1400 & 944 & 2 & 1943.0 & 2124.0 & 533.0 & 1148.0 & 1545.0 & 4.31 & .56 & 1.22 & 1.64 & 1 \\
\hline 24-Sep-97 & 267 & 0213 & 960 & 1 & 7259.0 & 6970.0 & 1094.0 & 4543.0 & 6456.0 & 14.82 & 1.14 & 4.73 & 6.73 & \\
\hline 24-Sep-97 & 267 & 0213 & 960 & 2 & 432.0 & 466.0 & 114.0 & 146.0 & 236.0 & .94 & .12 & .15 & .25 & \\
\hline 24-Sep-97 & 267 & 0213 & 960 & 3 & 579.0 & 458.0 & 124.0 & 189.0 & 251.0 & 1.08 & .13 & .20 & .26 & \\
\hline 24-Sep-97 & 267 & 0213 & 960 & 4 & 1191.0 & 1237.0 & 234.0 & 667.0 & 857.0 & 2.53 & .24 & .69 & .89 & \\
\hline 11 -Nov- 97 & 315 & 1000 & 944 & 1 & 560.0 & 569.0 & 60.0 & 209.0 & 541.0 & 1.20 & .06 & .22 & .57 & \\
\hline $11-$ Nov-97 & 315 & 1800 & 944 & 2 & 1415.0 & 1335.0 & 113.0 & 642.0 & 1185.0 & 2.91 & .12 & .68 & 1.26 & \\
\hline 12 -Nov- 97 & 316 & 0200 & 944 & 3 & 1108.0 & 1060.0 & 93.0 & 480.0 & 974.0 & 2.30 & .10 & .51 & 1.03 & \\
\hline $12-$ Nov-97 & 316 & 1000 & 944 & 4 & 1803.0 & 1778.0 & 140.0 & 936.0 & 1621.0 & 3.79 & .15 & .99 & 1.72 & \\
\hline
\end{tabular}




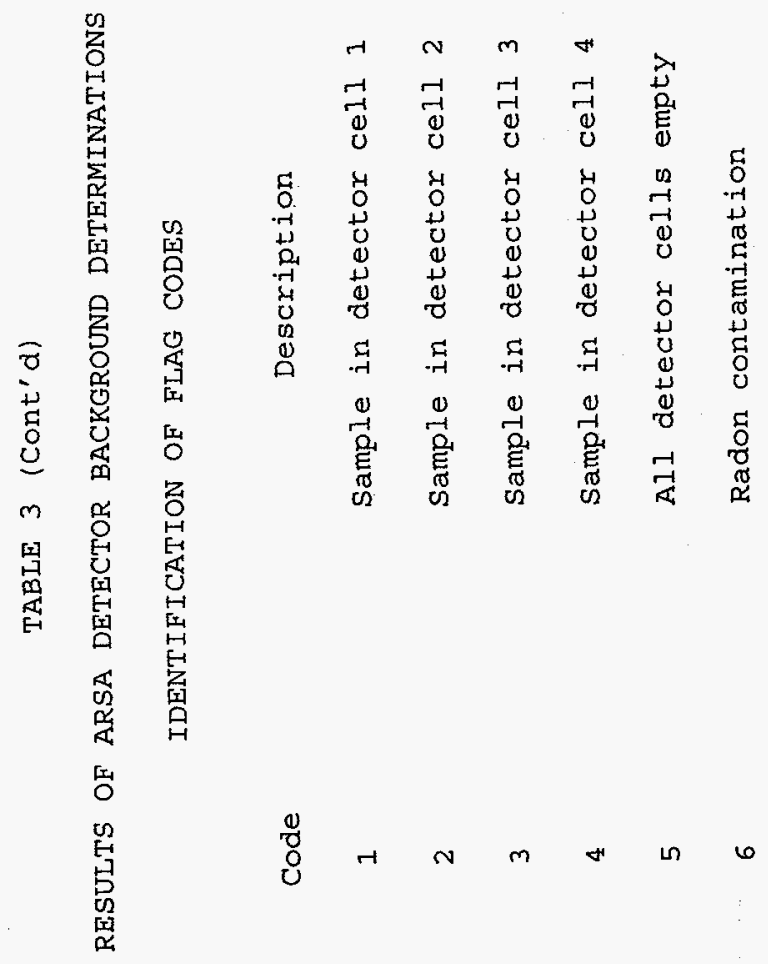




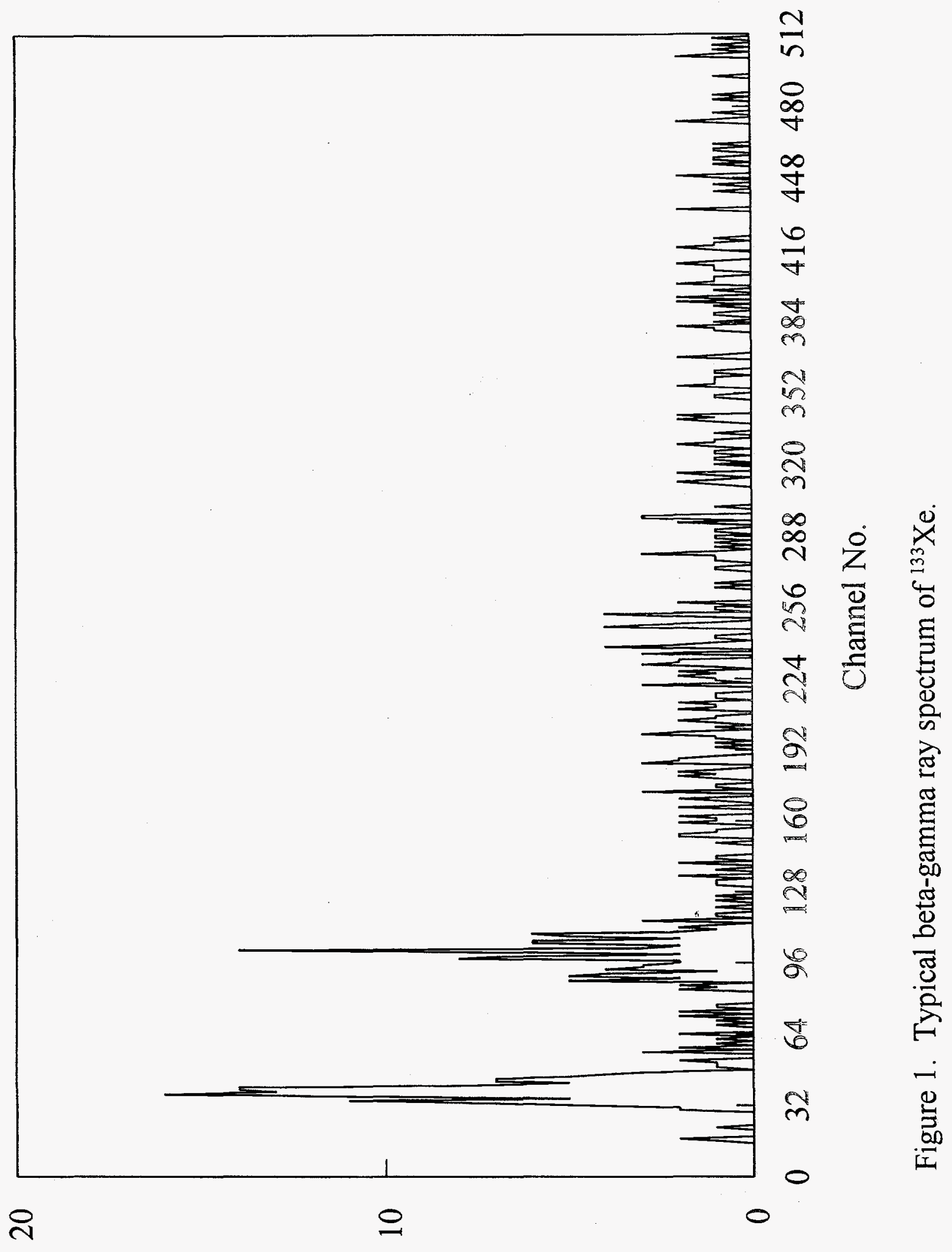

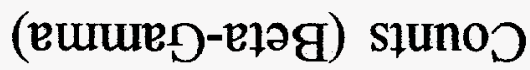




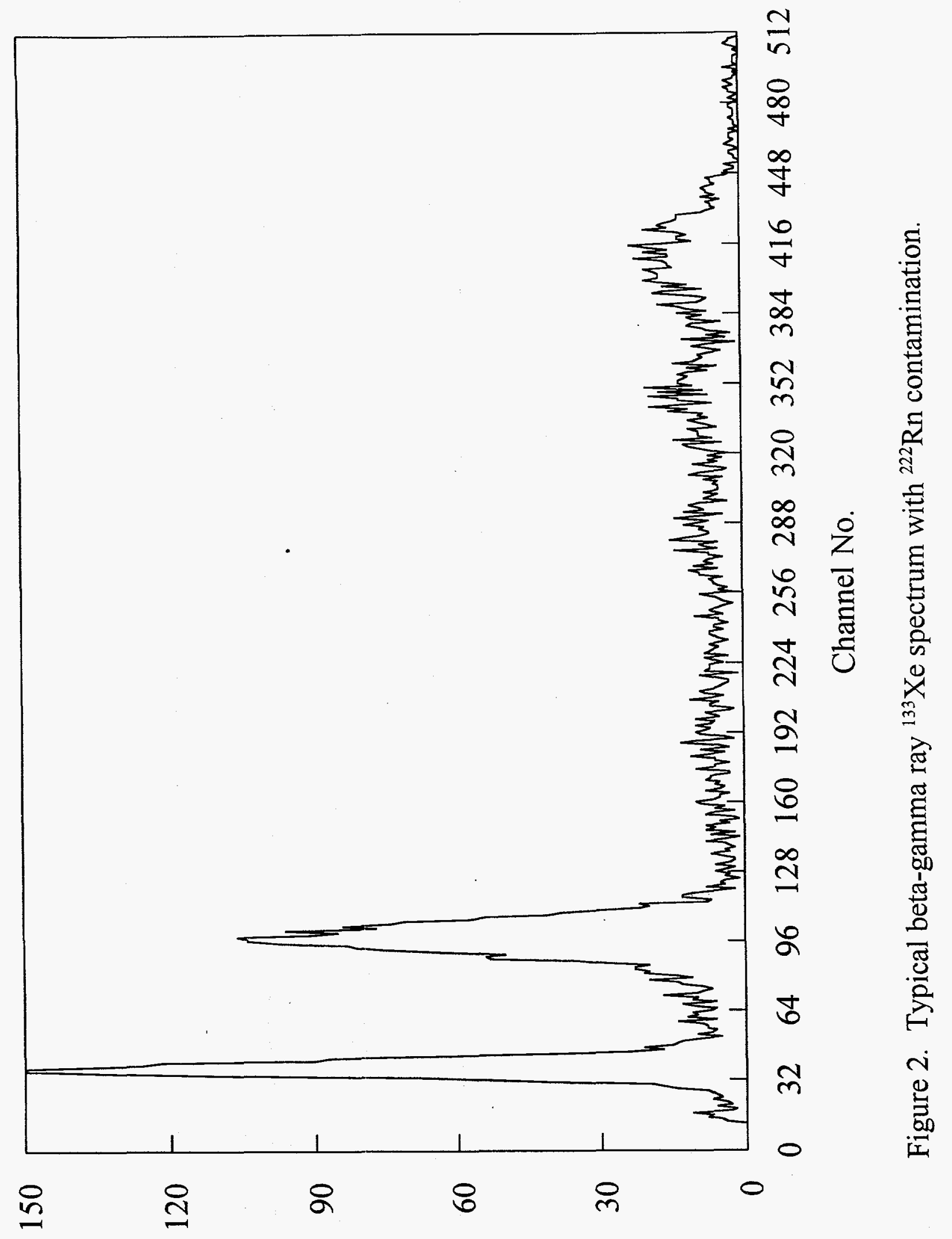

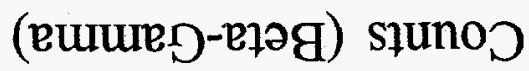




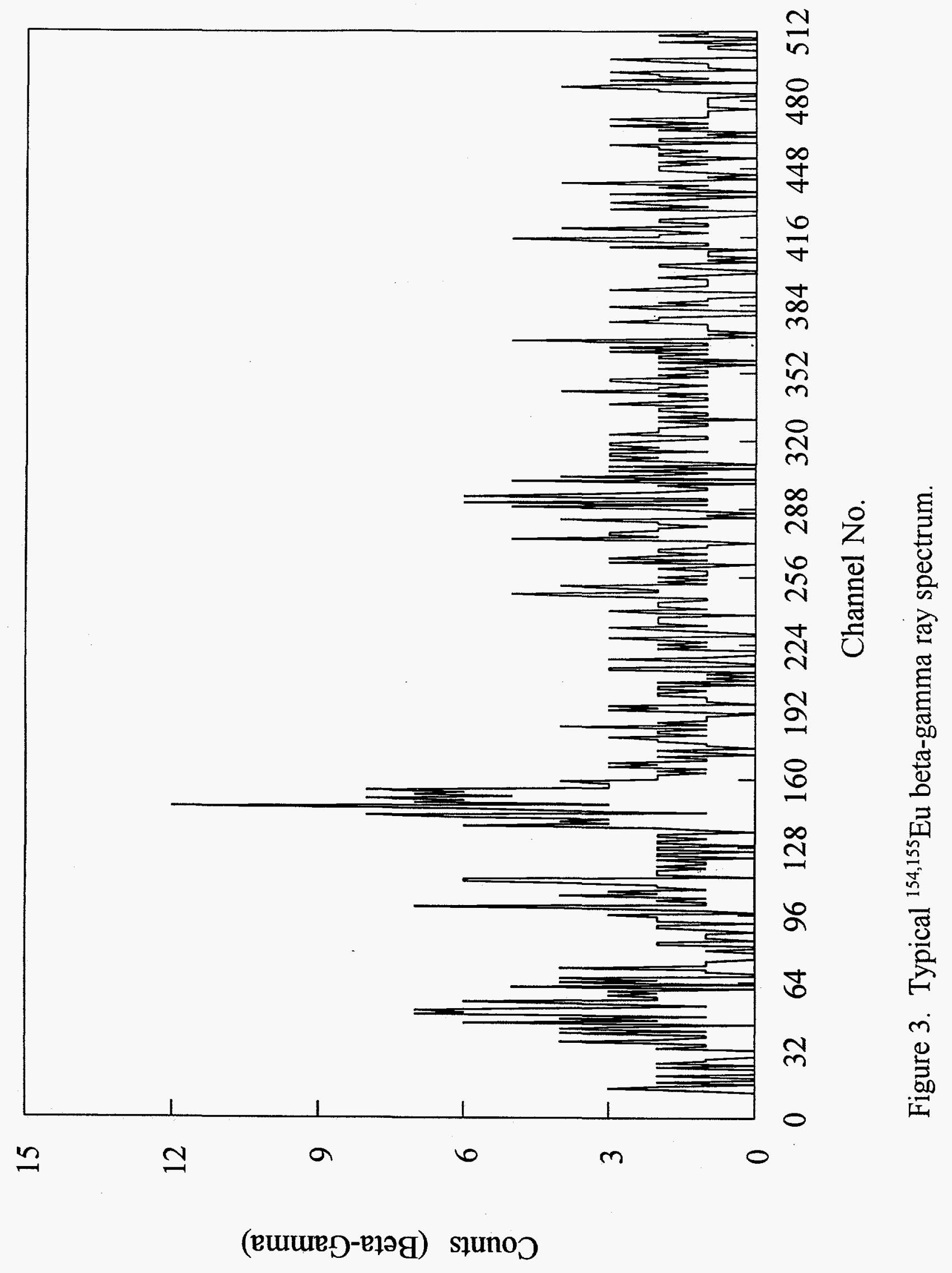




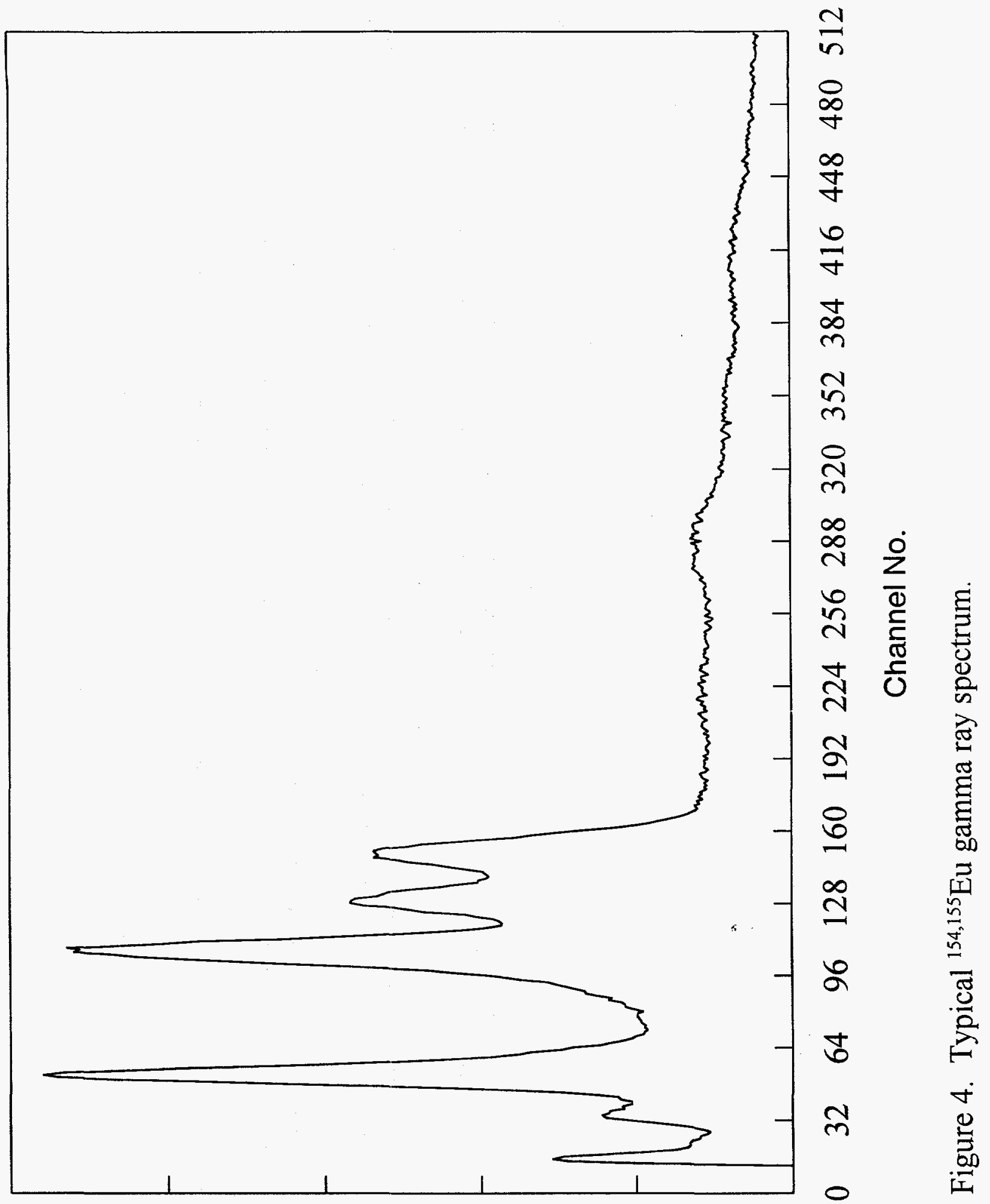

(emureg) słunoכ 


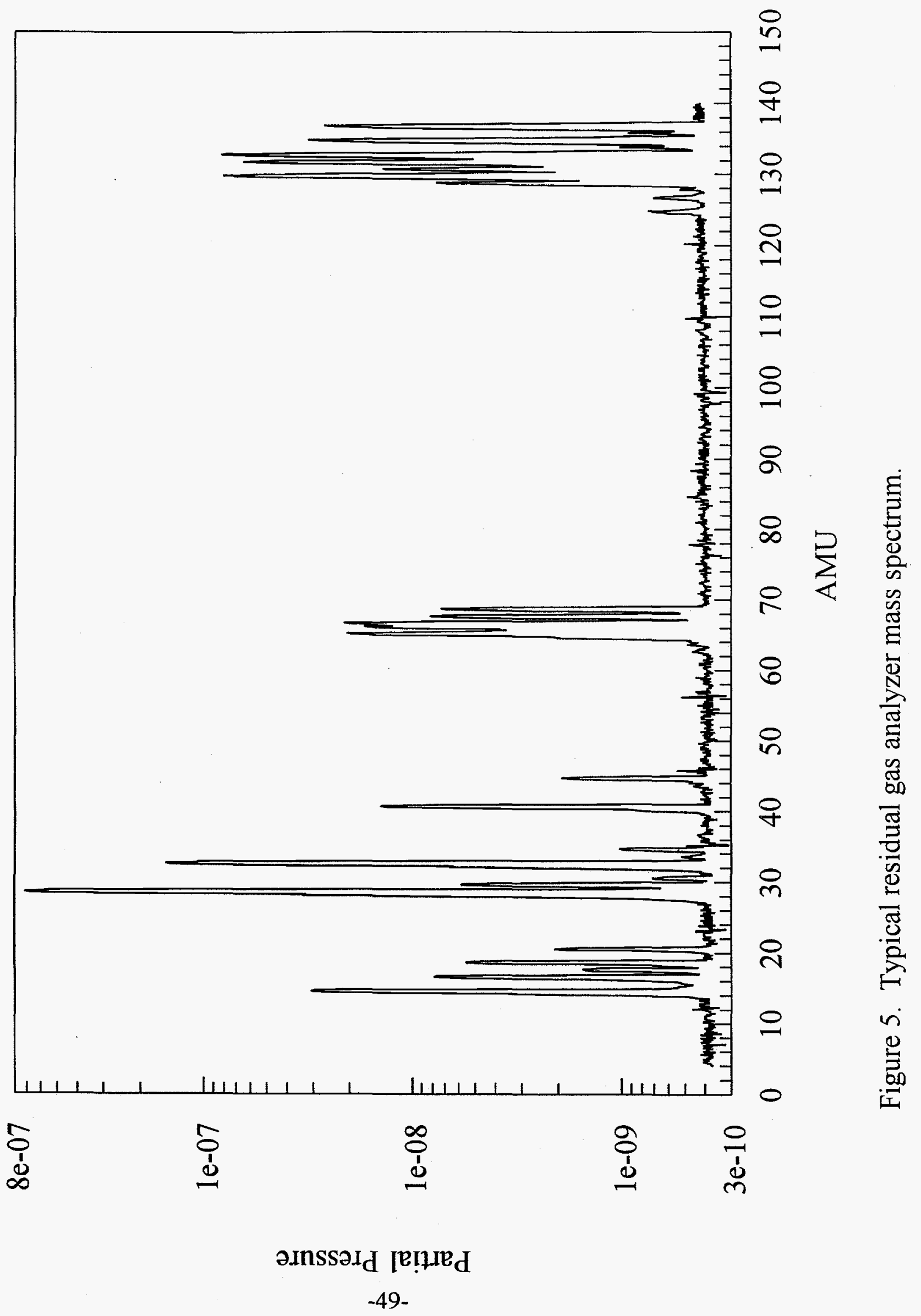




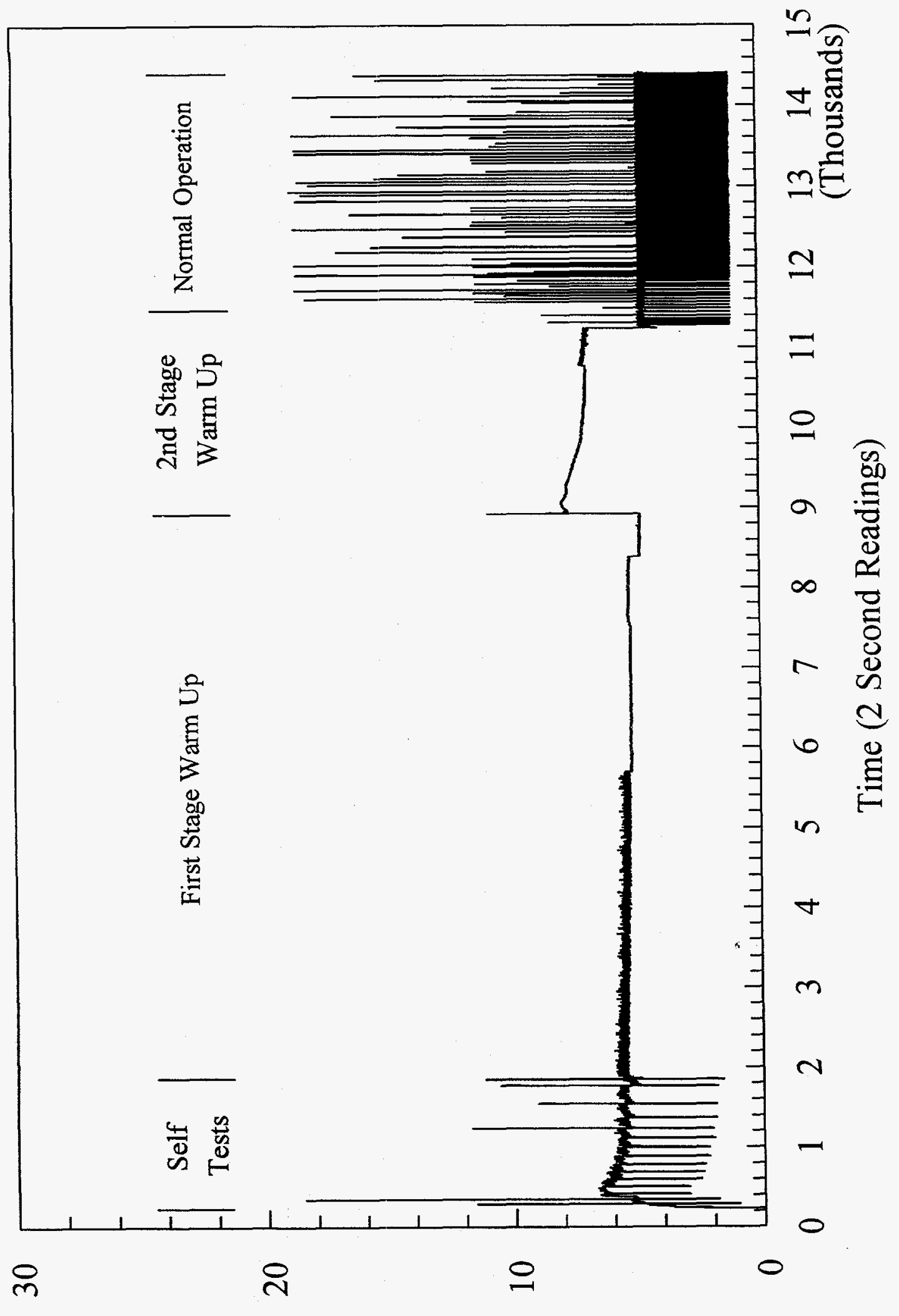

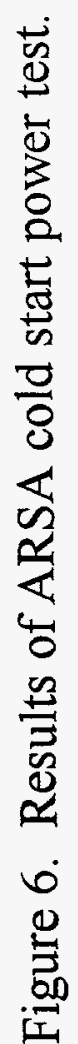

(MY) IəMO $_{\mathrm{d}}$ snoəuejueısuI 


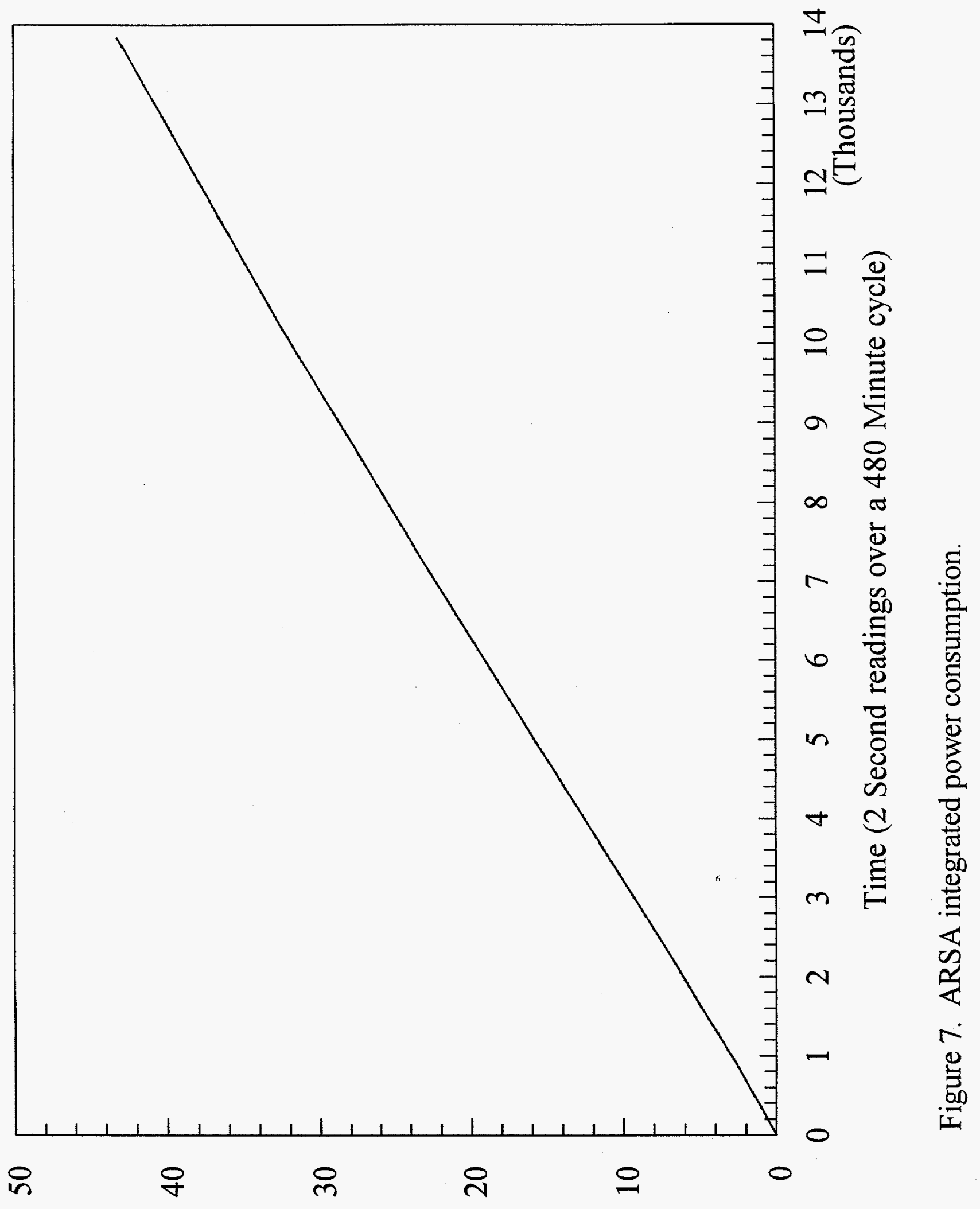

(ЧМY) ${ }^{2}{ }^{2}$ 


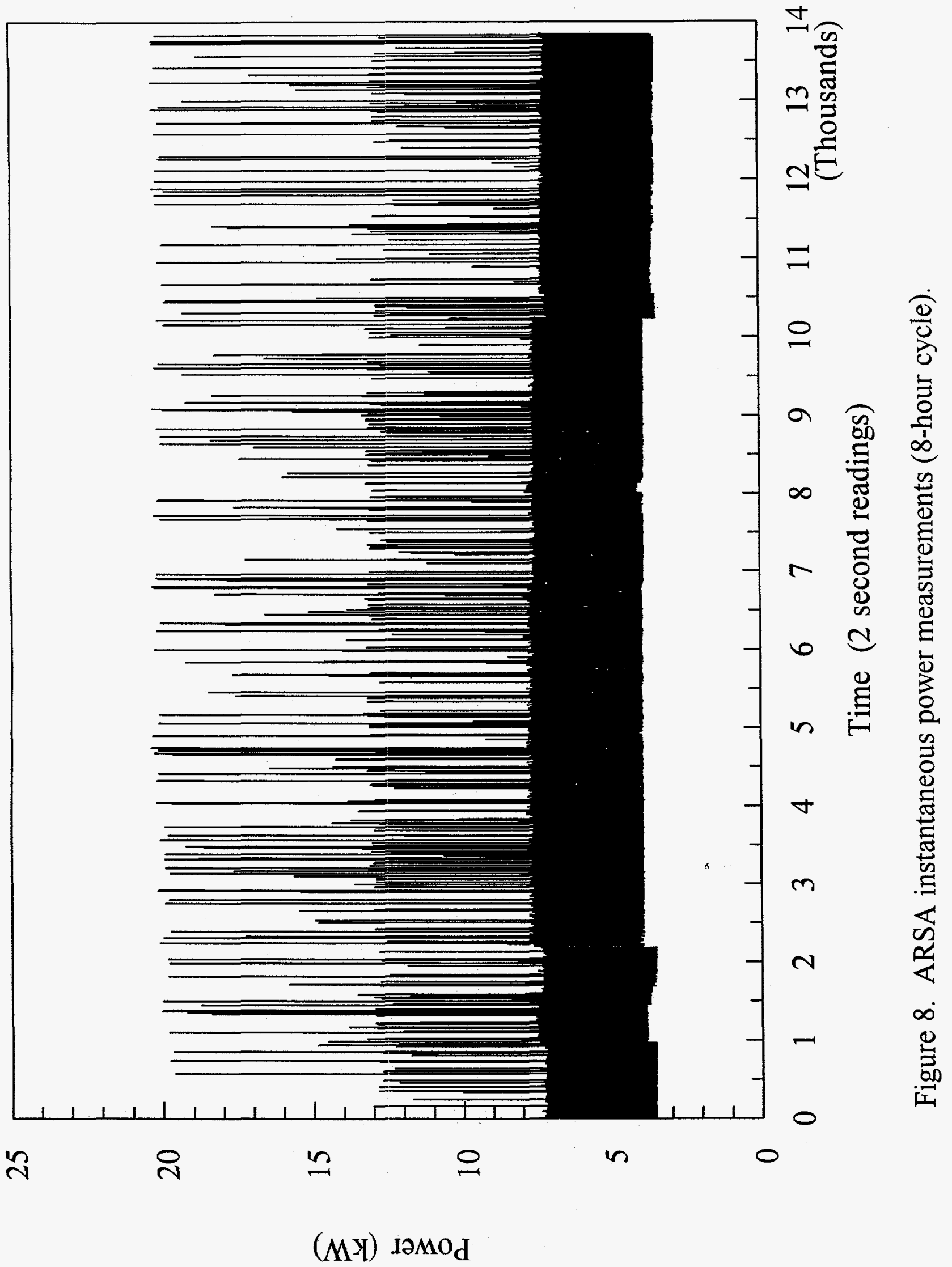


疍

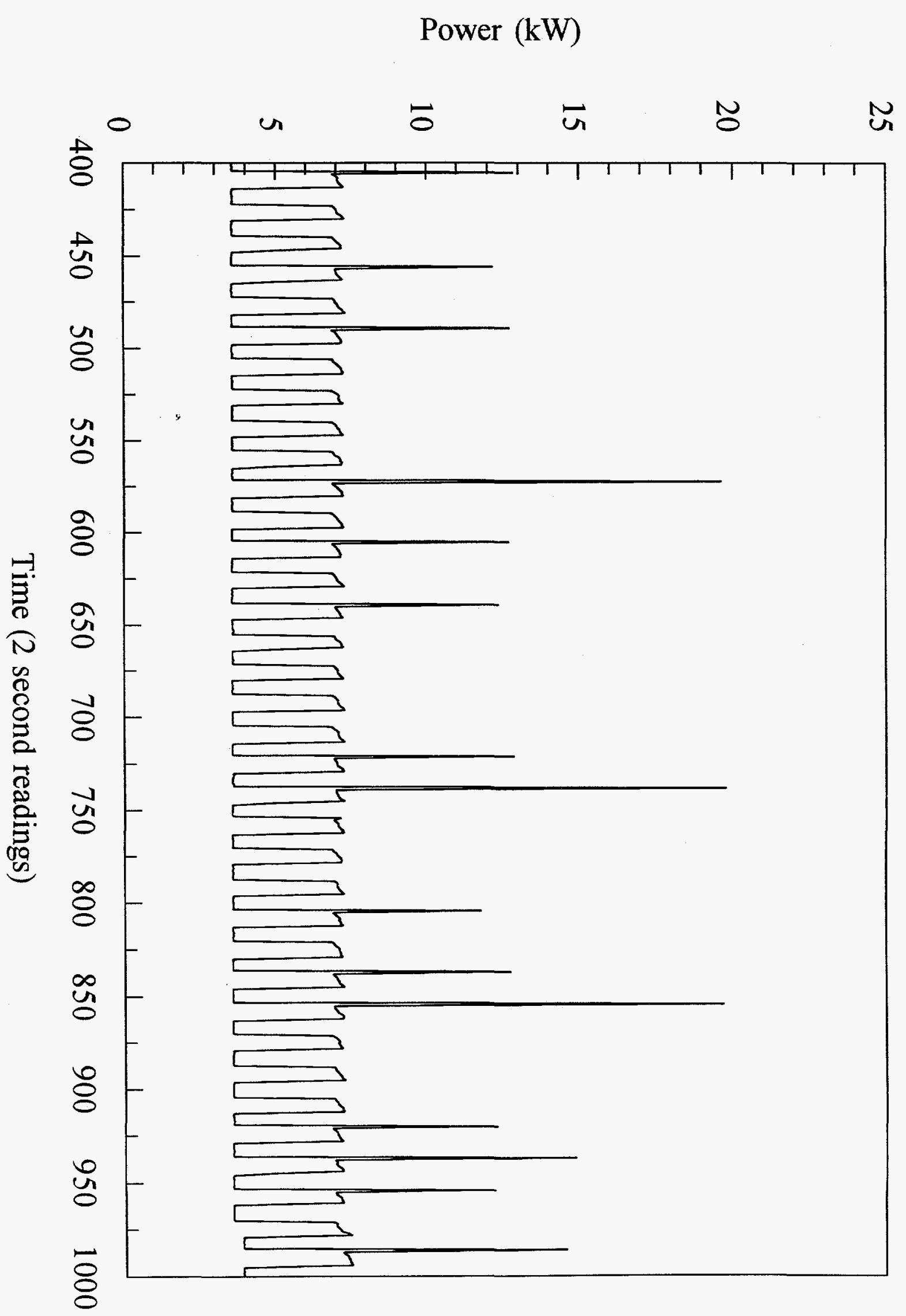




\section{APPENDIX A}

\section{RESULTS OF THE INDIVIDUAL FIELD TESTS}




\section{(AT1) - ON-SITE MEASUREMENT}

Date: May 5, 1997

Objective:

The system should be capable of on-site radioxenon separation and measurement.

\section{Required Materials:}

Multichannel analyzer (MCA) and residual gas analyzer (RGA) files from the graphical user interface.

\section{Method/Procedure:}

Access MCA and RGA files. Confirm the presence of ${ }^{133} \mathrm{Xe},{ }^{135} \mathrm{Xe}$, and radon (if any) peaks in the files integrate the appropriate beta-gamma ${ }^{133} \mathrm{Xe}$ coincidence peaks and convert to absolute recoveries from the RGA spectrum. Compare the ARSA, RGA recoveries with the results obtained from BOC analysis of archive bottles.

\section{Observation:}

From 4/21/97-4/24/97, ARSA made normal collections. EML inspected the MCA and RGA files and observed low xenon yields. On 4/24/97, the RGA file for the previous day's sample showed signs of ${ }^{135} \mathrm{Xe}$. On $4 / 25 / 97$, a software problem related to the RGA caused the syslem to shutdown. Other additional problems prevented the system from operating normally until 4/30/97. Upon inspection of the RGA files for the 4/30-5/2 collections, EML noticed considerable radon in the spectrum for all except 0502970402.rga 1. The associated background spectrums also showed the presence of radon.

Until $5 / 2 / 97$, only the total counts over the entire energy range were obtained because the software to convert to ${ }^{133} \mathrm{Xe}$ concentrations had not been made available. Additionally, the determination of elemental xenon recoveries via the RGA continued to disagree with the BOC analytical results. On the afternoon of 5/2, EML received word from PNNL that they developed and implemented a correct algorithm into the software. This could not be tested because of overheating and system shutdown on $5 / 3$.

After reinstallation of the ARSA on July 22, elemental xenon recoveries were less than anticipated and disagreed with laboratory analysis results. On Aug. $14^{\text {th }}$, the RGA was relocated from the top of the electronics cabinet to the floor and the system pneumatics were modified. Xenon recoveries improved and agreed with laboratory analysis results by a constant ratio. 
Although the software is capable of calculating radioxenon concentrations from the beta-gamma spectra, it could not be utilized during the entire test period (it lacked proper calculation parameters). 


\section{(AT2) - BACKGROUND MEASUREMENTS}

Date: April 22, 1997

\section{Objective:}

Determine that the system is performing the required background measurement within specifications by inspecting the transmitted and archived data.

\section{Required Materials:}

ARSA radiation detectors with associated electronics and software to store, archive, and integrate data as well as setting counting intervals.

\section{Method/Procedure:}

Determine the beta-gamma coincidence background of each detector cell for a period of $960 \mathrm{~min}$ $(16 \mathrm{~h})$. Determine the background of each $\mathrm{NaI}$ detector by integration of the entire spectrum. Sum both $\mathrm{NaI}$ backgrounds for total detector background.

\section{Observation:}

Background for each detector cell was obtained (see AT10) using the ARSA system's monitor. ARSA background data base was established (see Table 4). Spectral files also examined at EML's remote computer terminal.

\section{Results:}

All values agree with those obtained with ARSA system's monitor. Spectral files have not been transmitted via RS-232 (modem). The latter was deleted from the test plan.

Method calls for counting the sample in the cell with simultaneous background measurement in paired cell. However, counting times for paired cell did not agree (20 min difference). There was a 16 min difference after ARSA reinstallation.

Observed an increase in background from residual radioxenon and radon activity remaining in the detector cell after the sample has been transferred to the archive bottle and the cell evacuated under pressure (Memory Effect). 


\begin{tabular}{ccccccc}
\hline File Name & $\begin{array}{c}\text { Cell } \\
\text { No. }\end{array}$ & $\begin{array}{c}\text { Sample } \\
\text { Type }\end{array}$ & $\begin{array}{c}\mathrm{cpm} 31 \mathrm{keV} \\
\left({ }^{133} \mathrm{Xe}\right)\end{array}$ & $\mathrm{cpm} \mathrm{Rn}^{(1)}$ & $\%$ Res. $\mathrm{Xe}^{(2)}$ & $\%$ Res. $\mathrm{Rn}^{(2)}$ \\
\hline 0831970641.Bkg_2 & 2 & Bkg. 1 & 0.221 & 1.312 & - & - \\
0831972241.Sam_2 & 2 & Sample & 8.823 & 7.108 & - & - \\
0901971441.Bkg_2 & 2 & Bkg. 2 & 0.540 & 1.552 & 3.71 & 4.42 \\
0830970641.Bkg_3 & 3 & Bkg. 1 & 0.203 & 0.554 & - & - \\
0830972241.Sam_3 & 3 & Sample & 1.940 & 4.219 & - & - \\
0831971441.Bkg_3 & 3 & Bkg. 2 & 0.255 & 0.960 & 3.00 & 11.10 \\
083971441.Bkg_4 & 4 & Bkg. 1 & 0.124 & 0.709 & - & - \\
0831970641.Sam_4 & 4 & Sample & 20.797 & 6.108 & - & - \\
0831972241.Bkg_4 & 4 & Bkg. 2 & 0.958 & 1.274 & 4.03 & 10.4 \\
\hline
\end{tabular}

Radon residual activity in cells (see Test AT7, Radon, Third Test Results).

\begin{tabular}{cclcc}
\hline Sample Date & Cell No. & Sample Type & ${\text { cpm } \mathrm{Rn}^{(1)}}$ & \% Res. $\mathrm{Rn}^{(2)}$ \\
\hline $10 / 29 / 97$ & 2 & Bkg. 1 & 0.69 & - \\
$10 / 30 / 97$ & 2 & Sample & 21.50 & - \\
$10 / 30 / 97$ & 2 & Bkg. 2 & 3.47 & 13.4 \\
$10 / 30 / 97$ & 3 & Bkg. 1 & 2.84 & - \\
$10 / 30 / 97$ & 3 & Sample & 18.90 & - \\
$10 / 30 / 97$ & 3 & Bkg. 2 & 4.90 & 13.7 \\
$10 / 30 / 97$ & 4 & Bkg. 1 & 0.44 & - \\
$10 / 30 / 97$ & 4 & Sample & 829.00 & - \\
$10 / 30 / 97$ & 4 & Bkg. 2 & 108.00 & 13.7 \\
\hline
\end{tabular}

(1) Sum of the $214 \mathrm{~Pb}(242,295$ and $352 \mathrm{keV})$ gamma peaks.

(2) \% residual activity (Memory Effect) $=($ cpm Bkg. 2 - cpm Bkg. 1)/(cpm sample - cpm Bkg. 1) x 100 . 


\section{AT3 ENERGY CALIBRATIONS}

Date: April 17, 1997

\section{Objective:}

Determine that the system is performing the required energy/channel measurement and calculations within specifications by inspecting the transmitted and archived data files.

Required Materials:

${ }^{154,155} \mathrm{Eu}$ calibrations, ARSA radiation detectors with associated electronics and software.

\section{Method/Procedure:}

Count the ${ }^{154,155} \mathrm{Eu}$ calibration source in each cell for a period of $16 \mathrm{~h}$. Examine the digitized gamma ray spectra for each $\mathrm{NaI}$ detector to determine the channel number associated with the ${ }^{154,155} \mathrm{Eu}, 42$ $\mathrm{keV}, 86.5 \mathrm{keV}, 105 \mathrm{keV}$ and $123 \mathrm{keV}$ energies. Calculate the $\mathrm{keV} /$ channel for each $\mathrm{NaI}$ detector. All of the data were obtained from cross-talk experimental (AT9) spectra. 


\section{Observation:}

\begin{tabular}{|c|c|c|c|c|}
\hline \multirow{2}{*}{$\begin{array}{c}\text { Detector CELL } \\
\text { No. }\end{array}$} & \multicolumn{2}{|c|}{$\mathrm{NaI}(1)$} & \multicolumn{2}{|c|}{$\mathrm{NaI}(2)$} \\
\hline & Channel No. & Energy $(\mathrm{keV})$ & Channel No. & Energy (keV) \\
\hline 1 & 54 & 42 & 51 & 42 \\
\hline \multirow[t]{4}{*}{$(4 / 11 / 97)$} & 110 & 86.5 & 104 & 86.5 \\
\hline & 132 & 105 & 126 & 105 \\
\hline & 154 & 123 & 146 & 123 \\
\hline & \multicolumn{2}{|c|}{$81 / 100=0.81 \mathrm{keV} /$ channel } & \multicolumn{2}{|c|}{$81 / 95=0.85 \mathrm{keV} /$ channel } \\
\hline 2 & 52 & 42 & 49 & 42 \\
\hline \multirow[t]{4}{*}{$(4 / 14 / 97)$} & 108 & 86.5 & 102 & 86.5 \\
\hline & 128 & 105 & 123 & 105 \\
\hline & 151 & 123 & 143 & 123 \\
\hline & \multicolumn{2}{|c|}{$81 / 99=0.82 \mathrm{keV} /$ channel } & \multicolumn{2}{|c|}{$81 / 94=0.86 \mathrm{keV} /$ channel } \\
\hline 3 & 50 & 42 & 50 & 42 \\
\hline \multirow[t]{4}{*}{$(4 / 17 / 97)$} & 104 & 86.5 & 102 & 86.5 \\
\hline & 124 & 105 & 124 & 105 \\
\hline & 144 & 123 & 143 & 123 \\
\hline & \multicolumn{2}{|c|}{$81 / 94=0.86 \mathrm{keV} /$ channel } & \multicolumn{2}{|c|}{$81 / 93=0.87 \mathrm{keV} /$ channel } \\
\hline 4 & 53 & 42 & 53 & 42 \\
\hline \multirow[t]{4}{*}{$(4 / 18 / 97)$} & 109 & 86.5 & 108 & 86.5 \\
\hline & 131 & 105 & 130 & 105 \\
\hline & 152 & 123 & 151 & 123 \\
\hline & \multicolumn{2}{|c|}{$81 / 99=0.82 \mathrm{keV} /$ channel } & \multicolumn{2}{|c|}{$81 / 98=0.83^{5} \mathrm{keV} /$ channel } \\
\hline
\end{tabular}

Note: All of the data are from the ARSA system monitor. The results were verified with EML's remote monitor. File transmission via RS-232 modem was deleted from the test plan. 


\section{(AT4) - GAMMA RAY SPECTRUM RANGE}

Date: April 17, 1997

\section{Objective:}

Determine that the system is performing the required measurements within the specified energy range by inspecting transmitted and archived data files.

\section{Required Materials:}

${ }^{154,155} \mathrm{Eu}$ calibration sources. ARSA detector cells and associated electronics, data acquisition and reduction software.

\section{Method/Procedure:}

Count a ${ }^{154,155} \mathrm{Eu}$ calibration source in each cell. Determine the gamma-ray energy range.

\section{Observation:}

Energy range as determined from AT10 is $15 \mathrm{keV}$ to $440 \mathrm{keV}$. Test requirement is $20 \mathrm{keV}$ to $512 \mathrm{keV}$. Results verified via EML remote monitor but not the transmitted data files because an RS-232 connection had not been established. The RS-232 evaluation was deleted from the test plan. 
Date: December 1, 1997

Objective:

The system shall be able to vary count time of sample.

Required Materials:

ARSA, ARSA software and monitor.

Method/Procedure:

Remotely retrieve spectra without interfering with the counting time at $1,4,10$ and $15 \mathrm{~h}$, and verify proper operation by inspecting data files.

\section{Observation:}

Retrieved spectra can not be accessed without interfering with the counting time. ARSA counting must be terminated, the spectral file downloaded from the ARSA computer to the PC, and counting restarted at time zero. All saved files must be summed for quantitation. 


\section{(AT6) - EFFICIENCY CALIBRATION}

Date: September 29, 1997

\section{Objectives:}

a. The system will have a means of remotely measuring relative detector efficiency.

b. The system will contain standards to determine the detector's stability.

\section{Required Materials:}

${ }^{154,155} \mathrm{Eu}(0.5 \mathrm{nCi})$ counting standards.

\section{Method/Procedure:}

a. Remotely measure the relative detector efficiency using PNNL methodolgy. Determine that the system is performing the efficiency measurement and calculations by inspecting the files.

b. Determine the reproducibility of the counting standards by determining the detector backgrounds $(16 \mathrm{~h})$ followed by the introduction of the Europium counting standards into the detectors via the ARSA source transfer system. Count the standards for $16 \mathrm{~h}$. Withdraw the secondary standards from the detectors and repeat the background and secondary standard determinations over two additional cycles.

Observation:

a. Detector efficiencies determined prior to shipment to sampling site. Detector stability was determined with Europium sources. No software for performing remote efficiency calculations.

b. All counting data represents the sum of both detector channels, NaI (1) and (2), over the entire energy range ( 512 channels) with background subtracted (any beta-gamma window). 


\begin{tabular}{lcccc}
\hline Detector No. & CELL 1 & CELL 2 & CELL 3 & CELL 4 \\
\hline $\begin{array}{l}\text { Total Counts } \\
(9 / 24 / 97)\end{array}$ & 5903 & 5114 & 2330 & 7125 \\
$\begin{array}{l}\text { Total Counts } \\
(9 / 25 / 97)\end{array}$ & 5646 & 5405 & 2372 & 7537 \\
$\begin{array}{l}\text { Total Counts } \\
(9 / 27 / 97)\end{array}$ & 5917 & 5388 & 2369 & 7389 \\
$\begin{array}{l}\text { Mean } \\
\pm \text { Std. Dev. }\end{array}$ & 5822 & 5302 & 2357 & 7350 \\
$(\%$ SD) & $152(2.6 \%)$ & $163(3.1 \%)$ & $23(1.0 \%)$ & $209(2.9 \%)$ \\
\hline
\end{tabular}




\section{(AT7) RADON}

Date: September 30, 1997

\section{Objective:}

The system shall have a way to reduce radon contamination so it does not interfere with the measurement of the isotopes of interest.

\section{Required Materials:}

EML Environmental Test Chamber, ${ }^{222} \mathrm{Rn}$ source.

\section{Method/Procedure:}

Determine that the system is performing the required reduction of radon interference at twice the highest recorded atmospheric value as determined by a literature search ( $3 \mathrm{pCi})$. Evaluate sample spectral data to determine if radon breakthrough occurs. PNNL personnel should be present during this test.

First Test: Run for one 8-h cycle only @ $3 \mathrm{pCi} / \mathrm{L}{ }^{222} \mathrm{Rn}$ chamber concentration.

Second Test: Same as first test.

Third Test: Run for three 8-h cycles @ $3.8 \pm 0.2 \mathrm{pCi} / \mathrm{L}^{222} \mathrm{Rn}$ chamber concentration. 


\section{Observation:}

\section{First Test:}

Note: Leak in sample air intake manifold - $20 \%$ of chamber air sampled.

\begin{tabular}{ccccc}
\hline $\begin{array}{c}\text { Sample Date } \\
(1997)\end{array}$ & Sample Type & Gross Count* & $\begin{array}{c}\text { Count Time } \\
(\mathrm{m})\end{array}$ & $\begin{array}{c}\text { Total Rn** } \\
(\mathrm{cpm})\end{array}$ \\
\hline $9 / 8$ & Pre-sample Bkg & 944 & 944 & 1.76 \\
$9 / 9$ & Previous Sample & 8969 & 960 & 9.34 \\
$9 / 9$ & $\begin{array}{c}\text { Rn Spiked } \\
\text { Sample }\end{array}$ & 18807 & 1061 & 17.0 \\
& Post-sample & & 1323 & 2.95 \\
$9 / 11$ & Bkg & 3898 & & \\
\hline
\end{tabular}

*Sum of the ${ }^{214} \mathrm{~Pb}(242,295$ and $352 \mathrm{keV})$ peaks.

** Radon sample activity increased by a factor of 1.5 to 2.0 .

Second Test - sample air intake leak repaired:

\begin{tabular}{cccccc}
\hline $\begin{array}{c}\text { Sample } \\
\text { Date (1997) }\end{array}$ & $\begin{array}{c}\text { Sample } \\
\text { Type }\end{array}$ & $\begin{array}{c}\text { Gross } \\
\text { Count* }\end{array}$ & $\begin{array}{c}\text { Count Time } \\
(\mathrm{min})\end{array}$ & $\begin{array}{c}\text { Detector } \\
\text { No. }\end{array}$ & $\begin{array}{c}\text { Total Rn** } \\
(\mathrm{cpm})\end{array}$ \\
\hline $10 / 23$ & $\begin{array}{c}\text { Pre-sample } \\
\text { Bkg }\end{array}$ & 331 & 960 & 1 & 0.34 \\
$10 / 24$ & Rn Sample & 810 & 960 & 1 & 0.84 \\
$10 / 25$ & $\begin{array}{c}\text { Post-sample } \\
\text { Bkg }\end{array}$ & 464 & 960 & 1 & 0.48 \\
$10 / 25$ & $\begin{array}{c}\text { Sample* } \\
\text { Post-sample } \\
\text { Bkg* }\end{array}$ & 191000 & 1020 & 3 & 187.00 \\
& 25100 & 944 & 3 & 26.60 \\
\hline
\end{tabular}

* Represents the next sample processed through the same purification channel as the radon test sample.

**Sum of the ${ }^{214} \mathrm{~Pb}(242,295$ and $352 \mathrm{keV})$ peaks. 


\begin{tabular}{|c|c|c|c|c|c|c|}
\hline $\begin{array}{c}\text { Sample } \\
\text { Date } \\
(1997)\end{array}$ & $\begin{array}{c}\text { Sample } \\
\text { Type }\end{array}$ & $\begin{array}{c}\text { Detector } \\
\text { No. }\end{array}$ & $\begin{array}{l}\text { Purification } \\
\text { Stream No. }\end{array}$ & $\begin{array}{c}\text { Gross } \\
\text { Count* }\end{array}$ & $\begin{array}{l}\text { Count } \\
\text { Time } \\
\text { (min) }\end{array}$ & $\begin{array}{c}\text { Total Rn** } \\
(\mathrm{cpm})\end{array}$ \\
\hline $10 / 28$ & Pre-sample Bkg & 4 & - & 488 & 943 & 0.52 \\
\hline $10 / 29$ & Rn Sample & 4 & 0 & 1372 & 960 & 1.43 \\
\hline $10 / 31$ & Post-sample Bkg & 4 & - & 411 & 944 & 0.44 \\
\hline $10 / 29$ & Pre-sample Bkg & 1 & - & 367 & 944 & 0.39 \\
\hline $10 / 29$ & Rn Sample & 1 & 1 & 15535 & 960 & 16.2 \\
\hline $10 / 29$ & Post-sample Bkg & 1 & - & 2347 & 944 & 2.48 \\
\hline $10 / 29$ & Pre-sample Bkg & 2 & - & 656 & 944 & 0.69 \\
\hline $10 / 30$ & Rn Sample & 2 & 0 & 20656 & 960 & 21.5 \\
\hline $10 / 30$ & Post-sample Bkg & 2 & - & 3274 & 944 & 3.47 \\
\hline $10 / 30$ & Pre-sample Bkg & 3 & - & 2685 & 944 & 2.84 \\
\hline $10 / 31$ & Post-sample Bkg & 3 & - & 4629 & 944 & 4.90 \\
\hline $10 / 30$ & Pre-sample Bkg & 4 & - & 411 & 944 & 0.44 \\
\hline $10 / 30$ & Atmos. Sample & 4 & 0 & 796025 & 960 & 829 \\
\hline $10 / 30$ & Post-sample Bkg & 4 & - & 102332 & 945 & 108 \\
\hline $10 / 30$ & Pre-sample Bkg & 1 & - & 2347 & 944 & 2.49 \\
\hline $10 / 31$ & Atmos. Sample & 1 & 1 & 4908 & 964 & 5.09 \\
\hline $10 / 31$ & Post-sample Bkg & 1 & - & 1258 & 944 & 1.33 \\
\hline $10 / 30$ & Pre-sample Bkg & 2 & - & 3274 & 944 & 3.47 \\
\hline $10 / 31$ & Atmos. Sample & 2 & 0 & 3318 & 960 & 3.46 \\
\hline $11 / 01$ & Post-sample Bkg & 2 & - & 1383 & 943 & 1.47 \\
\hline
\end{tabular}

*Sum of the ${ }^{214} \mathrm{~Pb}(242,295$ and $352 \mathrm{keV})$ peaks.

**Radon retained by traps and eluted into the following sample. 


\section{(AT8) - XENON MEASUREMENT MINIMUM DETECTION LEVELS}

Date: January 26, 1998

\section{Objective:}

Determine minimum detection levels for a $6-\mathrm{h}$ cycle time.

\section{Required Materials:}

ARSA background spectra, counting efficiencies, volume of air sampled.

\section{Method/Procedure:}

Calculate the minimum detectable concentrations (MDCs) of ${ }^{131 \mathrm{~m}} \mathrm{Xe},{ }^{133 \mathrm{~m}} \mathrm{Xe},{ }^{133 \mathrm{~g}} \mathrm{Xe}$ and ${ }^{135} \mathrm{Xe}$ for an 8-h cycle time.

\section{Observation:}

\begin{tabular}{cccc}
\hline Radionuclide & $\begin{array}{c}\text { Specified MDC } \\
\left(\mathrm{pCi} / \mathrm{m}^{3}\right)\end{array}$ & $\begin{array}{c}\text { PNNL ARSA } \\
\mathrm{MDC}^{(1)}\left(\mathrm{pCi} / \mathrm{m}^{3}\right)\end{array}$ & $\begin{array}{c}\text { EML ARSA MDC } \\
\left(\mathrm{pCi} / \mathrm{m}^{3}\right)\end{array}$ \\
\hline${ }^{131 \mathrm{~m}} \mathrm{Xe}$ & $<0.59$ & - & $(4)$ \\
${ }^{133 \mathrm{~m}} \mathrm{Xe}$ & $<0.135$ & 0.006 & $\left({ }^{(4)}\right.$ \\
${ }^{133 \mathrm{~g}} \mathrm{Xe}$ & $<0.046$ & $0.006^{(2)}$ & $0.001^{(5)}$ \\
${ }^{135} \mathrm{Xe}$ & $<0.018$ & 0.005 & $0.001^{(5)}$ \\
\hline
\end{tabular}

(1) Reeder. P. L., T. W. Bowyer, and K. H. Abel, Analysis of Beta-Gamma Spectra for the PNNL ARSA and estimate of minimum detectable activities for $\left({ }^{131 m} \mathrm{Xe}+{ }^{133 m} \mathrm{Xe}\right),{ }^{133 \mathrm{~g}} \mathrm{Xe}$, and ${ }^{135} \mathrm{X}$ (e PNNL-11784 (1997).

(2) Mid-point of range $0.004-0.009 \mathrm{pCi} / \mathrm{m}^{3}$.

(3) HASL 300, $28^{\text {th }}$ Edition, Section 4.5.3, Vol 1 (1997).

(4) Dependent on sample ${ }^{133} \mathrm{Xe}$ and ${ }^{135} \mathrm{Xe}$ concentration levels.

(5) Determined from background runs obtained at the beginning of the test period (April 1997). For samples containing ${ }^{222} \mathrm{Rn}$ contamination, the $\mathrm{MDC}$ is $0.006 \mathrm{pCi} / \mathrm{m}^{3}$ (determined from detector background obtained after August 15, 1997). 


\section{AT9 (ADDED) DETECTOR CROSS-TALK}

Date: April 17, 1997

\section{Objective:}

Determine the background and contribution to the background (cross-talk) from the internal calibration source for each detector.

\section{Required Materials:}

${ }^{154,155}$ Eu calibration sources, ARSA radiation detectors and associated electronics.

\section{Method/Procedure:}

Determine the background for each of the four detector cells for a $16-\mathrm{h}$ counting period. After establishing the background counts for each detector cell, program the system so that the calibration $\left({ }^{154,155} \mathrm{Eu}\right)$ source is introduced into the first detector cell. Determine the number of counts in each detector cell over 16-h. Introduce the calibration source into cell \#2 and count for 16-h. Repeat for cell \#3 and \#4. Record the total counts over the entire energy range for each beta scintillation cell. Calculate the percentage contribution from the source to each empty cell.

\section{Observation:}

Backgrounds for 4 cells taken on 4/10/97@0739 (16 h and $22 \mathrm{~s})$.

\begin{tabular}{|c|c|c|c|c|}
\hline & $\begin{array}{c}\text { CELL } 1 \\
\text { (counts } \\
\beta-\gamma)\end{array}$ & $\begin{array}{c}\text { CELL } 2 \\
\text { (counts } \\
\beta-\gamma)\end{array}$ & $\begin{array}{c}\text { CELL } 3 \\
\text { (counts } \\
\beta-\gamma)\end{array}$ & $\begin{array}{c}\text { CELL } 4 \\
\text { (counts } \\
\beta-\gamma)\end{array}$ \\
\hline $\begin{array}{r}\mathrm{NaI} \\
\text { (1) }\end{array}$ & 398 & 313 & 36 & 543 \\
\hline $\begin{array}{r}\mathrm{NaI} \\
(2)\end{array}$ & 356 & 321 & 401 & 598 \\
\hline
\end{tabular}

Counting with ${ }^{154,155} \mathrm{Eu}$ source in each cell - all counts for $16 \mathrm{~h}$ and $22 \mathrm{~s}$. 


\begin{tabular}{|c|c|c|c|c|}
\hline & $\begin{array}{c}\text { CELL 1 } \\
\text { (counts } \beta-\gamma)\end{array}$ & $\begin{array}{c}\text { CELL } 2 \\
\text { (counts } \beta-\gamma)\end{array}$ & $\begin{array}{c}\text { CELL } 3 \\
\text { (counts } \beta-\gamma)\end{array}$ & $\begin{array}{c}\text { CELL } 4 \\
\text { (counts } \beta-\gamma)\end{array}$ \\
\hline \multicolumn{5}{|c|}{ 4/11/97@1533 h } \\
\hline $\mathrm{NaI}(1)$ & 3249 (Std) & 601 & 459 & 632 \\
\hline $\mathrm{NaI}(2)$ & $2327(\mathrm{Std})$ & 543 & 447 & 604 \\
\hline \multicolumn{5}{|c|}{ 4/14/97@2132 h } \\
\hline $\mathrm{NaI}(1)$ & 885 & 2739 (Std) & 524 & 491 \\
\hline $\mathrm{NaI}(2)$ & 810 & $2732(\mathrm{Std})$ & 494 & 515 \\
\hline \multicolumn{5}{|c|}{ 4/17/97@0437 h } \\
\hline $\mathrm{NaI}(1)$ & 316 & 476 & 525 & $5012(\mathrm{Std})$ \\
\hline $\mathrm{NaI}(2)$ & 345 & 459 & 459 & $4880(\mathrm{Std})$ \\
\hline \multicolumn{5}{|c|}{ 4/17/97@2038 h } \\
\hline $\mathrm{NaI}(1)$ & 316 & 476 & 525 & $5012(\mathrm{Std})$ \\
\hline $\mathrm{NaI}(2)$ & 345 & 459 & 459 & $4880(\mathrm{Std})$ \\
\hline
\end{tabular}

Detector backgrounds 4/18/97@ 1135 h (CELL 1, added background for 0912 h) - total counts over 16 h, 22 s.

\begin{tabular}{ccccc}
\hline & $\begin{array}{c}\text { CELL 1 } \\
(\text { counts } \beta-\gamma)\end{array}$ & $\begin{array}{c}\text { CELL 2 } \\
(\text { counts } \beta-\gamma)\end{array}$ & $\begin{array}{c}\text { CELL 3 } \\
(\text { counts } \beta-\gamma)\end{array}$ & $\begin{array}{c}\text { CELL 4 } \\
(\text { counts } \beta-\gamma)\end{array}$ \\
\hline NaI (1) & 225 & 262 & 325 & 402 \\
NaI (2) & 252 & 241 & 300 & 367 \\
\hline
\end{tabular}




\section{Results:}

Detector cross-talk as a percentage of the total count of the standard, after subtraction of average background.

\begin{tabular}{ccccc}
\hline $\begin{array}{c}\text { Detector } \\
\text { CELL } \\
\text { No. }\end{array}$ & 1 & 2 & 3 & 4 \\
\hline $\begin{array}{c}\text { Std. in } \\
\text { CELL 1 }\end{array}$ & - & 11.2 & 4.2 & 5.6 \\
Std. in & 22.1 & - & 5.6 & 1.0 \\
CELL 2 & & & & \\
Std. in & 2.9 & 22.8 & - & 5.0 \\
CELL 3 & & & & \\
Std. in & 0.5 & 3.9 & 3.2 & - \\
CELL 4 & & & & \\
\hline
\end{tabular}




\section{(CT1) - DUTY CYCLE}

Date: April 25, 1997

Objective:

The system should demonstrate a $100 \%$ duty cycle, i.e., air collection should be continuous.

\section{Required Materials:}

State of health information and sensors window from a graphical user interface (GUI).

\section{Method/Procedure:}

GUI and determine from PNNL any functions which may prevent access to the $100 \%$ duty cycle.

\section{Observation:}

Air collection is continuous. The sample is collected on a charcoal trap for approximately $8 \mathrm{~h}$ at subambient temperatures. The collected sample is desorbed at $\sim 270^{\circ} \mathrm{C}$ while sample collection starts on a second charcoal trap that is being cooled to subambient temperatures. However, the second trap has not been completely cooled to $\sim-100^{\circ} \mathrm{C}$ from the previous desorption cycle.

Consequently, xenon is not quantitatively adsorbed for approximately 30 minutes of each sample collection cycle. The duty cycle is $94 \%$. 


\section{(CT2) - COLLECTION TIME}

Date: April 25, 1997

\section{Objective:}

The time required to collect the minimum amount of xenon should be no less than $6 \mathrm{~h}$ and no more than $24 \mathrm{~h}$.

\section{Required Materials:}

Xephyr directory; records in "xenon.log"

\section{Method/Procedure:}

Access the "xephyr" directory on the terminal window, type "showlog xenon.log" which is the data log of status changes, and press enter. Type "showlog xenon.log>ek.log" (ek can be substituted with the initials of the user) to place entries in a viewable log, and press enter. Finally, type "vedit ek.log" to view the log. Because the log only retains the latest 1000 entries, necessary information must be recorded every day before it becomes overwritten by the latest incoming data. Using the menu bar, perform a search for valves 211 (or 212,213) for Charcoal Trap A and 221 (or 222, 223) for Charcoal Trap B. Record the date and time when the valves open and close. Calculate the collection time from the open and close times.

\section{Observation:}

Collection times for 4/19/97 - 4/20/97 varied from 07:59:17 to 08:04:56 (h: min: s). Collection times between 4/21/97 - 4/24/97 stabilized and ranged from 07:56:24 to 07:56:56. PNNL added $210 \mathrm{~s}$ to the code of the system in order to achieve a collection time of approximately 08:00. The delay was added during nitrogen bleeding after the traps pressurize from elution. The change would take effect at approximately 17:30 (EST) on 4/24/97.

Because of problems with the system beginning on $4 / 25$, normal collections were not taken until 4/30. The calculated collection times for 4/30 - 5/1 ranged from 07:49:36 to 08:00:30. PNNL added $10 \mathrm{~s}$ to the code to correct for other subsequent time delays that were added later. This change would take effect at approximately $07: 00$ on 5/3/97.

\section{Results:}

May 1997 - Collection times have not been consistent.

Sept 1997 - Collection times have been consistent. 


\section{(CT3) - XENON GAS YIELD}

Date: Apr. 25, 1997

Objective:

Determine the amount of elemental xenon recovery.

Required Materials:

ARSA residual gas analyzer (RGA) and archive bottle.

Method/Procedure:

Record the RGA xenon recovery. Transfer contents of ARSA collection bottle to archive bottle. Analyze xenon content of archive bottle (BOC).

Observations:

\begin{tabular}{cccc}
\hline $\begin{array}{c}\text { Sample ID } \\
(1997)\end{array}$ & $\begin{array}{c}\text { ARSA } \\
\text { Xenon (RGA) } \\
(\mathrm{mL})\end{array}$ & $\begin{array}{c}\text { BOC } \\
\text { Xenon (Analysis) } \\
(\mathrm{mL})\end{array}$ & RGA/Anal. \\
\hline 4/21@ 1000 & 0.2 & 0.64 & 0.31 \\
$4 / 21 @ 1800$ & 0.1 & 0.62 & 0.16 \\
$4 / 22 @ 0200$ & 1.3 & 0.62 & 2.09 \\
$4 / 22 @ 1000$ & 1.6 & 0.59 & 2.71 \\
$4 / 22 @ 1800$ & 0.1 & 0.66 & 0.15 \\
$4 / 23 @ 0200$ & 3.0 & 0.30 & 10.00 \\
$4 / 23 @ 1000$ & 1.7 & $0.70^{*}$ & 2.42 \\
$4 / 30 @ 0300$ & 0.3 & 0.45 & 0.67 \\
$4 / 30 @ 1100$ & 1.8 & 0.75 & 2.40 \\
$4 / 30 @ 1900$ & 2.1 & 0.53 & 3.96 \\
$4 / 30 @ 0300$ & 0.3 & 0.45 & 0.67 \\
\hline
\end{tabular}




\begin{tabular}{cccc}
\hline $\begin{array}{c}\text { Sample ID } \\
(1997)\end{array}$ & $\begin{array}{c}\text { ARSA } \\
\text { Xenon }(\mathrm{RGA}) \\
(\mathrm{mL})\end{array}$ & $\begin{array}{c}\text { BOC } \\
\text { Xenon (Analysis) } \\
(\mathrm{mL})\end{array}$ & RGA/Anal. \\
\hline $4 / 30 @ 1100$ & 1.8 & 0.75 & 2.40 \\
$4 / 30 @ 1900$ & 2.1 & 0.53 & 3.96 \\
$5 / 01 @ 0300$ & 1.8 & 0.81 & 2.22 \\
$5 / 01 @ 1100$ & 0.6 & 0.75 & 0.80 \\
\hline
\end{tabular}

*AFTAC

See ARSA database (Table 3) for a listing of all results.

ARSA residual gas analyzer (RGA) relocated on Aug. 14. Ratio of analysis/RGA results of 92 samples collected after the relocation was $0.61 \pm 0.10$, indicating consistent results. Analysis results are consistently lower because of volume differences from the transfer of ARSA archived samples to the ACR type bottles. 


\section{(CT4) SYSTEM PURGE}

Date: May 27, 1997

Objective:

The system should completely purge all residual gas after each collection cycle.

\section{Required Materials:}

Environmental chamber, mass flow meter, compressed gas cylinder containing ${ }^{133} \mathrm{Xe}$ standard in nitrogen.

\section{Method/Procedure:}

The Environmental Chamber mixing room was not used for this experiment. All inlets and outlets of the main chamber were sealed off. Two mixing fans were placed in the chamber. ${ }^{133} \mathrm{Xe}$ was introduced into the chamber through a mass flow meter set at $100 \mathrm{~mL} / \mathrm{min}$ for a period of $45.0 \mathrm{~min}$ to bring the chamber ${ }^{133} \mathrm{Xe}$ concentration to $100 \mathrm{pCi} / \mathrm{SCM}$. Mixing continued for 30 min after the ${ }^{133} \mathrm{Xe}$ was shut off.

Operate the ARSA through $24-\mathrm{h}$ while introducing ${ }^{133} \mathrm{Xe}$ sufficient to generate a $20 \mathrm{pCi} / \mathrm{SCM}$ average intake level. For the next $24-\mathrm{h}$, feed the ARSA outside air.

Repeat the experiment with a ${ }^{133} \mathrm{Xe}$ concentration of $100 \mathrm{pCi} / \mathrm{SCM}$.

\section{Observation/Results:}


9/03/97 - ${ }^{133} \mathrm{Xe}$ chamber concentration @ $20 \mathrm{pCi} / \mathrm{m}^{3}$ (20\% of chamber air sampled)

\begin{tabular}{ccccccc}
\hline $\begin{array}{c}\text { Sample } \\
\text { Type }\end{array}$ & $\begin{array}{c}\text { Detector } \\
\text { No. }\end{array}$ & $\begin{array}{c}{ }^{133} \mathrm{Xe} \\
\left(\mathrm{pCi} / \mathrm{m}^{3}\right)^{*}\end{array}$ & $\begin{array}{c}\text { Gross } \\
\text { Count** }\end{array}$ & $\begin{array}{c}\text { Count } \\
\text { Time } \\
(\mathrm{min})\end{array}$ & $\begin{array}{c}\text { cpm } \\
{ }_{133} \mathrm{Xe}\end{array}$ & $\begin{array}{c}\% \\
\text { Retention }\end{array}$ \\
\hline $\begin{array}{c}\text { Pre-bkg } \\
{ }^{133} \mathrm{Xe}\end{array}$ & 1 & - & 971 & 944 & 1.02 & - \\
Post-bkg & 1 & 5.25 & 28574 & 960 & 29.8 & - \\
\hline Pre-bkg & 1 & - & 1954 & 944 & 2.06 & 3.6 \\
${ }^{133} \mathrm{Xe}$ & 2 & - & 959 & 944 & 1.02 & - \\
Post-bkg & 2 & 5.93 & 66567 & 960 & 69.3 & - \\
\hline Pre-bkg & 3 & - & 3900 & 944 & 4.13 & 4.6 \\
${ }^{133} \mathrm{Xe}$ & 3 & 7.85 & 56423 & 960 & 58.7 & - \\
Post-bkg & 3 & - & 3110 & 944 & 3.29 & 3.2 \\
\hline Pre-bkg & 4 & - & 2184 & 944 & 2.31 & - \\
${ }^{133} \mathrm{Xe}$ & 4 & 9.17 & 65353 & 960 & 68.1 & - \\
Post-bkg & 4 & - & 4196 & 944 & 4.45 & 3.3 \\
\hline Pre-bkg & 1 & - & 1954 & 944 & 2.06 & - \\
${ }^{133} \mathrm{Xe}$ & 1 & 8.64 & 60984 & 960 & 63.5 & - \\
\hline Post-bkg & 1 & - & 3842 & 944 & 4.07 & 3.3 \\
\hline Pre-bkg & 2 & - & 3900 & 944 & 4.13 & - \\
${ }^{133} \mathrm{Xe}$ & 2 & 8.26 & 82090 & 960 & 85.5 & - \\
Post-bkg & 2 & - & 4911 & 944 & 5.20 & 1.3 \\
\hline
\end{tabular}

*Value from laboratory analyses.

**Sum of the 0.31 and $0.81 \mathrm{keV}$ peaks. 
12/01/97 - ${ }^{133} \mathrm{Xe}$ chamber concentration @ $100 \mathrm{pCi} / \mathrm{m}^{3}$

\begin{tabular}{ccccccc}
\hline $\begin{array}{c}\text { Sample } \\
\text { Type }\end{array}$ & $\begin{array}{c}\text { Detector } \\
\text { No. }\end{array}$ & $\begin{array}{c}{ }^{133} \mathrm{Xe} \\
\left(\mathrm{pCi} / \mathrm{m}^{3}\right)^{*}\end{array}$ & $\begin{array}{c}\text { Gross } \\
\text { Count** }\end{array}$ & $\begin{array}{c}\text { Count } \\
\text { Time } \\
(\mathrm{min})\end{array}$ & $\begin{array}{c}\text { cpm } \\
{ }^{133} \mathrm{Xe}\end{array}$ & $\begin{array}{c}\% \\
\text { Retention }\end{array}$ \\
\hline $\begin{array}{c}\text { Pre-bkg } \\
{ }^{133} \mathrm{Xe}\end{array}$ & 3 & - & 261 & 943 & 0.28 & - \\
Post-bkg & 3 & 97.6 & 547613 & 960 & 570 & - \\
\hline Pre-bkg & 3 & - & 24039 & 943 & 25.4 & 4.4 \\
${ }^{133} \mathrm{Xe}$ & 4 & - & 312 & 944 & 0.33 & - \\
Post-bkg & 4 & 86.7 & 507731 & 960 & 529 & - \\
\hline Pre-bkg & 1 & - & 21872 & 944 & 23.2 & 4.3 \\
${ }^{133} \mathrm{Xe}$ & 1 & - & 311 & 944 & 0.33 & - \\
Post-bkg & 1 & - & 18466 & 944 & 19.5 & 4.7 \\
\hline
\end{tabular}

*Value from laboratory analyses.

** Sum of the 0.31 and $0.81 \mathrm{keV}$ peaks. 
Date: April 30, 1997

\section{Objective:}

The system should be capable of recording sample spectral information for each cycle.

\section{Required Materials:}

System or remote monitor and software.

\section{Method/Procedure:}

Conduct normal operation of the sampler. Determine that the system is performing the required storage of data by remotely requesting this information for previously analyzed data. Ensure that the stored data are correct.

\section{Observation:}

Spectra information is correctly stored. The transfer of spectral data files via modem was deleted from the test plan. 


\section{(DCT2) - COLLECTION INFORMATION}

Date: April 30, 1997

\section{Objective:}

The system shall record the collection and analysis start time for each sample.

\section{Required Materials:}

System or remote monitor and software.

\section{Method/Procedure:}

Conduct normal operation of the sampler. Remotely request start and stop times for previously analyzed data.

\section{Observation:}

May 1997 - Stop times of previously analyzed data (RGA, background and sample files) are obtainable as file ID. Start times are not recorded, they must be calculated.

September 1997 - Problem corrected. Start times obtained from sample.summary file. Listing contains EML sync time, detector number, ARSA system archive bottle and charcoal trap gas stream number. 


\section{(DCT3) - COMMUNICATIONS}

Date: April 30, 1997

Objective:

The data stored in the system should be provided to a serial port for communication.

Required Material:

Modems and modem communications protocol.

\section{Method/Procedure:}

Conduct normal operation of the sampler. Determine that the system is providing data via a standard RS-232 serial port by monitoring its signals.

\section{Observation:}

Present communications to EML's monitor via the Internet. Modem not provided for ARSA system. File transfer via standard RS-232 serial port deleted from the test plan. 


\section{(DCT4) -REMOTE PROGRAMMABILITY}

Date: November 1997

\section{Objective:}

Determine that the system can be remotely reprogrammed by modifying the operating instructions and inspecting the transmitted and archived data files.

\section{Required Material:}

Modem, modem communications protocol and LAN.

\section{Method/Procedure:}

Determine that the system can be remotely programmed by modifying operating instructions and inspecting the transmitted and archived data files.

\section{Observation:}

Operating instructions routinely modified from PNNL via LAN. However, communications response time extremely is slow. Modem test was deleted from the test plan. 
Date: May 30, 1997

\section{Objective:}

The unit shall record background, calibration, and sample spectral information separately for each collection/analysis cycle.

\section{Required Materials:}

MCA and RGA files from the GUI.

\section{Method/Procedure:}

Access and inspect MCA and RGA files.

\section{Observation:}

All sample spectral information, xenon recoveries (RGA files) and calibrations (stability determined from ${ }^{154,155} \mathrm{Eu}$ sources) were recorded. 


\section{(DT1) - AIRSTREAM BLOCKED}

Date: February 2, 1998

\section{Objective:}

Determine the effects from the blocked input and output airstream.

\section{Required Materials:}

None

\section{Method/Procedure:}

Test not conducted. Review "anticipated result" provided by PNNL.

\section{Observation:}

PNNL anticipated result:

"If the airstream were blocked at the input to the main compressor, the compressor would decrease the output flow and eventually become hot. The state-of-health software would recognize the decrease in output flow and pressure, as well as the increase in compressor temperature. The system will shutdown before overheating. It is anticipated that this event would not damage any part of the system."

TOD anticipated results:

"If the airstream were blocked at the input to the ARSA, from observation of the equipment during its test at EML, the state-of-health software would recognize the deficiency of the airflow either by decreased flow rates or increased temperatures and shut the system down to prevent further damage to the individual components."

"If the output airstream were blocked, the state-of-health software should be able to recognize the decrease of flow through the system or the ARSA might stay in a state waiting for a pressure to decrease before resuming sample acquisition. Either of these instances would pose significant warning signs to the software and prevent the system from continued operation and damage to itself." 


\section{(DT2) - WATER INGESTION}

Date: February 2, 1998

\section{Objective:}

Determine the effects from input ingestion of a significant amount of water (1 gallon or more).

Required Materials:

None.

\section{Method/Procedure:}

Test not conducted. Review "anticipated result" provided by PNNL.

\section{Observation:}

If the system was to "ingest" a significant amount of water, the system's dew point would probably increase rapidly, and the pressure and flow of the compressor would be affected. As in test DT1, the state-of-health would notice and shut the system down. If the water ingestion occurred at the inlet to the compressor, the compressor may become irreparably damaged and it may have to be replaced. In addition, it would be likely that the adsorbents in the drying columns (preferably the columns themselves) would need to be replaced. Before new samples could be taken, the chiller and associated tubing would have to be completely dried out."

TOD anticipated result:

"If the system was to "ingest" a significant amount of water, the compressor would most likely be damaged by the ingestion. The one column that was in the sample path at that point having no flow through it would take little damage. The belief is that the compressor would not sufficiently process the water through itself into the column. Any further damage by the water escaping from the compressor through damage or any other means would only drain out of the system, the compressor due to weight and size limits sits at the bottom of the equipment. ARSA is not required to be waterproof and would simply drain the water to the floor." 


\section{(DT3) - DRAIN CLOGS}

Date: February 2, 1998

\section{Objective:}

Determine the effects from water drain (from dryer column) clogs.

\section{Required Materials:}

None.

\section{Method/Procedure:}

Test not conducted. Review "anticipated result" provided by PNNL.

\section{Observation:}

PNNL anticipated result:

"The ARSA does not currently produce water. Instead the ARSA produces a stream of slightly humid air. If this stream were to blocked, there would be a build up of pressure at the output of the drying columns. Eventually, the regeneration flow would be affected (lowered) and the system's dew point would go bad. This would most likely not damage the system."

TOD anticipated result:

"The ARSA extracts the water in the columns through regeneration of the columns and back flowing the columns with a carrier gas $\left(\mathrm{N}_{2}\right.$ in this case). The columns themselves would not be able to effectively be regenerated if this carrier gas stream were to be blocked, the inability of the columns to remove water from the system may lead to water vapor getting into the components down the sample flow path. The dew point sensors in the ARSA should at this point be able to shut the system down before serious damage could occur to the system." 


\section{(DT4) - FLOODING}

Date: February 2, 1998

\section{Objective:}

Determine the effects if the base of the unit is flooded to 6 inches and 12 inches. Test is not actually conducted..

Required Materials:

None

Method/Procedure:

Review "anticipated result" proveded by PNNL (given in observation).

Observation:

PNNL anticipated result:

"If the room was flooded with $6-12$ inches of water, the system would most likely blow fuses and the computer would notify a technician. It is possible for very deep waters to flood the detection system, and possible severe damage could then occur to the counting system and other low-lying equipment like the turbo pump and the main compressor."

TOD anticipated result:

"If the room was flooded with 6-12 inches of water, the system hopefully would not be energized, having been powered down remotely or having local power off. If this were not possible, both equipment fuses and the main power box would very likely short out and trip the circuit breakers or blow fuses. In the event of high water levels, any equipment that is immersed could be damaged, since final component placement has not been firmly set, speculation as to which components would be fruitless." 


\section{(DT5) - VOLTAGE SPIKE}

Date: February 2, 1998

Objective:

Determine the effects from water drain (from the dryer column) clogs.

\section{Required Materials:}

None

\section{Method/Procedure:}

Test not conducted. TOD review "anticipated result" provided by PNNL (given in observation).

\section{Observation:}

"If a very large voltage spike (e.g., a lightning strike) were to happen, the uninterruptible power supply would probably be destroyed along with the computer and the rest of the electrical equipment. With the computer destroyed, all valves will revert to their unenergized state, normally closed, so that all of the pieces of the system would be isolated. It is likely that major parts of the system would need to be replaced."

TOD anticipated result:

"If a large voltage spike (e.g., a lightning strike) were to occur, any electrical parts may be damaged, destroyed, have their life expectancies shortened or start to exhibit erratic or intermittent behavior. All highly sensitive equipment would be most affected, i.e., the computer control system, counting system and any other micro processor base or micro electronic device. Assuming the main power and the computer were still available, if any parameter or any component monitored in the state-of-health software fails, the ARSA would shut down. If the main power fails, ARSA would return to its unpowered start-up state." 


\section{(OT1) - MODULAR DESIGN}

Date: March 9, 1998

\section{Objective:}

The system should use a modular design. The operational manual specifications should be sufficient to permit assembly by 2 people in 2 days.

\section{Required Materials:}

Witness installation and verify.

\section{Method/Procedure:}

The first installation procedure took 4 days to complete. On the second installation, after repairs were performed at PNNL, the installation took 2 days.

\section{Observation:}

Any technician having a working knowledge of the ARSA should be able to assemble this equipment. Modularity and access to parts of the equipment are within reason. The ideas presented during testing suggest these design concepts were kept in mind and used at all possible times, when they were cost effective and possible. The only exception to this is the valves and piping under the process deck, a crane must be on hand to remove or install the deck in a timely manor. Repair and installation times may suffer. Second installation times were within standards. 


\section{(OT2) - OPERATIONS}

Date: March 9, 1998

\section{Objective:}

EML: Note deficiencies observed in the user's manual.

TOD: Review user's manual and EML observations. Report any recommended changes in the user's manual.

\section{Required Materials:}

User's manual.

\section{Method/Procedure:}

Read manual and compare it to actual operation and equipment.

\section{Observation:}

EML: Update user's manual.

TOD: The manual needs to be brought up to date to reflect all of the changes made.

Documentation of the system was shown to me at EML, drawings and manufacturers' catalogs were on hand. This information was very complete at that time, but the configuration was still not set and any further changes will need to be included in any future documentation. 
Date: May 5, 1997

Objective:

The system shall be capable of unattended operation.

\section{Required Materials:}

- Original thermocouple, Heatcon Inc., Part \#T29006U-00-19-27024-2

- Replacement thermocouple, Heatcon Inc., Part \#T29011(1/4)U-00-19-27024-2

- "Plug" thermocouple, Heatcon Inc., Part \#T29002(5/8)U-00-19-27036-2

- Thermocouple welder, Burrel Corp., Kup-L-Weld, Cat. No.73-075, $115 \mathrm{~V}$

- Anafaze 16CLS Controller

- Calibration Gas, NorLab, Norco, Inc. ( $3 \% \mathrm{CO} 2, \mathrm{O} 2, \mathrm{~N} 2, \mathrm{Kr}, \mathrm{Xe}, \sim 85 \% \mathrm{Ar})$

- Operator's check list from User's Manual (Table 8.1)

\section{Method/Procedure:}

Routinely check that critical parameters are within critical ranges specified in the Operator's check list by viewing sensors, RGA, MCA files, state-of-health information, and by visual inspection of other indicators (gauges, lights, traps).

\section{Observation:}

4/10/97 (100) -- Two thermocouples for charcoal trap B failed. Replacements were incorrect in length.

4/11/97 (101) 0630 EST -- During the calibration with the Eu sources, the systam crashed because the software program failed to accurately locate the position of the calibration source. PNNL started counting of detector for cell 2 on 4/14/97.

4/14/97 (104) -- PNNL temporarily repaired thermocouples for charcoal trap B. The rating for these thermocouples were $<250^{\circ} \mathrm{C}$ despite the system requirement for $>250^{\circ} \mathrm{C}$. However, $<250^{\circ} \mathrm{C}$ thermocouples will continue to be used because the Swagelocks of the traps can only accommodate $1 / 8$ inch. PNNL replaced the original thermocouple connected to the controller with a "plug" and replaced the thermocouple connected to the computer with a replacement (despite its incorrect length). Thermocouple wiring was also wrapped and welded on the outside of the trap to note differences in temperature readings. Welds of cut wires were made as necessary. 
4/15/97 (105) -- PNNL repaired charcoal trap A thermocouples by switching the wiring. Due to the short length of the thermocouple leads, wire cuts and welds were necessary. The thermocouple wire that was connected to the computer was cut and welded to a thermocouple assembled and located on the outside of the trap. The thermocouple wire that was connected to the PID was then cut and welded to the original thermocouple connected to the computer. During the welding, the cable leading to the controller was not disconnected. A short circuit caused the controller to fail. Power was then turned off for everything except the detector which was running the Eu calibration. Subsequently, pressure was not sufficient to send the Eu source back to its storage position. The computer received an error message which then resulted in the loss of the sample in cell 3.

4/16/97 (106) -- PNNL installed and tuned new controller.

4/17/97 (107) -- PNNL completed calibration of the RGA by introducing calibration gas into the RGA repeatedly (at different pressures, etc.).

4/18/97 (108) -- PNNL programmed the instrument to collect samples every other $8 \mathrm{~h}$ for crosstalk determination.

4/21/97 (111) -- PNNL replaced tygon-tubing from the vacuum outlet with flexible stainless steel tubing to prevent future kinking of tube when the instrument would be pulled out for maintenance. PNNL restarted the instrument for its normal 8 hour sample collection at 10:01(EDT).

4/24/97 (114) -- PNNL increases the flow of the eluting gas through the xenon capture trap by 2-4 L/min to cause faster cooling to more effectively trap xenon after regeneration.

4/25/97 (115) -- Software problems with the RGA caused the system to shut-down. During the attempt to restart the system, PNNL updated the code with a newer Uninterruptible Power Supply version. PNNL remotely operated the system and instructed EML to shutdown the main power supply to the instrument. After unsuccessful attempts to shut the main breakers, EML was told toshut-down the computer and restart the code. Because the turbo-pump showed signs of malfunctioning, PNNL shutdown the system for the weekend.

4/28/97 (118) -- PNNL instructed EML to restart the system at 11:07; the system was remotely restarted at 18:00.

4/29/97 (119) -- The turbo pump failed; PNNL instructed EML to manually restart the turbopump. At 15:30, EML noticed an error message on the terminal. At 17:40, EML detected a gas leak at the first of six solenoid valves at the top rear of the moveable chassis. PNNL instructed EML to reinsert the associated green tube and the leak stopped; during reinsertion, high pressure gas escaped from the tube and some liquid was emitted. EML restarted the system at 18:03. 
4/30/97 (120) -- EML observed that the turbo-pump was shutdown. PNNL informs us that the safety program shut off the turbo-pump and would remotely restart the system at 11:00. At 18:15, we detect the same gas leak as that of $4 / 29$; the same measures were taken to stop the leak.

5/01/97 (121) -- The ascarite trap was almost expended. PNNL switched the system to a new trap beginning at 18:00.

5/02/97 (123) -- EML replaced the nitrogen tank (400 psig) with a new one (2650 psig).

5/03/97 (124) -- ARSA overheated and created a smoke condition. EML shutdown the system.

5/06/97 (126) -- PNNL arrived to inspect and repair the system.

5/07/97 (127) -- PNNL cleaned, dismantled, and packed the system for shipment to PNNL for repair and replacement of damaged parts. PNNL submitted a report to EML of their findings and subsequent corrective actions (see 'ARSA Malfunction at EML' by T. Bowyer et al.).

\section{7/21/97(202)--REINSTALLATION OF ARSA}

7/25/97 (206) -- Normal collection begins.

7/28/97 (209) -- Manual valve to RGA was not turned on, sample collections were normal, however, there were no reliable RGA results. Samples indicated the presence of radon.

7/29/97 (210) -- ARSA shutdown. Nitrogen flow not turned on.

7/30/97 (211) -- System shutdown due to low air flow at the start of the run.

$7 / 31 / 97$ (212) -- Safety shutdown at $0200 \mathrm{~h}$ because regeneration pressure was too low. Main valve 222 not working (no voltage when turned on).

8/01/97 (213) -- System intentionally shutdown because of building electrical shutdown.

8/11/97 (223) -- System shutdown. System restarted and shutdown. Possible thermocouple problem on dryer $\mathrm{A}$.

8/13/97 (225) -- Both dryers outside thermocouples replaced by PNNL personnel. Recalibration of RGA was attempted.

8/14/97 (226) -- RGA relocated from top of electronics cabinet to floor. Pneumatic connection to RGA was modified. 
8/18/97 (230) -- Ascarite trap Valve \#6 leaking. Switched to position \#5. Male fitting of ascarite trap is broken. The ascarite trap was replaced. Condensate water bottle was emptied. Started air sample input humidity test.

8/25/97 (237) -- PNNL requested delay of ${ }^{133}$ Xe spike test until the radon contamination in the detector cells has been reduced.

8/29/97 (241) -- Leak in archive bottle \#6. Software modified to omit bottle \#6 for storage of analysis sample. System shutdown by PNNL.

9/02/97 (245) -- Condensate trap drained.

9/10/97 (253) -- Electrical connector and gas line switched from V431 to V222.

9/11/97 (254) -- Air compressor problem. Replacement compressor shipped from PNNL.

9/12/97 (255) -- Condensate trap drained. Clippard valve failure resulted in long sample counting times. System was shutdown by PNNL.

9/15/97 (258) - Compressor replaced by PNNL personnel. Ascarite trap was replaced.

9/16/97 (259) -- Drained condensate trap. Air intake manifold was replaced and sealed with Apiezon putty and Mortite. Original manifold had open connection in rear. Sample port intake rate increased from $29 \mathrm{~L} / \mathrm{min}$ to $100 \mathrm{~L} / \mathrm{min}$.

9/17/97 (260) -- Reset overheat breaker for thermocouple. System was shutdown and restarted.

9/18/97 (261) -- Problem with charcoal trap thermocouple. System was shutdown.

9/23/97 (266) -- Detector system computer was restarted to determine the reproducibility of counting the ${ }^{154,155} \mathrm{Eu}$ secondary standards.

9/30/97 (273) -- PNNL personnel replaced over temperature thermocouples in both charcoal traps.

10/02/97 (275) -- PNNL personnel emptied and refilled the 5A molecular sieve trap with a fresh sieve. Replaced both $4.0 \mathrm{amp}$ dryer heater circuit breakers with $7.5 \mathrm{amp}$ breakers. Nitrogen carrier gas cylinder replaced.

10/06/97 (279) -- System not running. Problem with sticking valves $(211,212,221,222)$. Possible problem with dew point sensor.

10/09/97 (282) -- Solenoid valve bank repaired by PNNL personnel. 
Date: March 9, 1998

Objective:

The system should have modular components which are easily replaced or repaired. Mean-timeto-repair (MTTR) should be less than 72-h.

\section{Required Materials:}

Evaluate each component to figure out the MTTR.

\section{Method/Procedure:}

Observed configuration modifications being done while equipment operated at EML. Queried Mark Panisko, PNNL technician, about the repairs done in the equipment's past operations. Most of the equipment's components can be replaced after the error is found in less than $1 \mathrm{~h}$ if they reside outside of the process deck, some in just minutes. The process deck is in an enclosed housing on the upper most part of the frame and is harder to service. The biggest task is to open the deck up for servicing, this adds about $4 \mathrm{~h}$ to the repair time. All repairs can be checked at the end of 1 counting cycle, $8 \mathrm{~h}$ for standard collection and counting of the gas. All estimations are based on the operating model in a laboratory setting, travel time is not added and the assumption that parts are on hand is the default.

\section{Observation:}

The equipment meets the requirement for the MTTR. 


\section{(OT5) -SERVICE INTERVAL}

Date: March 9, 1998

\section{Objective:}

The system should have a service interval of at least 6-months.

\section{Required Materials:}

Master parts list of components and company information.

\section{Method/Procedure:}

Extraction of maintenance data for individual components from manufacturer's data.

\section{Observation:}

The shortest interval of maintenance is on the main compressor and is listed from the manufacture as $5,000 \mathrm{~h}$. The next is the turbo molecular pump and it has a replacement of its rubber seals at $7,000 \mathrm{~h}$ and lubrication of its parts at $14,000 \mathrm{~h}$. All sieves, charcoal $\mathrm{AI}_{2} \mathrm{O}_{3}$ and $13 \mathrm{X}$ traps, can be replaced at $1-\mathrm{y}$ intervals. All calibrations of individual equipment can be done at $1 \mathrm{y}$, as in the case of the hygrometer. At normal consumption, the $\mathrm{N}_{2}$ purge gas will last 6 months if three $1 \mathrm{~A}$ bottles are kept on hand. If the proper amount of nitrogen gas is kept on hand, the system can run for the required 6-month service interval. 


\section{(OT6) - RESTOCKING INTERVAL}

Date: March 9, 1998

\section{Objective:}

Determine the system's restocking interval.

\section{Required Materials:}

Product list.

\section{Method/Procedure:}

Inspect the system and any related field test results. Determine and report the unit restocking intervals.

\section{Observation:}

The Ascarite traps used to remove $\mathrm{CO}_{2}$ are used in multiples by the system, these can be switched remotely into place as each trap is saturated and no longer effective in removing $\mathrm{CO}_{2}$. The capacity of the Ascarite traps is designed to be greater than the maintenance period. The Ascarite trap assembly can be sent to locations prior to scheduled maintenance. All sieves, charcoal, $\mathrm{AI}_{2} \mathrm{O}_{3}$ and $13 \mathrm{X}$ traps, can be replaced at $1-\mathrm{y}$ intervals. These sieves or the system components they are contained in can also be sent prior to normal maintenance. The purge gas nitrogen is consumed at a rate of $42 \mathrm{psig/day.} \mathrm{If} \mathrm{only} \mathrm{one} \mathrm{bottle} \mathrm{at} \mathrm{a} \mathrm{time} \mathrm{is} \mathrm{on} \mathrm{hand} \mathrm{at} \mathrm{any} \mathrm{of} \mathrm{the}$ sites, it will need to be replaced every 2 months. The restocking interval will be set to the amount of nitrogen gas kept at each location, this is the lowest interval of consumed material. 


\section{(OT7) - (MTBF) Mean Time Between Failure}

Date: March 9, 1998

Objective:

MTBF should be at least 12 months.

\section{Required Materials:}

Master parts list of components and company information.

\section{Method/Procedure:}

Collect MTBF data of the critical components from manufacturers. Critical components are those parts whose failure would cause the ARSA to fail to collect a sample. Calculate the system's MTBF using the algorithm $(1 / \mathrm{MTBF})=\Sigma\left(1 / \mathrm{MTBF}_{\mathrm{i}}\right)$.

\section{Observation:}

The components used in this calculation include: the vacuum system, main compressor, chiller, valve assemblies, heaters, and pressure relief valves. The system MTBF was calculated to be 3961 hours (approximately 165 days). 


\section{(OT8) - SELF-DIAGNOSTICS}

Date: April 18, 1997

\section{Objective:}

The system should have self-diagnostics to detect operational degradation.

\section{Required Materials:}

Operating system: cnx; Photon; Graphical User Interface

\section{Method/Procedure:}

View Graphical User Interface and access $\mathrm{SOH}$.

\section{Observation:}

May 1997 - Notification of malfunctions would be desirable.

July 1997 - Log file listing all events placed in operation. Malfunctions indicated by highlighting line in color. 


\section{(OT9)-COMMERCIAL COMPONENTS}

Date: March 9, 1998

\section{Objective:}

The system should be constructed with off-the-shelf components, where possible.

\section{Required Materials:}

Documentation of all system components.

\section{Method/Procedure:}

Inspect the parts list. Report lists of components which are not off-the-shelf. Determine what components are available commercially by reviewing documentation and list all major components modified or created for the system.

\section{Observation:}

A majority of parts can be bought from commercial vendors. The final collection trap, Ascarite trap and the counting systems sample chamber were developed for their specific task by PNNL. The cryogenic valves were bought commercially and then modified by PNNL for use. PNNL is working on getting the entire counting chamber to be made commercially, and also looking to further eliminate custom created parts. 
Date: October 24, 1997

\section{Objective:}

Sample bottle pressure cannot exceed 20 psig.

\section{Required Materials:}

ARSA collection bottles, digital manometer, vacuum system.

\section{Method/Procedure:}

Evacuate digital manometer measurement system to " 0 " absolute pressure, attach the ARSA collection bottle to the system via the quick disconnect fitting, open the bottle valve and record the pressure. Measure the sample bottle pressures for five (5) collection cycles (6 cycles measured).

\section{Observation:}

\begin{tabular}{|c|c|c|c|c|}
\hline Sample ID & $\begin{array}{c}\text { Archive Bottle } \\
\text { Pos. No. }\end{array}$ & $\begin{array}{c}\text { Xe } \\
\text { Recovery } \\
\text { RGA (mL) }\end{array}$ & $\begin{array}{c}\text { Bottle } \\
\text { Pressure } \\
\text { RGA } \\
\text { (Torr) }\end{array}$ & $\begin{array}{c}\text { Measured * } \\
\text { Pressure } \\
\text { (Torr) }\end{array}$ \\
\hline 1022971441.sam_4 & 7 & 1.0 & 377.8 & 45 \\
\hline 1022972239.sam_1 & 8 & 1.2 & 280.5 & 34 \\
\hline 1023970641.sam_2 & 9 & 0.7 & 352.0 & 44 \\
\hline 1023971439.sam_3 & 10 & 1.1 & 389.2 & 56 \\
\hline 1023972242.sam_4 & 1 & 0.8 & 441.4 & 52 \\
\hline 1024970639.sam_1 & 2 & 1.3 & 291.2 & 47 \\
\hline
\end{tabular}

*Large measurement system dead volume. 


\section{(OT11) - POWER OUTAGE RECOVERY}

Date:

Objective:

The system should have a robust power outage recovery design that allows for automatic system recovery after power outages of varying lengths and that prevents loss of stored data.

\section{Required Materials:}

ARSA, uninterruptable power supply

Method/Procedure:

Demonstrate that the system "reboots" itself after power outages of $5 \mathrm{~s}, 10 \mathrm{~min}, 3 \mathrm{~h}$, and $24 \mathrm{~h}$. Determine that data are not lost after a power outage. Simulate power outage while unit is in collection, separation and counting modes (all simultaneously running).

\section{Observation:}

Counting system backed up by uninterruptable power supply (UPS). Test not performed, however, data was stored and recovered after numerous system shutdowns. 


\section{(PGT1) - SIZE}

Date: March 28, 1997

\section{Objective:}

System dimensions will not exceed 6' × 3' x 3'.

\section{Required Materials:}

Measure the system with a Stanley Powerlock II model 33-425 tape measure.

\section{Method/Procedure:}

All measurements are approximate.

Normal system size $71^{\prime \prime}$ wide $\times 803 / 4^{\prime \prime}$ high $\times 353 / 8^{\prime \prime}$ depth. When front end is in the maintenance position (pulled down) it is $863 / 8^{\prime \prime}$ deep , 51 " deep on the right side of the front end back to the electronics rack, the front end is $471 / 2$ " wide, and the electronics rack is $231 / 2$ " wide. The RGA is 13 " wide, $213 / 4^{\prime \prime}$ deep, $271 / 2^{\prime \prime}$ tall and sits on the top right side of the system.

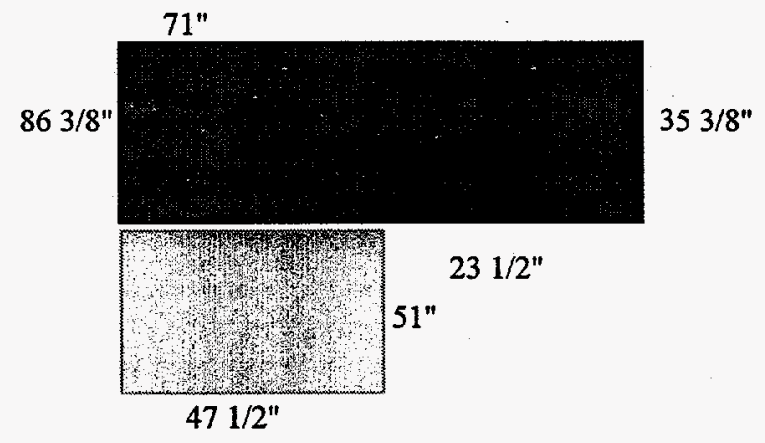

Observation: The system does not meet the size requirements. 
Date: March 9, 1998

\section{Objective:}

The system should contain modular components that do not require more than one person to lift (40 lbs), except during installation. Total system weight should be less than $1000 \mathrm{lbs}$.

\section{Required Materials:}

ARSA, scale

\section{Method/Procedure:}

Weigh the individual components.

\section{Observation:}

The front end weighs $375 \mathrm{lbs}$, the electronics rack without lead cave, archived bottles, UPS, weighs $167 \mathrm{lbs}$. The lead cave plus doors weigh $1450 \mathrm{lbs}$. The process deck weighs $370 \mathrm{lbs}$. Total system weight is recorded as $3404 \mathrm{lbs}$.

\begin{tabular}{lr}
\hline \multicolumn{1}{c}{ Component Name } & $\begin{array}{c}\text { Weight } \\
\text { (approx.) }\end{array}$ \\
\hline System enclosure & 350 \\
Main compressor & 225 \\
Air drying units & 75 \\
NaI(TI) detector & 51 \\
Scintillating gas cell assembly & \\
electronics setup & 124 \\
Lead cave assembly, doors and & 1551 \\
copper box & \\
Residual gas analyzer (RGA) & 82 \\
Computer & 33 \\
Vacuum system & 43 \\
Power failure recovery & 118 \\
Sample archive system & 15 \\
\hline
\end{tabular}

The system does not meet the weight requirements. 


\section{(PGT3) - NOISE}

Date: March 28, 1997

\section{Objective:}

The closed system should operate at $60 \mathrm{dBA}$ or less at $1 \mathrm{~m}$ from each face and corner of the unit throughout operation.

\section{Required Materials:}

Sound level meter.

\section{Method/Procedure:}

Take readings approximately $1 \mathrm{~m}$ away from the system and $1 \mathrm{~m}$ from the floor. Readings will be taken at the mid-point of each side of the A.RSA and $45^{\circ}$ off each corner.

\section{Observation:}

Two readings were taken at a range closer than 1 meter from the system because there was not sufficient room between the wall and the unit to obtain a 1 meter reading.

\begin{tabular}{lc}
\hline \multicolumn{1}{c}{ Position } & SPL (dBa) \\
\hline Front left corner & 65 \\
Left side midpoint & 67 \\
Back left corner & 70 \\
Back midpoint & $76^{*}$ \\
Back right corner & $71^{* *}$ \\
Right side midpoint & 66 \\
Front right corner & 67 \\
Front midpoint & 66 \\
\hline
\end{tabular}

${ }^{*}$ Reading taken at 20 inches

**Reading taken at 27 inches

The system does not meet the noise requirement. 


\section{(PGT4) - TEMPERATURE RANGE}

Date: February 3, 1998

\section{Objective:}

The system should be capable of operating between $50^{\circ}$ and $100^{\circ} \mathrm{F}$.

\section{Required Materials:}

Temperature, humidity recorder (Davis Weather Monitor II with Weatherlink Software).

\section{Method/Procedure:}

Record the temperature and humidity of the room at 15-min intervals. For higher temperature conditions, allow the room to reach outside summer temperature conditions.

\section{Observation:}

No environmental chamber tests are to be performed. No apparent malfunctions, damage to components or effects on the analytical functions at the room temperature range of $65^{\circ}$ to $85^{\circ}$. 
Date: March 9, 1998

\section{Objective:}

There should be no special shipping requirements for the system. There should be no special requirements for shipping or storing anticipated replacement parts and materials, e.g., tipping, hazardous material permits, and excessive weights of dimensions.

\section{Required Materials:}

Shipping receipts and system documentation.

\section{Method/Procedure:}

The system was shipped to EML by truck. The system's tubing is delicate enough to require special and careful handling during shipping so as to prevent leaks in the system. The system also contains Ascarite and small amounts of ${ }^{154} \mathrm{Eu}$ and ${ }^{155} \mathrm{Eu}$. The Ascarite is a class 8 hazardous material. It must be shipped according to Department of Transportation (DOT) 49 CFR 172.101. The Eu sources are a class 7 hazardous material. They must be shipped according to DOT 49 CFR 173.422. Both materials do not need permits to be shipped, but must meet the proper documentation as described above.

\section{Observation:}

The system meets the requirements for shipping. 
Date: August 18, 1997

\section{Objective:}

The unit should be able to operate with the intake stream at humidity between $0 \%$ and $100 \%$.

\section{Required Materials:}

Environmental Test Chamber with temperature-humidity controllers and recording systems.

\section{Method/Procedure:}

Attach the ARSA sample intake lines to the Environmental Chamber's access ports. Operate the unit through three complete cycles with the humidity of the sampling airstream held constant at $<20 \%$ and three cycles at $50 \%$. At the maximum humidity possible $(>80 \%)$ operate the system through nine cycles with PNNL personnel present for observation. Hold temperature at $75^{\circ}$ for all humidity conditions. Determine that all analytical functions perform within specifications for each of these cases by unit observation and examination of data files.

\section{Observation:}

August 18,1997 : three 8 -h sampling cycles at $20^{\circ} \mathrm{C}$ and $20 \%$ humidity.

\begin{tabular}{ccccccc}
\hline $\begin{array}{c}\text { Sample } \\
\text { Date } \\
(1997)\end{array}$ & J-Date & $\begin{array}{c}\text { Clock } \\
\text { Time }\end{array}$ & $\begin{array}{c}\text { Average } \\
\text { Temp. }\end{array}$ & $\begin{array}{c}\text { Average } \\
\% \text { Hum. }\end{array}$ & $\begin{array}{c}\text { Xe } \\
\text { Recov. } \\
\text { (mL, } \\
\text { RGA) }\end{array}$ & $\begin{array}{c}\text { Xe } \\
\text { Recov. } \\
\text { (mL, } \\
\text { Anal.) }\end{array}$ \\
\hline $8 / 18$ & 230 & 1000 & 20.3 & 23.5 & 1.70 & 1.12 \\
$8 / 18$ & 230 & 1800 & 20.3 & 20.3 & 1.90 & 1.29 \\
$8 / 19$ & 231 & 0200 & 20.4 & 20.1 & 0.10 & 0.19 \\
\hline
\end{tabular}


August 19, 1997: three 8-h sampling cycles at $20^{\circ} \mathrm{C}$ and $50 \%$ humidity.

\begin{tabular}{ccccccc}
\hline $\begin{array}{c}\text { Sample } \\
\text { Date } \\
(1997)\end{array}$ & J-Date & $\begin{array}{c}\text { Clock } \\
\text { Time }\end{array}$ & $\begin{array}{c}\text { Avg. } \\
\text { Temp. }\end{array}$ & $\begin{array}{c}\text { Avg. } \\
\text { \% Hum. }\end{array}$ & $\begin{array}{c}\text { Xe } \\
\text { Recov. } \\
\text { (mL, } \\
\text { RGA) }\end{array}$ & $\begin{array}{c}\text { Recov. } \\
\text { (mL, } \\
\text { Anal.) }\end{array}$ \\
\hline $8 / 19$ & 231 & 1000 & 20.1 & 49.2 & 1.70 & 1.20 \\
$8 / 18$ & 231 & 1800 & 20.1 & 50.4 & 1.80 & 1.13 \\
$8 / 19$ & 232 & 0200 & 20.1 & 50.5 & 0.70 & 0.29 \\
\hline
\end{tabular}

August 20,1997: Thirteen 8-h sampling cycles @20 $20^{\circ} \mathrm{C}$ and $90 \%$ humidity.*

\begin{tabular}{ccccccc}
\hline $\begin{array}{c}\text { Sample } \\
\text { Date } \\
(1997)\end{array}$ & J-Date & $\begin{array}{c}\text { Clock } \\
\text { Time }\end{array}$ & $\begin{array}{c}\text { Avg. } \\
\text { Temp. }\end{array}$ & $\begin{array}{c}\text { Avg. } \\
\text { \% Hum. }\end{array}$ & $\begin{array}{c}\text { Xecov. } \\
\text { (mL, } \\
\text { RGA) }\end{array}$ & $\begin{array}{c}\text { Xe } \\
\text { Recov. } \\
\text { (mL, } \\
\text { Anal.) }\end{array}$ \\
\hline $8 / 20$ & 232 & 1000 & 21.0 & 83.8 & 1.90 & 1.17 \\
$8 / 20$ & 232 & 1800 & 20.3 & 88.3 & 1.90 & 1.02 \\
$8 / 21$ & 233 & 0200 & 20.3 & 88.4 & 1.10 & 0.65 \\
$8 / 21$ & 233 & 1000 & 20.1 & 88.2 & 1.20 & 0.71 \\
$8 / 21$ & 233 & 1800 & 20.0 & 87.9 & 1.30 & 0.81 \\
$8 / 22$ & 234 & 0200 & 20.2 & 88.0 & 1.30 & 0.73 \\
$8 / 22$ & 234 & 1000 & 20.1 & 88.3 & 0 & 0.10 \\
$8 / 22$ & 234 & 1800 & 20.4 & 88.3 & 1.30 & 0.71 \\
$8 / 23$ & 235 & 0200 & 20.2 & 88.3 & 1.30 & 0.77 \\
$8 / 23$ & 235 & 1000 & 20.4 & 88.3 & 1.40 & 0.91 \\
$8 / 23$ & 235 & 1800 & 19.9 & 88.0 & 1.20 & 0.74 \\
$8 / 24$ & 236 & 0200 & 19.8 & 87.6 & 1.40 & 0.83 \\
\hline
\end{tabular}

* Chamber failed to revert to ambient temperature and humidity levels after nine sampling cycles. 


\section{Conclusion:}

Sample intake represented $\sim 20 \%$ of chamber air because of leak in the ARSA air intake manifold. Xenon recoveries decreased $\sim 33 \%$ at $90 \%$ humidity. No significant decrease at $50 \%$ humidity. Repeat of experiment at full sample intake flow $(100 \mathrm{~L} / \mathrm{min})$ canceled by AFTAC. 
Date: February 15, 1998

\section{Objective:}

Unit should be able to operate with environmental humidity (air surrounding the unit) between $0 \%$ and $100 \%$ at a temperature of $75^{\circ} \mathrm{F}$.

\section{Required Materials:}

a) Environmental Chamber (see observation)

b) Temperature-humidity recorder (Davis Weather Monitor II with Weatherlink Software).

\section{Method/Procedure:}

Operate the unit through 5 cycles each in the test chamber with the humidity of the surrounding air held constant at $<20 \%, 50 \%$ and $>80 \%$. Determine that all analytical functions perform within specifications by unit observation and examination of data files. Report any anomalies observed during the test.

\section{Observation:}

a) ARSA would not fit through chamber access doors. Would have required disassembly and reassembly in chamber. Test deleted from the test plan.

b) Room humidity ranged from $15 \%$ to $83 \%$ during the test period. No apparent malfunctions from humidity environment. 


\section{(PGT8) - NO LIQUID NITROGEN}

Date: March 9, 1998

Objective:

The system will not use liquid nitrogen.

Required Materials:

Inspect all system components and documentation.

Method/Procedure:

Inspect all system components and documentation.

Observation:

The system does not use liquid nitrogen. 


\section{(PGT9) - NO OZONE DEPLETERS}

Date: March 9, 1998

\section{Objective:}

The system will not use ozone-depleting substances.

Required Materials:

System documentation (MSDSs).

Method/Procedure:

Inspect all system components and documentation.

Observation:

The system contains no ozone-depleting substances. 


\section{(PGT10) - OPERATION COST}

Date: March 9, 1998

\section{Objective:}

The annual operation cost, additional material and supplies, technician time, and number of visits per year should be estimated for the system.

\section{Required Materials:}

Cost of all expendable components such as charcoal, Ascarite, and nitrogen. Cost per $\mathrm{kW} \mathrm{h}$, and other predictable support costs.

\section{Method/Procedure:}

A scenario of an operational site was established using estimated costs for power, expendable components, and technician visits tot he site location. The costs were summed together to form the total annual operation costs.

\section{Observation:}

The cost analysis is based on a technician visiting the site twice a year to perform preventative maintenance routines (PMRs) and restocking. Also, 2.1 times per year is included for unscheduled trips due to system failures (based on MTBF from test OT7). The total cost does not include component replacement cost or shipping and/or delivery cost to the site. PMRs for columns are considered to be at minimum intervals in this calculation, improvements in desigh may extend life expectancy.

\section{Power:}

The system used $5.65 \mathrm{~kW}$ h on average. Assumed a utility cost of $\$ 0.0687 \mathrm{~kW} \mathrm{~h}$, the annual power cost is:

$(\$ 0.0687 \mathrm{~kW} \mathrm{~h}) \times(5.65 \mathrm{~kW}) \times(24 \mathrm{~h} /$ day $) \times(365$ day $/$ year $)=\$ 3,400$ per year 
Consumables:

Ascarite $\quad(\$ 195.20 / 500 \mathrm{~g})(6$ columns $)-\$ 1,171 / \mathrm{PMR}$

Silica gel $\quad(\$ 21.20 / 500 \mathrm{~g})(6$ columns $)-\$ 127 / \mathrm{PMR}$

Charcoal $\quad(\$ 22.70 / \mathrm{kg})(2 \mathrm{~kg})-\$ 45 / \mathrm{PMR}$

Al2O3 (\$26.20/kg)(5 kg/column)(2 colums) - \$262/PMR

Nitrogen $\quad(\$ 0.02 / \mathrm{cu} \mathrm{ft})(301 \mathrm{cu} \mathrm{ft} /$ bottle)(3 bottles) - \$18/PMR

Total per 6 months: $\$ 1,623$ or $\$ 3,246$ annually

Travel

Assuming the technician visits the site 4.1 times a year (2 times for restocking/PMRs and 2.1 for failures) with a cost of $\$ 5,000 /$ visit the annual travel cost is $\$ 20,500$.

Total annual cost $\$ 7,146$ or about $\$ 75 /$ day. 


\section{(PGT11) - CALIBRATION SOURCE}

Date: March 9, 1998

\section{Objective:}

Internal calibration sources should be small enough that special transportation/handling requirements and site licenses are not needed to ship and operate the unit.

\section{Required Materials:}

Standard documentation, AFOSH standards, and other applicable regulations.

\section{Method/Procedure:}

Verify by using AFOSH standards and other applicable regulations to determine the proper levels and comparing those standards to the amount used by PNNL.

\section{Observation:}

The europium sources are a class 7 hazardous material. They must be shipped according to DOT $49 \mathrm{CFR} 173.421$ and 173.422 . The system calibtation sources have an activity of $5 \mathrm{nCi}$ each. Therefore, they do not need permits to be shipped. Documentation for shipment can be easily met and no special licenses are needed to ship or use the sources. Site licenses will not be required for operation according to Nuclear Regulatory Committee (NRC) 10 CFR 30.18, and 30.71 schedule $B$.

The system meets the shipping requirements. 


\section{(PGT12) - POWER}

Date: August 29, 1997

\section{Objective:}

Unit should draw less than $2.5 \mathrm{~kW}$ during continuous operation.

\section{Required Materials:}

Ohio Semitronics Model PTP-4531B-3 Power Test Panel using two (2) - 100 Amp current transformers. Readings taken every $2 \mathrm{~s}$ over an ARSA 8-h cycle. Data transferred via RS-232 and recorded on a PC.

\section{Method/Procedure:}

Measure the power draw of the unit through five complete operating cycles. Individual measurements should be taken on a time interval small enough to show all significant variations $(>10 \%)$ in power usage. Measure the power draw when unit is switched on and during, a short duration functions cause significant power draw. Plot power draw vs. time for a complete unit cycle. Measure and report peak power when unit is initially switched on and during any shortduration functions that seem likely to cause a significant power draw.

\section{Observation:}

Power draw (integrated power) as measured during five (5) complete 8-h cycles:

$\begin{array}{cc}\text { Date } & \underline{\mathrm{kW} / \mathrm{h}} \\ & \\ \text { 20Aug97 } & 5.60 \\ 21 \text { Aug97 } & 5.62 \\ 25 \text { Aug97 } & 5.76 \\ 27 \text { Aug97 } & 5.62 \\ 29 \text { Aug97 } & 5.67\end{array}$

Avg: $5.65 \pm 0.06$

Instantaneous power observed is composed of: (1) continuous square wave power at about $50 \%$ duty cycle with a minimum value of $3.5 \mathrm{~kW}$ and a maximum value of $7.5 \mathrm{~kW}$, and (2) short spikes on top of the square wave lasting 1 to $3 \mathrm{~s}$ and reaching a maximum of $20 \mathrm{~kW}$. 
Power Draw When Unit is Switched On (Oct 3, 1997)

Power usage after a cold start consists of an initial warm up period of 5-h during which $6 \mathrm{~kW}$ is drawn. During the first $1 / 2$ hour of this period, positive and negative spikes appear which may be due to ARSA internal setup tests. This is followed by a second warmup lasting about $3 / 4$ of an hour during which power consumption increases to $8 \mathrm{~kW}$. After this, normal operation begins and power consumption is as described above $(5.65 \mathrm{~kW} \mathrm{~h})$. 


\title{
APPENDIX B
}

\section{ARSA Questions Encountered During the EML Field Test}

\author{
K. H. Abel, T. W. Bowyer, P. L. Reeder, R. C. Thompson \\ March 5, 1998 \\ Pacific Northwest National Laboratory
}

\begin{abstract}
- Radon and Xenon activity retained by detectors (detector memory effect). Response: During the test during sample removal after counting a small fraction, on the order of $7 \%$, remained in the cell. Since the test we have explored options to reduce this residual level to about $4 \%$ by back-flushing with air during vacuum to help "rinse out" the gas in the cell. Also, because we are conducting a background count between each sample count, this residual background can be determined and subtracted from any subsequent sample.
\end{abstract}

- Frequent replacement of solenoid valves.

Response: There were several solenoid (Clippard) valves which quit working properly during the test period. This problem was traced back to improper handling and reassembly during their initial installation. Unfortunately, we were not aware of the non-interchangability of certain components within the valves when we first inspected and installed them. After inquiry with the manufacturer when our problems occurred, we were told of this restriction in handling and use. We did not have any problems with valves installed after we were informed of this restriction. The Clippard valves are high reliability solenoid valves. They are employed in other applications such as medical ventilators and cardiac monitoring equipment.

\section{- Frequent replacement of thermocouples.}

Response: Due to a misunderstanding we thought the original thermocouples were rated for continuous cycling between $-100^{\circ} \mathrm{C}$. and $+200^{\circ} \mathrm{C}$. After the initial thermocouple failures, we learned otherwise. Subsequently, these thermocouples were replaced, during the rebuild from May through July, and the replacement thermocouples have not failed. [We have switched to large (8) gauge, K type thermocouples]

\section{- Detector cross-talk.}

Response: The EML examination of detector cross-talk quantified a type of cross-talk observed during use of the europium check sources (external to the counting cells). Measurements at PNNL using radioxenon in the cells demonstrate that the cross-talk factor is several orders of magnitude lower than the one determined by EML. A summary is attached that describes the PNNL 
measurements. It also contains a detailed comparison with and critique of the EML analysis.

- Radon retention in the purification systems.

Response: Occasional instances of radon "breakthrough" into the counting system were related in most, if not all instances, to a malfunction in the "postradon" trap cooling loop. Specifically, the trap cooling did not switch on properly. The trap should be below $-40^{\circ} \mathrm{C}$ during elution in order to retain radon. The other occasions when radon "broke through" were caused because the post radon trap did not get heated to approximately $200^{\circ} \mathrm{C}$ as is required during the vacuum regeneration of this trap. We would also note that during the radon exposure tests the ARSA did remove elevated radon concentrations as required. We also noticed increased (x10 higher) radon levels after the return of the system to PNNL. We attribute this to the fact that we did not replace the molecular sieve traps after the fire, and hence they may have become poisoned. Although we did not have enough data to say this was the cause the radon problems late in the test, the radon levels did decrease significantly after the molecular sieve change out. It is important to note that the radon levels in the counting system did not preclude the ARSA from achieving the required detection efficiency in any case.

- Inability to view the beta-gamma spectra during acquisition without restarting the counting system.

Response: Since the data is gathered by the CPU on the control and data acquisition system, it is only a matter of writing the appropriate software to implement this function to happen automatically. During the field test the data was remotely or locally retrievable at any time from the counting system through two simple commands entered at ARSA terminal window.

- Slow response time for acquiring information and changing operating instructions for a system at a remote location.

Response: Most information was routinely transferred from EML to PNNL and was rapidly available via local network to view current status and stored data files, but the response time for viewing "real-time" status was at times quite slow. However, the lack of speed was primarily caused by the communications configuration at EML. During EML non-working hours communications response time was typically rapid and we were readily able to change operating instructions and even restart the system. As a fall back position, we were able to adequately contact and control, if desired, the ARSA with a text-based version of the system monitor even during slow network times. 
- Condensate trap must be manually drained at approximately seven day intervals.

Response: The small condensate trap was a short-term "fix" applied during the field test to a design weakness. This has been modified so draining is no longer required. Since the field test, we have run the ARSA for several months with the new setup with no need to empty the condensate trap.

- Nitrogen carrier gas consumption approximately 42 psig/day, which implies that six (6) 1A nitrogen gas cylinders/year will have to be made available at each remote location.

Response: This is true. Nitrogen will have to be supplied to the ARSA. There are several options, including $1 \mathrm{~A}$ nitrogen gas cylinders. This nitrogen may also be obtained via nitrogen generator, but we have tested ARSA only with nitrogen delivered in cylinders.

- Decrease in xenon recovery at $90 \%$ sample air humidity.

Response: While it is true that xenon yields decreased during the air humidity test, this decrease was not caused by the increase in humidity. Rather, at the time the humidity tests were conducted a blockage occurred in the cleanup process, specifically within the ascarite trap caused by an error in the mixing of the ascarite in the trap. The trap blockage significantly reduced the nitrogen flow from the normal $240 \mathrm{cc} / \mathrm{min}$. The decreased nitrogen flow and total volume over time resulted in incomplete elution of the xenon captured on the main trap. Incomplete elution resulted in lower xenon yield. It must be pointed out that while the yield did decrease, radioxenon concentrations were still determined, i.e., the ARSA still produced data on radioxenon levels in the atmosphere to the require sensitivity. Preliminary tests at PNNL with a new dryer configuration show that ascarite blockage will not be a future problem, and the new dryers seem to remove $\mathrm{CO}_{2}$ down to the fraction of a part-per-million level.

NOTE: The ascarite traps have nothing to do with the raw air, and hence do not react to high humidity levels. The ascarite trap is far downstream of the initial gas processing, and hence the ascarite traps encountered humidity much less than 1 ppm, even with $100 \%$ humidity.

\section{- Power consumption (5.65 kW) exceeds specification ( $2.5 \mathrm{~kW})$.}

Response: PNNL has been able to decrease power usage by at least $1 \mathrm{~kW}$ by using a different compressor and using heatless regenerative dryers. The developers feel that much greater reductions in power consumption, if achievable, would require radical redesign, beyond the time and budget available.

\section{- Total footprint exceeds specification.}

Response: The system is larger than the specification in order to increase ease of maintenance. 
Attachment:

\author{
Response: Crosstalk Experiments for PNNL ARSA \\ P. L. Reeder \\ Jan. 23, 1998
}

\title{
PNNL Experiments
}

We define crosstalk as those events in one sample cell which somehow cause a second sample cell to give a signal in real coincidence with a pulse in the NaI detector. The sample cells are surrounded by enough material that it is highly unlikely that a beta particle could penetrate from one sample cell to another. However, it is possible that a single gamma ray could be emitted from one sample cell, interact by Compton scattering in a second sample cell giving a "beta-gate" pulse, and then the scattered gamma interacts in the NaI detector. Another possibility is that a sample that has two or more gammas in coincidence emits one gamma into the $\mathrm{NaI}$ and the other gamma is absorbed or Compton scatters in a second sample cell. In either case a "true" coincidence event has occurred. If two or more beta cells give coincident pulses, the event is rejected by the logic router box.

The beta-gamma coincidence counting system is designed to look for gamma or X-ray peaks in the beta-gated gamma spectra. The quantity of interest is the net number of counts (area) under the peak after subtracting the continuum of events above and below the peak. We modify our definition of crosstalk to mean the number of events appearing in the net counts of a particular beta-gated gamma spectrum that are due to the net counts in the same peak in the gamma singles spectrum caused by a source in a different beta cell. Experimentally this is measured by placing a ${ }^{133} \mathrm{Xe}$ source in one of the sample cells and measuring the gamma singles and beta-gated gamma spectra for a different sample cell. The net area is calculated for the $31-\mathrm{keV}$-ray peak and the $81-\mathrm{keV}$ gamma peak in the gamma singles spectrum. The net area is also calculated for the same regions in the beta-gated gamma spectrum. The ratio of the net area in the beta-gated spectrum to the net area in the gamma singles spectrum is the fraction of the spike sample which is falsely associated with a different beta cell. For this procedure to work, it is essential that the sample cell without the spike be free of any residual activity from previous samples.

In principle, the crosstalk value should be corrected for the beta detection efficiency of the sample cell without the spike. This should be obvious if one considers the situation where the beta counting efficiency is extremely low - then very few counts will appear in the beta-gated gamma spectrum regardless of the gamma singles count rate. We therefore define crosstalk using the following expression:

$$
\mathrm{F}_{\mathrm{c}}=\mathrm{N}_{\beta \gamma} /\left(\varepsilon_{\beta} \bullet \mathrm{N}_{\gamma}\right)
$$


where $F_{c}$ is the crosstalk fraction, $N_{\beta \gamma}$ is the number of net counts in a particular peak in the beta-gated gamma spectrum, $\epsilon_{\beta}$ is the beta detection efficiency of that sample cell, and $N_{\gamma}$ is the number of net counts in the same peak in the gamma singles spectrum. Note that this expression is used for the situation where a strong source is located in a beta cell other than the one of interest.

An experiment was performed in Feb. 1997 in which a ${ }^{133}$ Xe spike sample was located in cell \#4 and the cross talk was determined for cells \#1 and \#2. Unfortunately cell \#3 (the closest cell to the spike sample) was not working during the test. The gamma singles count rate for the energy region from 0 to $400 \mathrm{keV}$ was $28 \mathrm{cps}$ and had ${ }^{133} \mathrm{Xe}$ peaks at 10 times the gamma singles background. Under these conditions, the crosstalk fraction for cells \#1 and \#2 were less than $5 \times 10^{-5}$ for both the $31-\mathrm{keV}$ peak and the $81-\mathrm{keV}$ peak. These values have not been corrected for the beta counting efficiency. However, this correction will make much less than a factor of two increase in the crosstalk fraction. In any case most of the results for crosstalk fraction are two sigma upper limits because no peak was seen in the beta-gated spectrum.

\section{EML Experiments}

The experiments at EML were performed using the ${ }^{154} \mathrm{Eu}$ and ${ }^{155} \mathrm{Eu}$ calibration sources supplied with the ARSA system. A direct comparison with the PNNL experiment can be made for the case where the calibration source was located next to cell \#4. The crosstalk fractions were reported as $5 \times 10^{-3}$ for cell $\# 1$ and $3.9 \times 10^{-2}$ for cell \#2 which are a factor of 100 to 1000 higher than the values resulting from the PNNL experiment. Even higher values for crosstalk between other cells were reported by EML.

There are two major differences in how crosstalk was defined and calculated by the two different laboratories. At EML, the entire gamma energy range from 0 to $400 \mathrm{keV}$ was included in the gamma singles and the beta-gated gamma spectra. More importantly, the kind of source and the location of the source were quite different. The ${ }^{154} \mathrm{Eu}$ source in particular has rather strong abundances of high energy gamma rays (up to $1200 \mathrm{keV}$ ). These high energy gamma rays are more likely than the Xe gamma rays to give enough recoil energy to a plastic scintillator cell during a Compton scattering interaction and thus produce a valid beta pulse in a distant cell. However, the location of the source is extremely important. With the Xe sample inside the beta scintillation cell, there is a very high efficiency for detecting the beta particles from the source. Thus, if a gamma ray travels from one beta cell and causes a pulse in a distant beta cell, that event will be rejected by the ARSA logic requirements which reject all events involving two or more beta cells. With an external Eu source, some of the beta particles are emitted away from the beta scintillation cell and some are absorbed by the cell holder. The resulting beta efficiency is very low for the Eu sources - in fact, the gamma rays from ${ }^{155} \mathrm{Eu}$ are conspicuously absent in the beta-gated gamma spectrum shown in page $8 \mathrm{~B}$ of the EML report in agreement with PNNL observations. With the Eu sources, a large fraction of the 
gamma rays are emitted without detection of the corresponding beta in the cell with the source. However, the gamma rays can travel to a distant cell and have a Compton scattering interaction giving a pulse which is not rejected by the beta logic requirements.

\section{Conclusions}

We believe that the experiment and analysis performed at PNNL is the correct approach to defining and quantifying crosstalk. Our conclusion is that a Xe spike sample would have to be 10,000 times more intense than a simultaneously counted sample before crosstalk would interfere with the results for Xe radioisotopes.

\section{References}

1. P. L. Reeder, "Xenon Counting System: Accidental Coincidences and Crosstalk," PNNL internal memo, July 11, 1997.

2. T. W. Bowyer, et al., "Xe Short Reports," PNNL 11655

3. R. J. Lagomarsino, E. Ku, and C. G. Sanderson, "Field Test of the PNNL Automated Radioxenon Sampler/Analyzer (ARSA), EML Interim Report, June 6, 1997 